\title{
A Global Survey of Technological Resources and Datasets on COVID-19
}

\author{
Manoj Muniswamaiah, Tilak Agerwala, Charles C. Tappert \\ Seidenberg School of CSIS, Pace University, White Plains, New York
}

\begin{abstract}
The application and successful utilization of technological resources in developing solutions to health, safety, and economic issues caused by COVID-19 indicate the importance of technology in curbing COVID-19. Also, the medical field has had to race against tie to develop and distribute the COVID-19 vaccine. This endeavour became successful with the vaccines created and approved in less than a year, a feat in medical history. Currently, much work is being done on data collection, where all significant factors impacting the disease are recorded. These factors include confirmed cases, death rates, vaccine rates, hospitalization data, and geographic regions affected by the pandemic. Continued research and use of technological resources are highly recommendable-the paper surveys list of packages, applications and datasets used to analyse COVID-19.
\end{abstract}

Keywords-Vaccination; hospitalization; confirmed cases; datasets; data science; COVID-19

\section{INTRODUCTION}

COVID-19 pandemic has affected the world; data is being collected by agencies, organizations, institutions, and other bodies that are keen on providing insights [391]. Data collection includes conducting case surveillance to gather data on demographics, clinical factors, epidemiologic characteristics, illness course, care, and history on exposure and contact. This data is needed to assess where, when, and who are most affected by the pandemic. The data available on COVID-19 is used by researchers in the medical field in evaluating different aspects of the virus. Statistical analysis tools estimate factors concerning the virus infectiousness obtained in growth rate and doubling time. Epidemiological models are used to group individuals based on their demographic data and apply mathematical formulas to find virus characteristics. Using machine learning, the time series prediction model is proposed to obtain the curve and forecast the epidemic's tendencies.

As a part of tackling the pandemic, different institutions, governments, organizations, and individuals have developed and adopted technological resources to manage the pandemic and support adherence to containment measures. Most of the technologies developed have utilized R programming, Python, Java, Kotlin, JavaScript, among other resources. Mobile applications were developed to help in contact tracing, notifications, and alerts to users if they interacted with a person infected. Dashboards have been used in visualizing COVID-19 cases across the world. This paper presents a survey of the technological resources and datasets been used by industry and academia to combat COVID-19.

Table I provide the name of the application; the details of the developer, institution, or academia; the summary of the application, its web link, and codebase link.

TABLE I. TECHNOLOGICAL RESOURCES SUMMARY TABLE

\begin{tabular}{|c|c|c|c|c|c|}
\hline Application & $\begin{array}{l}\text { Developer/industry/ } \\
\text { Academia/Universit } \\
\text { y details }\end{array}$ & Application summary & $\begin{array}{l}\text { Application } \\
\text { weblink }\end{array}$ & $\begin{array}{l}\text { Application code base } \\
\text { link }\end{array}$ & References \\
\hline $\begin{array}{l}\text { Coronavirus } \\
\text { tracker }\end{array}$ & $\begin{array}{l}\text { Developed by John } \\
\text { Coene. }\end{array}$ & $\begin{array}{l}\text { Coronavirus tracker is an R Shiny app that tracks } \\
\text { the spread of the coronavirus, based on three data } \\
\text { sources, including John Hopkins, Weixin, and } \\
\text { DXY Data. The app summarizes the coronavirus } \\
\text { statistics such as deaths, confirmed, recovered, } \\
\text { and suspected cases on a dashboard. }\end{array}$ & $\begin{array}{l}\text { https://www.coron } \\
\text { atracker.com/ [1] }\end{array}$ & $\begin{array}{l}\text { https://github.com/JohnCo } \\
\text { ene/coronavirus.git [2] }\end{array}$ & [1] [2] [3] \\
\hline $\begin{array}{l}\text { COVID-19 } \\
\text { Global } \\
\text { Cases }\end{array}$ & $\begin{array}{l}\text { Developed by } \\
\text { Christoph } \\
\text { Schoenenberger. }\end{array}$ & $\begin{array}{l}\text { COVID-19 Global cases are a shiny app that } \\
\text { displays the recent Covid- } 19 \text { developments via } \\
\text { key figures, plots, a map, and summary tables. }\end{array}$ & $\begin{array}{l}\frac{\text { https://chschoenen }}{\text { berger.shinyapps.i }} \\
\underline{\text { o/covid19_dashbo }} \\
\underline{\text { ard/ [4] }}\end{array}$ & $\begin{array}{l}\text { https://github.com/chschoe } \\
\text { nenberger/covid19_dashbo } \\
\text { ard [5] }\end{array}$ & $\begin{array}{l}{[3][4]} \\
{[5]}\end{array}$ \\
\hline $\begin{array}{l}\text { The 2019-20 } \\
\text { Coronavirus } \\
\text { Pandemic } \\
\text { A timeline }\end{array}$ & $\begin{array}{l}\text { Developed by Nico } \\
\text { Hahn }\end{array}$ & $\begin{array}{l}\text { Visualization of Covid-19 cases is a shiny } \\
\text { application that uses leaflet, plotly, and data from } \\
\text { Johns Hopkins University to visualize the novel } \\
\text { coronavirus outbreak and show data for the entire } \\
\text { world or particular countries. }\end{array}$ & $\begin{array}{l}\frac{\underline{\text { htps://nicohahn.s }}}{\underline{\text { hinyapps.io/covid }}} \\
\underline{\underline{19 /}[6]}\end{array}$ & $\begin{array}{l}\text { https://github.com/nicoFha } \\
\underline{\text { hn/covid_shiny [7] }}\end{array}$ & [3] [6] [7] \\
\hline
\end{tabular}




\begin{tabular}{|c|c|c|c|c|c|}
\hline $\begin{array}{l}\text { Modeling } \\
\text { COVID-19 } \\
\text { Spread vs } \\
\text { Healthcare } \\
\text { Capacity }\end{array}$ & $\begin{array}{l}\text { Developed by Dr. } \\
\text { Alison Hill from } \\
\text { Johns Hopkins } \\
\text { University }\end{array}$ & $\begin{array}{l}\text { This application utilizes the epidemiological } \\
\text { model based on the classic SEIR model to define } \\
\text { the Covid-19 spread and clinical progression. } \\
\text { The application provides different infection } \\
\text { trajectories, clinical interventions to curb } \\
\text { transmission, and a comparison to the current } \\
\text { healthcare capacity. }\end{array}$ & $\begin{array}{l}\frac{\text { https://alhill.shiny }}{\text { apps.io/COVID19 }} \\
\underline{\text { seir/ [8] }}\end{array}$ & $\underline{\underline{\text { https://github.com/alsnhll/ }}}$ & [3] [8] [9] \\
\hline $\begin{array}{l}\text { COVID-19 } \\
\text { Data } \\
\text { Visualizatio } \\
\text { n Platform }\end{array}$ & $\begin{array}{l}\text { Developed by } \\
\text { Shubhram Pandey. }\end{array}$ & $\begin{array}{l}\text { This is a shiny app that provides an elaborate } \\
\text { visualization of the impact of Covid-19 across } \\
\text { the globe. The application also applies natural } \\
\text { language processing from Twitter to provide } \\
\text { sentiment analysis. }\end{array}$ & $\begin{array}{l}\frac{\text { https://shubhramp }}{\text { andey.shinyapps.i }} \\
\frac{\text { o/coronaVirusViz/ }}{[10]}\end{array}$ & $\begin{array}{l}\text { https://github.com/shubhra } \\
\underline{\text { mpandey/coronaVirus- }} \\
\underline{\text { dataViz [11] }}\end{array}$ & {$[3][10][11]$} \\
\hline $\begin{array}{l}\text { Coronavirus } \\
\text { 10-day } \\
\text { forecast }\end{array}$ & $\begin{array}{l}\text { This application was } \\
\text { developed by Spatial } \\
\text { Ecology and } \\
\text { Evolution Lab } \\
\text { (SpEEL) from the } \\
\text { University of } \\
\text { Melbourne. }\end{array}$ & $\begin{array}{l}\text { It is a shiny app that provides a ten-day forecast } \\
\text { of likely coronavirus cases by country, giving } \\
\text { individuals a sense of how the Covid-19 is } \\
\text { spreading or progressing. }\end{array}$ & $\begin{array}{l}\text { https://covid19for } \\
\text { ecast.science.unim } \\
\text { elb.edu.au/_[12] }\end{array}$ & $\begin{array}{l}\text { https://github.com/benflips } \\
\text { /nCovForecast [13] }\end{array}$ & [3] [12][13] \\
\hline $\begin{array}{l}\text { Coronavirus } \\
\text { (COVID-19) } \\
\text { across the } \\
\text { world }\end{array}$ & $\begin{array}{l}\text { This application was } \\
\text { developed by Anisa } \\
\text { Dhana. }\end{array}$ & $\begin{array}{l}\text { It is a shiny app that uses a map visualization of } \\
\text { cases confirmed to monitor the spread of Covid- } \\
19 \text { across the world and graphs to visualize the } \\
\text { growth of the disease. }\end{array}$ & $\begin{array}{l}\underline{\text { https://dash.datasc }} \\
\underline{\text { ienceplus.com/cov }} \\
\underline{\text { id19/ [14] }}\end{array}$ & $\begin{array}{l}\text { https://github.com/CSSEGI } \\
\underline{\text { SandData/COVID-19 [15] }}\end{array}$ & {$[16][14]$ [15] } \\
\hline $\begin{array}{l}\text { COVID-19 } \\
\text { outbreak }\end{array}$ & $\begin{array}{l}\text { Dr. Thibaut Fabacher } \\
\text { developed this } \\
\text { application in } \\
\text { collaboration with } \\
\text { the department of } \\
\text { Public Health of the } \\
\text { Strasbourg } \\
\text { University Hospital } \\
\text { and the Laboratory of } \\
\text { Biostatistics and } \\
\text { Medical Informatics } \\
\text { of the Strasbourg } \\
\text { Medicine Faculty. }\end{array}$ & $\begin{array}{l}\text { The application displays an interactive map that } \\
\text { indicates the worldwide monitoring of Covid-19 } \\
\text { infection. The main area of focus of the app is on } \\
\text { the evolution of the number of Covid-19 cases } \\
\text { per country for a given period. }\end{array}$ & $\begin{array}{l}\text { https://thibautfaba } \\
\text { cher.shinyapps.io/ } \\
\text { covid-19/ [17] }\end{array}$ & $\begin{array}{l}\text { https://github.com/DrFabac } \\
\underline{\text { h/Corona [18] }}\end{array}$ & [3] [17] [18] \\
\hline $\begin{array}{l}\text { Corona } \\
\text { trajectories }\end{array}$ & $\begin{array}{l}\text { This application was } \\
\text { developed by André } \\
\text { Calero Valdez, from } \\
\text { RWTH Aachen } \\
\text { University. }\end{array}$ & $\begin{array}{l}\text { The application uses two graphs to compare the } \\
\text { number of confirmed cases and the deaths from } \\
\text { Covid-19 with the country's trajectories. The } \\
\text { application also allows users to compare the case } \\
\text { number and growth rate of the Covid-19 } \\
\text { pandemic per country using a table. }\end{array}$ & $\begin{array}{l}\text { https://andrecalero } \\
\text { valdez.shinyapps.i } \\
\underline{\text { o/CovidTimeSerie }} \\
\underline{\text { sTest/ [19] }}\end{array}$ & $\begin{array}{l}\text { https://github.com/Sumidu/ } \\
\text { covid19shiny [20] }\end{array}$ & [3] [19] [20] \\
\hline $\begin{array}{l}\text { Flatten the } \\
\text { curve }\end{array}$ & $\begin{array}{l}\text { Tinu Schneider } \\
\text { developed }\end{array}$ & $\begin{array}{l}\text { this application. } \\
\text { In an interactive way, the app illustrates the } \\
\text { different scenarios behind the \#FlattenTheCurve } \\
\text { message. }\end{array}$ & $\begin{array}{l}\frac{\text { https://tinu.shinya }}{\text { pps.io/Flatten the }} \\
\text { _Curve/ [21] }\end{array}$ & $\begin{array}{l}\text { https://github.com/tinu- } \\
\text { schneider/Flatten the_Cur } \\
\text { ve [22] }\end{array}$ & [3] [21] [22] \\
\hline $\begin{array}{l}\text { Explore the } \\
\text { Spread of } \\
\text { Covid-19 }\end{array}$ & $\begin{array}{l}\text { Joachim Gassen } \\
\text { developed this } \\
\text { application, }\end{array}$ & $\begin{array}{l}\text { The application allows users to visualize } \\
\text { confirmed, recovered cases and reported deaths } \\
\text { for several countries via one summary graph. }\end{array}$ & $\begin{array}{l}\frac{\text { https://jgassen.shi }}{\text { nyapps.io/tidycovi }} \\
\underline{\underline{\mathrm{d} 19 /}[23]}\end{array}$ & $\begin{array}{l}\text { https://statsandr.com/blog/t } \\
\text { op-r-resources-on-covid- } \\
\underline{\text { 19- }} \\
\text { coronavirus/\#tidycovid19 } \\
{[24]}\end{array}$ & [3] [23] [24] \\
\hline COVID-19 & $\begin{array}{l}\text { Sebastian Engel- } \\
\text { Wolf developed the } \\
\text { application }\end{array}$ & $\begin{array}{l}\text { The application visualizes elegantly collected } \\
\text { Covid-19 data, including the confirmed cases, } \\
\text { Maximum time of exponential growth in a row, } \\
\text { deaths, populations, and Confirmed cases on } \\
\text { 100,000 inhabitants, exponential growth, and the } \\
\text { population. }\end{array}$ & $\begin{array}{l}\frac{\text { https://sebastianw }}{\text { olf.shinyapps.io/C }} \\
\underline{\text { orona-Shiny/ [25] }}\end{array}$ & $\begin{array}{l}\text { https://github.com/zapping } \\
\text { seb/coronashiny [26] }\end{array}$ & [3] [25] [26] \\
\hline $\begin{array}{l}\text { Simulation } \\
\text { tool. } \\
\text { COVID-19 } \\
\text { epidemic in } \\
\text { Togo - West } \\
\text { Africa }\end{array}$ & $\begin{array}{l}\text { Dr. Kankoé Sallah } \\
\text { developed this } \\
\text { application. }\end{array}$ & $\begin{array}{l}\text { Uses SEIR metapopulation model with mobility } \\
\text { between catchment areas to describe the country- } \\
\text { level spread of COVID-19 and the impact of } \\
\text { interventions in Togo, West Africa. }\end{array}$ & $\begin{array}{l}\text { https://c2m- } \\
\text { africa.shinyapps.i } \\
\underline{\text { o/togo-covid- }} \\
\underline{\text { shiny/ [27] }}\end{array}$ & & [3] [27] \\
\hline Animating & Nathan Chaney & This application indicates a map animation of & & https://www.nathanchaney. & [30] [29] \\
\hline
\end{tabular}




\begin{tabular}{|c|c|c|c|c|c|}
\hline $\begin{array}{l}\text { COVID-19 } \\
\text { hotspots } \\
\text { over time }\end{array}$ & $\begin{array}{l}\text { developed this } \\
\text { application }\end{array}$ & $\begin{array}{l}\text { new Covid-19 cases in the U.S.A measured in a } \\
\text { 7-day rolling average. }\end{array}$ & & $\mathrm{com} /[29]$ & \\
\hline $\begin{array}{l}\text { Covid-19- } \\
\text { prediction }\end{array}$ & $\begin{array}{l}\text { This application was } \\
\text { developed by Manuel } \\
\text { Oviedo and Manuel } \\
\text { Febrero of Modestya } \\
\text { research group of the } \\
\text { University of } \\
\text { Santiago de } \\
\text { Compostela. }\end{array}$ & $\begin{array}{l}\text { The application is a shiny app that provides a 5- } \\
\text { day horizon prediction growth rate of Covid-19 } \\
\text { using the evolution during the past 15-day } \\
\text { growth rate. The prediction is performed using } \\
\text { three functional regression models fitted and } \\
\text { estimated on available data. Apart from the } \\
\text { prediction values, the app provides an interactive } \\
\text { table and plot for the expected number of } \\
\text { accumulated cases and new daily confirmed and } \\
\text { death cases. }\end{array}$ & $\begin{array}{l}\text { http://modestya.us } \\
\text { c.es:3838/covid19 } \\
\text { prediction/ [33] }\end{array}$ & $\frac{\text { https://github.com/arnimpd }}{\text { m/Covid-19-prediction }}$ & [3] [33] [34] \\
\hline $\begin{array}{l}\text { Healthcare } \\
\text { worker } \\
\text { deaths from } \\
\text { novel } \\
\text { Coronavirus } \\
\text { (COVID-19) } \\
\text { in the US }\end{array}$ & $\begin{array}{l}\text { Jonathan Gross } \\
\text { developed this } \\
\text { application }\end{array}$ & $\begin{array}{l}\text { The application is a shiny app that visualizes the } \\
\text { U.S. health workers' deaths from Covid-19 } \\
\text { reported on media outlets or news. The } \\
\text { application is developed using R code with a map } \\
\text { on the main page using Leaflet with tabs for } \\
\text { additional graphs, including time series, } \\
\text { histograms, and bar charts. }\end{array}$ & $\begin{array}{l}\underline{\text { https://jontheepi.s }} \\
\underline{\text { hinyapps.io/hcwc }} \\
\underline{\text { oronavirus/ [35] }}\end{array}$ & $\begin{array}{l}\text { https://github.com/jontheep } \\
\underline{\text { i/hcwcoronavirus [36] }}\end{array}$ & [3] [35] [36] \\
\hline $\begin{array}{l}\text { Covid-19 } \\
\text { Hospitalizati } \\
\text { ons in } \\
\text { Belgium }\end{array}$ & $\begin{array}{l}\text { Jean-Michel Bodart } \\
\text { developed this } \\
\text { application }\end{array}$ & $\begin{array}{l}\text { The dashboard indicates the hospitalizations } \\
\text { related to Covid-19 in Belgium by province and } \\
\text { region. }\end{array}$ & $\frac{\underline{\text { htps://rpubs.com/ }}}{\text { JMBodart/Covid1 }}$ & $\begin{array}{l}\text { https://github.com/jmbo11 } \\
\underline{\text { 90/Covid19 [38] }}\end{array}$ & [3] [37] [38] \\
\hline $\begin{array}{l}\text { Covidminde } \\
\text { r }\end{array}$ & $\begin{array}{l}\text { This shiny app was } \\
\text { developed by the } \\
\text { Rensselaer Institute } \\
\text { for Data Exploration } \\
\text { and Applications }\end{array}$ & $\begin{array}{l}\text { The application indicates the regional differences } \\
\text { in determinants, medications, and outcome of the } \\
\text { Covid-19 pandemic across the United but with a } \\
\text { specific focus on New York. }\end{array}$ & $\frac{\text { https://covidminde }}{\underline{\text { r.idea.rpi.edu/ [39] }}}$ & $\begin{array}{l}\text { https://github.com/TheRen } \\
\text { sselaerIDEA/COVIDMIN } \\
\underline{\text { DER [40] }}\end{array}$ & [41] [39] [40] \\
\hline $\begin{array}{l}\text { COVID-19 } \\
\text { Canada Data } \\
\text { Explorer }\end{array}$ & $\begin{array}{l}\text { Petr Baranovskiy } \\
\text { developed this } \\
\text { application. }\end{array}$ & $\begin{array}{l}\text { The application is a shiny app that analyses the } \\
\text { official covid- } 19 \text { dataset from the government of } \\
\text { Canada and outputs the several indicators } \\
\text { associated with the Covid- } 19 \text { pandemic in the } \\
\text { country. }\end{array}$ & $\begin{array}{l}\underline{\text { https://dataenthusi }} \\
\text { ast.ca/apps/covid__ } \\
\underline{\text { ca/ [42] }}\end{array}$ & $\begin{array}{l}\text { https://milano- } \\
\text { r.github.io/erum2020- } \\
\text { covidr-contest/petr- } \\
\text { baranovskiy-covid-ca-data- } \\
\text { explorer.html [43] }\end{array}$ & [3] [42][43] \\
\hline $\begin{array}{l}\text { P A G T A } \\
\text { G N A: } \\
\text { Philippine } \\
\text { COVID-19 } \\
\text { Case } \\
\text { Forecasting } \\
\text { Web } \\
\text { Application }\end{array}$ & $\begin{array}{l}\text { This application was } \\
\text { developed by Jamal } \\
\text { Kay Rogers and } \\
\text { Yvonne Grace } \\
\text { Arandela. }\end{array}$ & $\begin{array}{l}\text { It is a shiny app that provides a 5-day forecast of } \\
\text { Covid-19 cases in the Philipines include the } \\
\text { confirmed new cases of infections, confirmed } \\
\text { deaths, and recovery rate. Apart from } \\
\text { forecasting, the application utilizes plots to } \\
\text { visualize the disease's ten-day forecasts and the } \\
\text { accumulated and confirmed data. The data used } \\
\text { in this application is obtained from the Johns } \\
\text { Hopkins University Center for Systems Science } \\
\text { and Engineering (JHU CSSE). }\end{array}$ & $\begin{array}{l}\frac{\text { https://jamalrogers }}{\text { app.shinyapps.io/t }} \\
\underline{\text { sforecast/ [44] }}\end{array}$ & $\frac{\text { https://github.com/fsmosca }}{\text { /COVID-19-PH-dataset }}$ & [3] [44] [45] \\
\hline $\begin{array}{l}\text { COVID-19 } \\
\text { Case \& } \\
\text { Death } \\
\text { Report } \\
\text { Number } \\
\text { Corrector }\end{array}$ & $\begin{array}{l}\text { Matt Maciejewski } \\
\text { developed this } \\
\text { application. }\end{array}$ & $\begin{array}{l}\text { This shiny application is developed and aligned } \\
\text { to make corrections of underreported Covid-19 } \\
\text { cases and death. The application applies a } \\
\text { multiplicative estimator for total deaths and cases } \\
\text { regarding the base country to perform this role. }\end{array}$ & $\begin{array}{l}\frac{\text { https://pharmhax.s }}{\text { hinyapps.io/covid- }} \\
\frac{\text { corrector-shiny/ }}{[46]}\end{array}$ & $\underline{\underline{\text { https://github.com/pharmh }}}$ & [48] [46] [47] \\
\hline $\begin{array}{l}\text { COVID19 } \\
\text { forecast }\end{array}$ & $\begin{array}{l}\text { Carlos Catania } \\
\text { developed this } \\
\text { application }\end{array}$ & $\begin{array}{l}\text { This application applies the SEIR model to } \\
\text { forecast the spread of Covid } 19 \text { in various } \\
\text { European and South American countries. }\end{array}$ & $\begin{array}{l}\text { https://harpomaxx } \\
\underline{\text { shinyapps.io/covi }} \\
\underline{\text { d19/ [49] }}\end{array}$ & $\begin{array}{l}\text { https://github.com/harpom } \\
\underline{\text { axx/COVID19 [50] }}\end{array}$ & [3] [49] [50] \\
\hline $\begin{array}{l}\text { Trafford } \\
\text { Covid-19 } \\
\text { monitor }\end{array}$ & $\begin{array}{l}\text { This is a shiny } \\
\text { application } \\
\text { developed by } \\
\text { Trafford Data Lab }\end{array}$ & $\begin{array}{l}\text { The application provides trends in confirmed } \\
\text { coronavirus cases in Trafford. }\end{array}$ & $\frac{\frac{\text { https://trafforddata }}{\text { lab.shinyapps.io/tr }}}{\frac{\text { afford covid-19/ }}{[54]}}$ & $\frac{\text { https://github.com/trafford }}{\text { DataLab/trafford_covid-19 }}$ & [53] [54] [55] \\
\hline $\begin{array}{l}\text { Covid-19 } \\
\text { Bulletin } \\
\text { Board }\end{array}$ & $\begin{array}{l}\text { Wei Su developed } \\
\text { this application }\end{array}$ & $\begin{array}{l}\text { The dashboard indicates the real-time Covid-19 } \\
\text { visualization of the various covid-19 indicators in } \\
\text { Japan, including the confirmed cases, hospital } \\
\text { discharge and deaths, positive confirmed, and } \\
\text { PCR test. }\end{array}$ & $\begin{array}{l}\text { https://covid- } \\
\underline{\text { 2019.live/en/ }}[56]\end{array}$ & $\frac{\text { https://github.com/swsoyee }}{\underline{\text { /2019-ncov-japan [57] }}}$ & [3] [56] [57] \\
\hline $\begin{array}{l}\text { Covid-19 } \\
\text { Statistics }\end{array}$ & $\begin{array}{l}\text { Carl Sansfaçon } \\
\text { developed this }\end{array}$ & $\begin{array}{l}\text { It is a WordPress plugin that applies the R } \\
\text { \{ggplot2\} graphics with ARIMA forecast and }\end{array}$ & $\begin{array}{l}\text { http://moduloinfo. } \\
\text { ca/wordpress/ [58] }\end{array}$ & $\begin{array}{l}\text { https://plugins.trac.wordpre } \\
\text { ss.org/browser/covid-19- }\end{array}$ & [3] [58] [59] \\
\hline
\end{tabular}




\begin{tabular}{|c|c|c|c|c|c|}
\hline Displayer & application & $\begin{array}{l}\text { PHP coding to display or visualize the confirmed } \\
\text { new cases of Covid-19 infection, deaths, and } \\
\text { recovered cases in various countries. The data } \\
\text { used in this application is sourced from the } \\
\text { COVID-19 Data Repository by the Centre for } \\
\text { Systems Science and Engineering (CSSE) at } \\
\text { Johns Hopkins University. }\end{array}$ & & statistics-displayer/ [59] & \\
\hline $\begin{array}{l}\text { CoronaMap } \\
\text { per }\end{array}$ & $\begin{array}{l}\text { This application was } \\
\text { developed by Peter } \\
\text { Gruber and Paolo } \\
\text { Montemurro } \\
\text { supported by } \\
\text { OxyLabs. }\end{array}$ & $\begin{array}{l}\text { The application visualizes the four-day average } \\
\text { growth indicator of Covid-19 to indicate how the } \\
\text { disease evolves after filtering out the noise. The } \\
\text { visualizations are both interactive and intuitive. }\end{array}$ & $\begin{array}{l}\text { http://coronamapp } \\
\text { er.com/ [60] }\end{array}$ & $\begin{array}{l}\text { https://github.com/JayWels } \\
\underline{\text { h/coronamap [61] }}\end{array}$ & [3] [60] [61] \\
\hline CoronaDash & $\begin{array}{l}\text { This is a shiny app } \\
\text { developed by Peter } \\
\text { Laurinec. }\end{array}$ & $\begin{array}{l}\text { This application applies visualization and data } \\
\text { mining techniques in R to compare Covid-19 } \\
\text { statistics for different countries. The Covid-19 } \\
\text { statistics displayed are obtained by using } \\
\text { exponential smoothing model to extrapolate total } \\
\text { confirmed cases; creating death trajectories; } \\
\text { using dendrogram and table of clusters averages } \\
\text { to create a multidimensional clustering; } \\
\text { developing aggregated views of the entire world; } \\
\text { and applying hierarchical clustering to compare } \\
\text { the Covid-19 case between countries. }\end{array}$ & $\begin{array}{l}\text { https://petolau.shi } \\
\underline{\text { nyapps.io/coronad }} \\
\text { ash/ [62] }\end{array}$ & $\begin{array}{l}\text { https://github.com/PetoLau } \\
\text { /CoronaDash [63] }\end{array}$ & [3] [62] [63] \\
\hline Covidfrance & $\begin{array}{l}\text { This is a shiny app } \\
\text { developed by } \\
\text { Guillaume Pressiat }\end{array}$ & $\begin{array}{l}\text { The application indicates the changes in the } \\
\text { number of Covid- } 19 \text { deaths and recoveries, } \\
\text { hospitalization, and intensive care units by the } \\
\text { department in France }\end{array}$ & $\begin{array}{l}\text { https://guillaumep } \\
\text { ressiat.shinyapps.i } \\
\text { o/covidfrance/ } \\
\text { [64] }\end{array}$ & $\begin{array}{l}\frac{\text { https://gist.github.com/Guil }}{\text { laumePressiat/0e3658624e }} \\
\frac{\text { 42f763e3e6a67df92bc6c5 }}{[65]}\end{array}$ & [3] [64] [65] \\
\hline $\begin{array}{l}\text { COVID-19 } \\
\text { Tracker }\end{array}$ & $\begin{array}{l}\text { Dr Magda Bucholc } \\
\text { developed this } \\
\text { application from } \\
\text { Ulster University }\end{array}$ & $\begin{array}{l}\text { The application reports the number of reported } \\
\text { Covid-19 cases at the local government district in } \\
\text { Northern Ireland and county level across Ireland } \\
\text { based on gender and growth rate. }\end{array}$ & $\begin{array}{l}\text { https://nicovidtrac } \\
\underline{\text { ker.org/ [66] }}\end{array}$ & $\begin{array}{l}\frac{\text { https://github.com/YouGov }}{\text {-Data/covid-19-tracker }} \\
\text { [67] }\end{array}$ & [68] [66] [67] \\
\hline $\begin{array}{l}\text { WHO } \\
\text { COVID-19 } \\
\text { Explorer }\end{array}$ & $\begin{array}{l}\text { This application was } \\
\text { developed by the } \\
\text { World Health } \\
\text { Organization (WHO) }\end{array}$ & $\begin{array}{l}\text { This application provides timely updated data } \\
\text { visualizations of Covid- } 19 \text { cases, including } \\
\text { confirmed cases and deaths by region and } \\
\text { country. }\end{array}$ & $\begin{array}{l}\frac{\text { https://worldhealt }}{\text { horg.shinyapps.io/ }} \\
\underline{\text { covid [71]_ }}\end{array}$ & $\underline{\text { https://github.com/WorldH }}$ & [3] [71] [72] \\
\hline $\begin{array}{l}\text { COVID-19 } \\
\text { Scenario } \\
\text { Analysis } \\
\text { Tool }\end{array}$ & $\begin{array}{l}\text { The MRC Centre } \\
\text { developed this } \\
\text { application for } \\
\text { Global Infectious } \\
\text { Disease Analysis } \\
\text { from the Imperial } \\
\text { College London. }\end{array}$ & $\begin{array}{l}\text { This application applies the squire R package to } \\
\text { illustrate the Covid-19 pandemic trajectories, R_t } \\
\text { \& R_eff measures, and healthcare demand for } \\
\text { different countries over time. }\end{array}$ & $\frac{\text { https://www.covid }}{\underline{\text { sim.org/v6.20210 }}}$ & $\begin{array}{l}\text { https://github.com/mrc- } \\
\text { ide/squire [74] }\end{array}$ & [3] [73] [74] \\
\hline $\begin{array}{l}\text { Coronavirus } \\
\text { Package }\end{array}$ & $\begin{array}{l}\text { Rami Krispin } \\
\text { developed this R } \\
\text { package }\end{array}$ & $\begin{array}{l}\text { This package provides a clean dataset of the } \\
\text { Covid-19 pandemic and analytics, including the } \\
\text { daily summary of the pandemic cases by state. } \\
\text { The dataset is collected from the John Hopkins } \\
\text { database. }\end{array}$ & & $\begin{array}{l}\text { https://ramikrispin.github.i } \\
\underline{\text { o/coronavirus/ [75] }}\end{array}$ & [3] [75] \\
\hline $\begin{array}{l}\text { District } \\
\text { Health } \\
\text { Information } \\
\text { Software } \\
\text { (DHIS2) }\end{array}$ & $\begin{array}{l}\text { The University of } \\
\text { Oslo developed this } \\
\text { application }\end{array}$ & $\begin{array}{l}\text { The District Health Information Software has } \\
\text { specific digital packages for Covid-19 that } \\
\text { support the pandemic's surveillance and response } \\
\text { activities. }\end{array}$ & $\begin{array}{l}\text { https://www.dhis2 } \\
\text {.org/ [76] }\end{array}$ & $\begin{array}{l}\text { https://github.com/dhis2/dh } \\
\underline{\text { is2-covid19-doc [79] }}\end{array}$ & $\begin{array}{l}{[80][76][77]} \\
{[78][79]}\end{array}$ \\
\hline $\begin{array}{l}\text { Surveillance } \\
\text {, Outbreak } \\
\text { Response } \\
\text { Managemen } \\
\text { t and } \\
\text { Analysis } \\
\text { System } \\
\text { (SORMAS) }\end{array}$ & $\begin{array}{l}\text { Helmholtz Centre for } \\
\text { Infection Research } \\
\text { developed this } \\
\text { system. }\end{array}$ & $\begin{array}{l}\text { The system performs the Covid-19 specific } \\
\text { functions that are classified into aggregates and } \\
\text { case-based functions. The aggregate functions } \\
\text { include line listing, import, and export of data in } \\
\text { CSV format; standard reporting of covid-19 } \\
\text { cases including confirmed cases, deaths, and } \\
\text { recoveries; and statistical analysis based on the } \\
\text { reports provided by charts, maps, and graphs. } \\
\text { The case-based functions include contact tracing, } \\
\text { laboratory sample management, port of entry } \\
\text { reporting, vaccination campaign, follow-up visit, } \\
\text { and enrolling and tracing patients. }\end{array}$ & $\underline{\text { https://sormas.org/ }}$ & $\begin{array}{l}\text { https://github.com/hzi- } \\
\text { braunschweig/SORMAS- } \\
\text { Project [82] }\end{array}$ & {$[80][81][82]$} \\
\hline
\end{tabular}




\begin{tabular}{|c|c|c|c|c|c|}
\hline Go.Data & $\begin{array}{l}\text { This application was } \\
\text { developed by WHO } \\
\text { in collaboration with } \\
\text { partners in the Global } \\
\text { Outbreak Alert and } \\
\text { Response Network } \\
\text { (GOARN). }\end{array}$ & $\begin{array}{l}\text { Since the outbreak of Covid-19 began, metadata } \\
\text { packages have been prepared that match the most } \\
\text { recent WHO Surveillance Guidance, including } \\
\text { uniformity with all core metadata gathered as } \\
\text { part of WHO Case Reporting Forms transmitted } \\
\text { to COVID-MART / X-MART on a daily and } \\
\text { weekly basis. If requested, this allows for } \\
\text { streamlined IDSR reporting for countries. Other } \\
\text { expanded metadata packages, such as the } \\
\text { COVID First Few Hundred Cases (FFX) } \\
\text { Protocol and the Unity Studies for HealthCare } \\
\text { Workers, are available for countries conducting } \\
\text { more extensive data collection or research } \\
\text { inquiries. }\end{array}$ & $\frac{\text { https://www.who.i }}{\text { nt/godata [83] }}$ & $\begin{array}{l}\text { https://github.com/godata- } \\
\text { who/godata [84] }\end{array}$ & [80] [83] [84] \\
\hline Epi Info & $\begin{array}{l}\text { This application was } \\
\text { developed by Centres } \\
\text { for Disease Control } \\
\text { and Prevention } \\
\text { (CDC) }\end{array}$ & $\begin{array}{l}\text { The Covid-19 specific functions include the } \\
\text { development of COVID-19 Case Surveillance } \\
\text { Forms that are customized for country, region, } \\
\text { and local requirements. Epi Info is also applied } \\
\text { in Covid-19 outbreak investigations, the } \\
\text { development of small to mid-sized disease } \\
\text { surveillance systems, the analysis, visualization, } \\
\text { and reporting (AVR) components of larger } \\
\text { systems, and continuing education in } \\
\text { epidemiology and public health analytic methods } \\
\text { at public health schools around the world. }\end{array}$ & $\begin{array}{l}\text { https://www.cdc.g } \\
\frac{\text { ov/epiinfo/support }}{\text { /downloads.html }} \\
\text { [85] }\end{array}$ & $\begin{array}{l}\text { https://github.com/Epi- } \\
\text { Info/Epi-Info-Community- } \\
\underline{\text { Edition [86] }}\end{array}$ & {$[80][85][86]$} \\
\hline $\begin{array}{l}\text { Open Data } \\
\text { Kit (ODK) }\end{array}$ & $\begin{array}{l}\text { This application was } \\
\text { developed by Get } \\
\text { ODK, an } \\
\text { organization } \\
\text { majoring in data } \\
\text { collection. }\end{array}$ & $\begin{array}{l}\text { ODK software is being employed in the } \\
\text { COVID-19 response for disease surveillance, fast } \\
\text { diagnostics, and vaccine trials. }\end{array}$ & $\begin{array}{l}\text { https://getodk.org/ } \\
\underline{\text { software/ [87] }}\end{array}$ & $\begin{array}{l}\text { https://github.com/getodk/c } \\
\text { ollect [88] }\end{array}$ & {$[80][87][88]$} \\
\hline CommCare & $\begin{array}{l}\text { This software was } \\
\text { developed by } \\
\text { Dimagi, a firm } \\
\text { providing digital data } \\
\text { solutions }\end{array}$ & $\begin{array}{l}\text { Dimagi created a set of pre-built COVID-19 } \\
\text { template applications to help organizations and } \\
\text { governments with their continuing COVID-19 } \\
\text { response operations. }\end{array}$ & $\begin{array}{l}\frac{\text { https://www.dima }}{\text { gi.com/covid-19/ }} \\
\text { [89] }\end{array}$ & $\begin{array}{l}\text { https://github.com/dimagi/ } \\
\text { commcare-hq [90] }\end{array}$ & [80] [89] [90] \\
\hline $\begin{array}{l}\text { KoboToolbo } \\
\mathrm{x}\end{array}$ & $\begin{array}{l}\text { This software was } \\
\text { developed by the } \\
\text { Harvard } \\
\text { Humanitarian } \\
\text { Initiative, an } \\
\text { organization working } \\
\text { on the research and } \\
\text { education of } \\
\text { communities. }\end{array}$ & KoBoToolbox is a data collecting tool. & $\begin{array}{l}\text { https://www.kobot } \\
\text { oolbox.org/ } \\
{[91][92]}\end{array}$ & $\begin{array}{l}\text { https://github.com/kobotoo } \\
\text { lbox [93] }\end{array}$ & $\begin{array}{l}{[80][91][92]} \\
{[93]}\end{array}$ \\
\hline $\begin{array}{l}\text { Fast } \\
\text { automated } \\
\text { detection of } \\
\text { COVID-19 } \\
\text { from } \\
\text { medical } \\
\text { images }\end{array}$ & $\begin{array}{l}\text { This application was } \\
\text { developed by Shuang } \\
\text { Liang \& Huixiang } \\
\text { Liu from School of } \\
\text { Automation and } \\
\text { Electrical } \\
\text { Engineering, } \\
\text { University of Science } \\
\text { and Technology } \\
\text { Beijing; Yu Gu from } \\
\text { School of } \\
\text { Automation, } \\
\text { Guangdong } \\
\text { University of } \\
\text { Petrochemical } \\
\text { Technology, } \\
\text { Maoming; Xiuhua } \\
\text { Guo, Zhiyuan Wu, } \\
\text { Mengyang Liu \& } \\
\text { Lixin Tao } \\
\text { From the Department } \\
\text { of Epidemiology and } \\
\text { Health Statistics, }\end{array}$ & $\begin{array}{l}\text { The software utilizes deep learning framework } \\
\text { (neural network) that identifies COVID-19 from } \\
\text { medical images. }\end{array}$ & & $\begin{array}{l}\text { https://github.com/SHERL } \\
\text { OCKLS/Detection-of- } \\
\text { COVID-19-from-medical- } \\
\underline{\text { images [94] }}\end{array}$ & [95] [94] \\
\hline
\end{tabular}




\begin{tabular}{|c|c|c|c|c|c|}
\hline & $\begin{array}{l}\text { School of Public } \\
\text { Health, Capital } \\
\text { Medical University, } \\
\text { Beijing, China; and } \\
\text { Hongjun Li \& Li } \\
\text { from Beijing Youan } \\
\text { Hospital, Capital } \\
\text { Medical University, } \\
\text { Beijing, China. }\end{array}$ & & & & \\
\hline $\begin{array}{l}\text { The Oxford } \\
\text { Covid-19 } \\
\text { Government } \\
\text { Response } \\
\text { Tracker } \\
\text { (OxCGRT) }\end{array}$ & $\begin{array}{l}\text { This application was } \\
\text { developed by the } \\
\text { Blavatnik School of } \\
\text { Government. }\end{array}$ & $\begin{array}{l}\text { The Oxford Covid-19 Government Response } \\
\text { Tracker (OxCGRT) compiles systematic data on } \\
\text { policy responses taken by countries to combat } \\
\text { COVID-19. Since January 1, 2020, the various } \\
\text { policy reactions have tracked over } 180 \text { nations } \\
\text { and are categorized into } 23 \text { indicators, such as } \\
\text { school closures, travel restrictions, and } \\
\text { vaccination policies. These policies are scored on } \\
\text { a scale to represent the magnitude of government } \\
\text { intervention, and the results are compiled into a } \\
\text { set of policy indices. The data can improve } \\
\text { attempts to combat the epidemic by allowing } \\
\text { decision-makers and citizens to understand } \\
\text { government responses uniformly. }\end{array}$ & $\begin{array}{l}\frac{\text { https://www.bsg.o }}{\text { x.ac.uk/research/r }} \\
\text { esearch- } \\
\text { projects/covid-19- } \\
\text { government- } \\
\text { response-tracker } \\
{[96]}\end{array}$ & $\frac{\text { https://github.com/OxCGR }}{\text { T/covid-policy-tracker [97] }}$ & [98] [96][97] \\
\hline $\begin{array}{l}\text { COVID-19 } \\
\text { Situazione } \\
\text { Italia }\end{array}$ & $\begin{array}{l}\text { This application was } \\
\text { developed by the } \\
\text { Department of Civil } \\
\text { Protection } \\
\text { (Dipartimento della } \\
\text { Protezione Civile) } \\
\text { Angelo Borrelli, } \\
\text { Italy. }\end{array}$ & $\begin{array}{l}\text { This application provides updated Covid-19 data } \\
\text { and visualizations for Italy, including new } \\
\text { confirmed infections, total confirmed infections, } \\
\text { new confirmed deaths, total confirmed deaths, } \\
\text { and recovered cases. The data and visualizations } \\
\text { are provided for the whole country and the } \\
\text { regions. }\end{array}$ & $\frac{\underline{h t t p}: / / \text { arcg.is/C1un }}{\underline{\mathrm{v}}}$ & $\begin{array}{l}\text { https://github.com/pcm- } \\
\underline{\text { dpc/COVID-19 }}[99]\end{array}$ & [3] [99] \\
\hline $\begin{array}{l}\text { Covid } \\
\text { Mobile data }\end{array}$ & $\begin{array}{l}\text { This application was } \\
\text { developed by } \\
\text { COVID19 Mobility } \\
\text { Task Force of the } \\
\text { World Bank }\end{array}$ & $\begin{array}{l}\text { The application uses the data from Mobile } \\
\text { Network Operators (MNOs) to perform analytics. }\end{array}$ & & $\frac{\underline{\text { https://github.com/worldba }}}{\underline{\text { nk/covid-mobile-data }}[100]}$ & [3] [100] \\
\hline $\begin{array}{l}\text { Radar } \\
\text { Covid-19 }\end{array}$ & $\begin{array}{l}\text { The Government of } \\
\text { Spain developed this } \\
\text { application }\end{array}$ & $\begin{array}{l}\text { This application was designed to prevent the } \\
\text { spread of Covid-19. The application anonymizes } \\
\text { users if they have had any contact in the last } 14 \\
\text { days with someone infected with Covid-19 via } \\
\text { low-power Bluetooth technology. }\end{array}$ & $\begin{array}{l}\text { https://radarcovid. } \\
\text { gob.es/ [101] }\end{array}$ & $\frac{\underline{\text { https://github.com/RadarC }}}{\text { OVID/radar-covid-android }}$ & $\begin{array}{l}{[104][101]} \\
{[102][103]}\end{array}$ \\
\hline CovidSafe & $\begin{array}{l}\text { The University of } \\
\text { Washington } \\
\text { developed this } \\
\text { application. }\end{array}$ & $\begin{array}{l}\text { The application was developed to help prevent } \\
\text { the spread of Covid-19 by alerting users about } \\
\text { highly relevant public health announcements, } \\
\text { exposure to COVID-19 and to assist contact } \\
\text { tracing without compromising users' privacy. }\end{array}$ & $\begin{array}{l}\text { https://covidsafe.c } \\
\text { s.washington.edu/ } \\
\text { [105] }\end{array}$ & $\begin{array}{l}\text { https://github.com/CovidSa } \\
\underline{\text { fe }[106]}\end{array}$ & [3] [105] [106] \\
\hline Covid Alert & $\begin{array}{l}\text { Volunteers originally } \\
\text { developed COVID } \\
\text { Alert. The Canadian } \\
\text { Digital Service is } \\
\text { currently developing } \\
\text { its repository. }\end{array}$ & $\begin{array}{l}\text { This application was developed to slow down } \\
\text { Covid-19 infections in Canada. The app notifies } \\
\text { users if someone they were near in the past } 14 \\
\text { days tells the app they tested positive. }\end{array}$ & $\begin{array}{l}\text { https://www.cana } \\
\text { da.ca/en/public- } \\
\text { health/services/dis } \\
\text { eases/coronavirus- } \\
\text { disease-covid- } \\
\text { 19/covid- } \\
\text { alert.html [107] }\end{array}$ & $\begin{array}{l}\text { https://github.com/cds- } \\
\text { snc/covid-alert-app [109] }\end{array}$ & $\begin{array}{l}{[110][107]} \\
{[108][109]}\end{array}$ \\
\hline $\begin{array}{l}\text { erouska- } \\
\text { android }\end{array}$ & $\begin{array}{l}\text { A team of volunteers } \\
\text { initially developed } \\
\text { this application. The } \\
\text { application is } \\
\text { currently developed } \\
\text { and maintained by } \\
\text { the Ministry of } \\
\text { Health in } \\
\text { collaboration with } \\
\text { the National Agency } \\
\text { for Communication }\end{array}$ & $\begin{array}{l}\text { To combat the COVID-19 epidemic, the app } \\
\text { alerts users at risk of spreading the virus. The } \\
\text { software delivers guidance on how to minimize } \\
\text { the spread of the epidemic based on the user's } \\
\text { history of exposure to other potentially } \\
\text { contagious users. }\end{array}$ & $\begin{array}{l}\text { https://erouska.cz/ } \\
\text { [111] }\end{array}$ & $\underline{\underline{\text { https://github.com/covid19 }}}$ & $\begin{array}{l}{[113][111]} \\
{[112]}\end{array}$ \\
\hline
\end{tabular}




\begin{tabular}{|c|c|c|c|c|c|}
\hline & $\begin{array}{l}\text { and Information } \\
\text { Technologies } \\
\text { (NAKIT) of the } \\
\text { Czech Republic as } \\
\text { part of the Smart } \\
\text { Quarantine concept. }\end{array}$ & & & & \\
\hline $\begin{array}{l}\text { COVID-19 } \\
\text { Dashboard }\end{array}$ & $\begin{array}{l}\text { Johns Hopkins } \\
\text { University Centre } \\
\text { developed this } \\
\text { application for } \\
\text { Systems Science and } \\
\text { Engineering. }\end{array}$ & $\begin{array}{l}\text { This is the data repository for the Johns Hopkins } \\
\text { University Centre for Systems Science and } \\
\text { Engineering's } 2019 \text { Novel Coronavirus Visual } \\
\text { Dashboard (JHU CSSE). The ESRI Living Atlas } \\
\text { Team and the Johns Hopkins University Applied } \\
\text { Physics Lab have also contributed to this project } \\
\text { (JHU APL). }\end{array}$ & $\begin{array}{l}\underline{\text { https://www.arcgi }} \\
\text { s.com/apps/opsdas } \\
\underline{\text { hboard/index.html }} \\
\text { \#/bda7594740fd4 } \\
\underline{\text { 0299423467b48e9 }} \\
\underline{\text { ecf6 [114] }}\end{array}$ & $\frac{\text { https://github.com/sidbann }}{\text { et/COVID-19_analysis }}$ & $\begin{array}{l}{[98][114]} \\
{[115][116]}\end{array}$ \\
\hline $\begin{array}{l}\text { Corona- } \\
\text { Warn-App }\end{array}$ & $\begin{array}{l}\text { This application was } \\
\text { developed as an } \\
\text { open-source app by } \\
\text { SAP and Deutsche } \\
\text { Telekom under the } \\
\text { directive by the } \\
\text { government of } \\
\text { Germany. }\end{array}$ & $\begin{array}{l}\text { The Corona-Warn-App was developed with the } \\
\text { goal of preventing the spread of Covid-19. The } \\
\text { app serves as a digital complement to distancing, } \\
\text { hygiene, and wearing masks. Additionally, it } \\
\text { provides a functionality to add a user's digital } \\
\text { vaccination certificate to prove their vaccination } \\
\text { status. }\end{array}$ & $\frac{\underline{\text { https://www.coron }}}{\text { awarn.app/en/ }}$ & $\begin{array}{l}\frac{\text { https://github.com/corona- }}{\text { warn-app/cwa-app-android }} \\
{[118]}\end{array}$ & $\begin{array}{l}{[119][117]} \\
{[118]}\end{array}$ \\
\hline $\begin{array}{l}\text { TraceTogeth } \\
\text { er }\end{array}$ & $\begin{array}{l}\text { The Singapore } \\
\text { Government } \\
\text { Technology Agency } \\
\text { developed this } \\
\text { application. }\end{array}$ & $\begin{array}{l}\text { Through community-driven contact tracing, } \\
\text { TraceTogether supports Singapore's efforts to } \\
\text { combat the spread of COVID-19. One can use } \\
\text { the app to see or display their COVID Health } \\
\text { Status based on their immunization and test } \\
\text { results. }\end{array}$ & $\frac{\text { https://www.tracet }}{\text { ogether.gov.sg/ }}$ & $\frac{\text { https://github.com/OpenTr }}{\underline{\text { ace-Community [121] }}}$ & $\begin{array}{l}{[122][120]} \\
{[121]}\end{array}$ \\
\hline $\begin{array}{l}\text { NZ COVID } \\
\text { Tracer }\end{array}$ & $\begin{array}{l}\text { The New Zealand } \\
\text { Ministry of Health } \\
\text { developed this } \\
\text { application }\end{array}$ & $\begin{array}{l}\text { The app helps contact tracing go faster by } \\
\text { creating a private digital diary of the places you } \\
\text { visit. Users Scan the official QR codes wherever } \\
\text { they see them and add manual entries for their } \\
\text { visits to other places. }\end{array}$ & $\begin{array}{l}\text { https://www.healt } \\
\text { h.govt.nz/our- } \\
\text { work/diseases- } \\
\text { and- } \\
\text { conditions/covid- } \\
\underline{\text { 19-novel- }} \\
\text { coronavirus/covid } \\
\underline{\text {-19-resources- }} \\
\text { and-tools/nz- } \\
\text { covid-tracer-app } \\
{[123]}\end{array}$ & $\frac{\text { https://github.com/minheal }}{\text { thnz/nzcovidtracer-app }}$ & $\begin{array}{l}{[126][123]} \\
{[124][125]}\end{array}$ \\
\hline $\begin{array}{l}\text { VigilantGan } \\
\text { try }\end{array}$ & $\begin{array}{l}\text { This an automated } \\
\text { contactless gantry } \\
\text { system developed by } \\
\text { GovTech's Data } \\
\text { Science and Artificial } \\
\text { Intelligence Division } \\
\text { (DSAID) }\end{array}$ & $\begin{array}{l}\text { VigilantGantry is an open-source implementation } \\
\text { of an AI-driven automated temperature screening } \\
\text { gantry that improves the rate of contactless } \\
\text { screening by augmenting existing thermal } \\
\text { systems. VigilantGantry is excellent for } \\
\text { automatically scanning high-traffic sites for } \\
\text { symptomatic COVID-19 patients. It helps ground } \\
\text { crews keep on the lookout for COVID-19. }\end{array}$ & & $\begin{array}{l}\text { https://github.com/dsaidgo } \\
\text { vsg/vigilantgantry [127] }\end{array}$ & [128] [127] \\
\hline $\begin{array}{l}\text { lancet- } \\
\text { covid-19- } \\
\text { database }\end{array}$ & Developed by Lancet & $\begin{array}{l}\text { The Lancet COVID-19 Database gives } \\
\text { users access to the most up-to-date information } \\
\text { on COVID-19, such as cases, deaths, recoveries, } \\
\text { testing, and other useful indicators for tracking } \\
\text { the pandemic's spread and response. }\end{array}$ & & $\frac{\text { https://github.com/sdsna/la }}{\text { ncet-covid-19-database }}$ & [131] [130] \\
\hline $\begin{array}{l}\text { Covid19Can } \\
\text { ada }\end{array}$ & $\begin{array}{l}\text { SDSN developed this } \\
\text { application }\end{array}$ & $\begin{array}{l}\text { This shiny app provides a forecast of Covid-19 } \\
\text { cases and Covid-19 information in Canada, } \\
\text { including the confirmed new cases of infections, } \\
\text { confirmed deaths, and recovery rate. Apart from } \\
\text { forecasting, the application utilizes plots to } \\
\text { visualize the disease's ten-day forecasts and the } \\
\text { accumulated and confirmed data. }\end{array}$ & $\begin{array}{l}\frac{\text { https://art- }}{\text { bd.shinyapps.io/co }} \\
\frac{\text { vid19canada/ }}{[132]}\end{array}$ & $\begin{array}{l}\text { https://github.com/ccodwg/ } \\
\text { Covid19Canada [133] }\end{array}$ & [3] [132] [133] \\
\hline COVI-ML & $\begin{array}{l}\text { This respiratory was } \\
\text { developed by the } \\
\text { Quebec Artificial } \\
\text { Intelligence Institute }\end{array}$ & $\begin{array}{l}\text { COVI-ML is the Risk model training code for the } \\
\text { Covid-19 tracing application. Its repository } \\
\text { provides models, infrastructure, and datasets for } \\
\text { training deep-learning-based predictors of } \\
\text { COVID-19 infectiousness as used in Proactive } \\
\text { Contact Tracing. }\end{array}$ & & $\frac{\text { https://github.com/mila- }}{\text { iqia/COVI-ML [134] }}$ & {$[3][134]$} \\
\hline
\end{tabular}




\begin{tabular}{|c|c|c|c|c|c|}
\hline $\begin{array}{l}\text { Covid-19 } \\
\text { model }\end{array}$ & $\begin{array}{l}\text { Imperial College } \\
\text { London developed } \\
\text { this application/code. }\end{array}$ & $\begin{array}{l}\text { This code was applied in modeling estimated } \\
\text { deaths and infections for COVID-19 from the } \\
\text { study "Estimating the effects of non- } \\
\text { pharmaceutical interventions on COVID-19 in } \\
\text { Europe "done by Flaxman et al. (2020) [136] }\end{array}$ & & $\begin{array}{l}\underline{\text { https://github.com/Imperial }} \\
\underline{\underline{\text { dellegeLondon/covid19mo }}} \\
\underline{\underline{\text { d135] }}}\end{array}$ & [136] [135] \\
\hline $\begin{array}{l}\text { The COVID } \\
\text { Tracking } \\
\text { Project }\end{array}$ & $\begin{array}{l}\text { Alexis Madrigal } \\
\text { created this project } \\
\text { through a } \\
\text { collaborative } \\
\text { volunteer-run effort } \\
\text { to track the ongoing } \\
\text { COVID-19 pandemic }\end{array}$ & $\begin{array}{l}\text { This project collects and publishes data required } \\
\text { to understand the COVID-19 outbreak in the } \\
\text { United States. All } 50 \text { states, five territories, and } \\
\text { the District of Columbia participate in the Covid } \\
\text { tracking project, which will collect data on } \\
\text { COVID-19 testing and patient outcomes. }\end{array}$ & $\frac{\text { https://covidtracki }}{\underline{\text { ng.com/ [137] }}}$ & $\begin{array}{l}\text { https://github.com/COVID } \\
\underline{\text { 19Tracking [138] }}\end{array}$ & $\begin{array}{l}{[139][137]} \\
{[138]}\end{array}$ \\
\hline Covidmx & $\begin{array}{l}\text { Covidmx was } \\
\text { developed by } \\
\text { Federico Garza }\end{array}$ & $\begin{array}{l}\text { The API was developed to handle Covid-19 open } \\
\text { data provided by the Mexican Dirección General } \\
\text { de Epidemiología. }\end{array}$ & & $\begin{array}{l}\text { https://github.com/Federic } \\
\text { oGarza/covidmx }[140]\end{array}$ & [141] [140] \\
\hline $\begin{array}{l}\text { Covid19- } \\
\text { Scenarios }\end{array}$ & $\begin{array}{l}\text { Neherlab developed } \\
\text { this tool }\end{array}$ & $\begin{array}{l}\text { The Covid-19 Scenarios provide Models of } \\
\text { generating trajectories for COVID-19 outbreak } \\
\text { and hospital demand. The functioning of this tool } \\
\text { is based on the SIR model, which simulates a } \\
\text { COVID19 outbreak. }\end{array}$ & $\frac{\underline{\text { https://covid19- }}}{\frac{\text { scenarios.org/ }}{[142]}}$ & $\begin{array}{l}\text { https://github.com/neherlab } \\
\text { /covid19_scenarios [143] }\end{array}$ & [3] [142] [143] \\
\hline $\begin{array}{l}\text { covid-chest- } \\
\text { imaging- } \\
\text { database }\end{array}$ & $\begin{array}{l}\text { This database was } \\
\text { developed by NHSX } \\
\text { and the British } \\
\text { Society of Thoracic } \\
\text { Imaging (BSTI). } \\
\text { NHSX is a joint unit } \\
\text { of National Health } \\
\text { Service (NHS) } \\
\text { England and the } \\
\text { Department of Health } \\
\text { and Social Care, } \\
\text { supporting local NHS } \\
\text { and care } \\
\text { organizations. }\end{array}$ & $\begin{array}{l}\text { The database was developed with a respiratory } \\
\text { containing tooling related to the NHSX National } \\
\text { COVID-19 Chest Image Database (NCCID) to } \\
\text { promote research projects in response to the } \\
\text { COVID-19 pandemic. }\end{array}$ & & $\frac{\frac{\text { https://github.com/nhsx/co }}{\text { vid-chest-imaging-database }}}{\text { [144] }}$ & [145] [144] \\
\hline $\begin{array}{l}\text { Covid-pass- } \\
\text { verifier }\end{array}$ & $\begin{array}{l}\text { This is an application } \\
\text { developed by NHSX }\end{array}$ & $\begin{array}{l}\text { The COVID Pass Verifier app is the official NHS } \\
\text { COVID Pass Verifier for England and Wales. } \\
\text { The app is a safe and secure way to check if } \\
\text { someone has been appropriately vaccinated } \\
\text { against COVID-19, has had a negative test, or } \\
\text { has recovered from COVID-19. }\end{array}$ & $\begin{array}{l}\text { https://www.nhsx. } \\
\text { nhs.uk/covid-19- } \\
\text { response/nhs- } \\
\text { covid-pass- } \\
\text { verifier- } \\
\text { app/international- } \\
\text { covid-pass- } \\
\text { verifier-app-user- } \\
\text { guide/ [146] }\end{array}$ & $\begin{array}{l}\underline{\text { https://github.com/nhsx/co }} \\
\text { vid-pass-verifier [148] }\end{array}$ & $\begin{array}{l}{[145][146]} \\
{[147][148]}\end{array}$ \\
\hline Covasim & $\begin{array}{l}\text { The Institute for } \\
\text { Disease Modelling } \\
\text { developed this } \\
\text { simulator }\end{array}$ & $\begin{array}{l}\text { Covasim is a stochastic agent-based simulator for } \\
\text { performing COVID-19 analyses. }\end{array}$ & & $\begin{array}{l}\underline{\text { https://github.com/Institute }} \\
\underline{\text { forDiseaseModeling/covasi }} \\
\underline{m} \text { [149] }\end{array}$ & [150] [149] \\
\hline $\begin{array}{l}\text { covid-19 } \\
\text { Dashboard }\end{array}$ & $\begin{array}{l}\text { Greg Rafferty } \\
\text { developed Covid-19 } \\
\text { dashboard }\end{array}$ & $\begin{array}{l}\text { This is a web dashboard developed to monitor } \\
\text { the COVID-19 pandemic. The data used is } \\
\text { obtained from Johns Hopkins Center for Systems } \\
\text { Science and Engineering. }\end{array}$ & $\begin{array}{l}\underline{\text { https://covid-19- }} \\
\underline{\text { raffg.herokuapp.c }} \\
\underline{\text { om/ }[151]}\end{array}$ & $\underline{\underline{\text { https://github.com/raffg/co }}}$ & [3] [152] [151] \\
\hline $\begin{array}{l}\text { Covid-19 } \\
\text { R/Python } \\
\text { scripts }\end{array}$ & $\begin{array}{l}\text { Developed by } \\
\text { QuKunLa; a } \\
\text { Laboratory of } \\
\text { Immunogenomics } \\
\text { and Precision } \\
\text { Medicine, University } \\
\text { of Science and } \\
\text { Technology of China }\end{array}$ & $\begin{array}{l}\text { These are R/Python scripts to analyze single-cell } \\
\text { RNA-sequence data from COVID-19 patients. }\end{array}$ & & $\underline{\underline{\text { https://github.com/QuKun }}}$ & [3] [153] \\
\hline $\begin{array}{l}\text { COVID-19- } \\
\text { CT-CXR }\end{array}$ & $\begin{array}{l}\text { COVID-19-CT-CXR } \\
\text { was developed by } \\
\text { Peng et al. and } \\
\text { Intramural Research } \\
\text { Programs of the }\end{array}$ & $\begin{array}{l}\text { This is a public database of COVID-19 CXR and } \\
\text { CT images, which are automatically extracted } \\
\text { from COVID-19-relevant articles from the } \\
\text { PubMed Central Open Access (PMC-OA) }\end{array}$ & & $\frac{\frac{\text { https://github.com/ncbi- }}{\text { nlp/COVID-19-CT-CXR }}}{[154]}$ & [155] [154] \\
\hline
\end{tabular}




\begin{tabular}{|c|c|c|c|c|c|}
\hline & $\begin{array}{l}\text { National Institutes of } \\
\text { Health, National } \\
\text { Library of Medicine } \\
\text { and Clinical Centre. }\end{array}$ & Subset. & & & \\
\hline $\begin{array}{l}\text { covid19- } \\
\text { healthsyste } \\
\text { mcapacity }\end{array}$ & $\begin{array}{l}\text { This project was } \\
\text { developed by the } \\
\text { CovidCareMap } \\
\text { organization }\end{array}$ & $\begin{array}{l}\text { This application assists in better understanding, } \\
\text { anticipating, and acting to support and ramp up } \\
\text { our health systems' capacity (beds, staffing, } \\
\text { ventilators, supplies) to effectively care for a } \\
\text { rapidly growing number of active COVID19 } \\
\text { patients in need of hospitalization and intensive } \\
\text { (ICU) care. }\end{array}$ & & $\begin{array}{l}\text { https://github.com/covidcar } \\
\text { emap/covid19- } \\
\text { healthsystemcapacity [156] }\end{array}$ & [3] [156] \\
\hline CV19 Index & $\begin{array}{l}\text { The Global Loop } \\
\text { team developed this } \\
\text { model }\end{array}$ & $\begin{array}{l}\text { The COVID-19 Vulnerability Index (CV19 } \\
\text { Index) is a predictive model that identifies } \\
\text { persons who are more susceptible to COVID-19 } \\
\text { severe problems. The CV19 Index is designed to } \\
\text { assist hospitals, federal, state, and local public } \\
\text { health agencies, and other healthcare } \\
\text { organizations in identifying, planning for, } \\
\text { responding to, and reducing COVID-19's impact } \\
\text { in their areas. }\end{array}$ & $\frac{\frac{\text { https://www.close }}{\text { dloop.ai/covid-19- }}}{\underline{\text { index [157] }}}$ & $\begin{array}{l}\text { https://github.com/closedlo } \\
\text { op-ai/cv19index [158] }\end{array}$ & $\begin{array}{l}{[159][157]} \\
{[158]}\end{array}$ \\
\hline $\begin{array}{l}\text { OpenABM- } \\
\text { Covid19 }\end{array}$ & $\begin{array}{l}\text { This model was } \\
\text { developed by the } \\
\text { Pathogen Dynamics } \\
\text { Group of Oxford Big } \\
\text { Data Institute. }\end{array}$ & $\begin{array}{l}\text { OpenABM-Covid } 19 \text { is an agent-based model } \\
\text { (ABM) that was created to model the spread of } \\
\text { Covid-19 in a city and investigate the impact of } \\
\text { passive and active intervention measures. }\end{array}$ & & $\begin{array}{l}\text { https://github.com/BDI- } \\
\text { pathogens/OpenABM- } \\
\text { Covid19 [160] }\end{array}$ & [3] [160] \\
\hline $\begin{array}{l}\text { COVID-19 } \\
\text { vaccination } \\
\text { slot booking } \\
\text { script }\end{array}$ & $\begin{array}{l}\text { PythonRepo } \\
\text { developed this script. }\end{array}$ & Is used to automate covid vaccination booking. & $\begin{array}{l}\text { https://pythonrepo } \\
\text {.com/repo/pallupz } \\
\text {-covid-vaccine- } \\
\text { booking [161] }\end{array}$ & $\begin{array}{l}\text { https://pythonrepo.com/rep } \\
\text { o/pallupz-covid-vaccine- } \\
\underline{\text { booking [162] }}\end{array}$ & $\begin{array}{l}{[159][161]} \\
{[162]}\end{array}$ \\
\hline
\end{tabular}

TABLE II. COVID-19 DATASETS SUMMARY TABLE

\begin{tabular}{|c|c|c|c|c|}
\hline $\begin{array}{l}\text { Developer/Industry/Academia/ } \\
\text { University/Organization Details }\end{array}$ & Dataset Summary & Dataset Usage & Weblink to the Dataset & $\begin{array}{l}\text { Referen } \\
\text { ces }\end{array}$ \\
\hline \multirow[t]{7}{*}{ Our World in Data } & $\begin{array}{l}\text { Data on COVID-19 } \\
\text { vaccinations that include } \\
\text { country-by country } \\
\text { statistics of the COVID-19 } \\
\text { vaccines administered to } \\
\text { date. }\end{array}$ & $\begin{array}{l}\text { Vaccine outreach } \\
\text { program. }\end{array}$ & https://ourworldindata.org/covid-vaccinations. [164] & $\begin{array}{l}{[163][16} \\
4]\end{array}$ \\
\hline & $\begin{array}{l}\text { Data on COVID-19 } \\
\text { confirmed deaths per } \\
\text { country. }\end{array}$ & $\begin{array}{l}\text { Effects of testing, } \\
\text { managing, } \\
\text { hospitalization. }\end{array}$ & https://ourworldindata.org/covid-deaths.[165] & $\begin{array}{l}{[362][16} \\
5][394]\end{array}$ \\
\hline & $\begin{array}{l}\text { Global data of confirmed } \\
\text { COVID-19 cases }\end{array}$ & $\begin{array}{l}\text { Effect on travel } \\
\text { restriction, } \\
\text { intervention } \\
\text { programs. }\end{array}$ & https://ourworldindata.org/covid-cases. [166] & $\begin{array}{l}{[393][16} \\
6]\end{array}$ \\
\hline & $\begin{array}{l}\text { Data on COVID-19 } \\
\text { testing, i.e., positivity rate, } \\
\text { contact tracing, tests } \\
\text { performed per day }\end{array}$ & $\begin{array}{l}\text { Pandemic } \\
\text { preventive measures }\end{array}$ & https://ourworldindata.org/coronavirus-testing. [167] & $\begin{array}{l}{[393][16} \\
7]\end{array}$ \\
\hline & $\begin{array}{l}\text { Data on COVID-19 } \\
\text { hospitalization }\end{array}$ & $\begin{array}{l}\text { Monitoring cases to } \\
\text { improve impact on } \\
\text { available resources. }\end{array}$ & https://ourworldindata.org/covid-hospitalizations.[168] & $\begin{array}{l}{[392]} \\
{[168]}\end{array}$ \\
\hline & COVID-19 mortality risks & $\begin{array}{l}\text { Segregation of age } \\
\text { groups that may be } \\
\text { at risk of dying from } \\
\text { the disease. }\end{array}$ & https://ourworldindata.org/mortality-risk-covid. [169] & $\begin{array}{l}\text { [393][16 } \\
9][170]\end{array}$ \\
\hline & $\begin{array}{l}\text { Excess mortality due to } \\
\text { COVID-19 }\end{array}$ & $\begin{array}{l}\text { Segregation of age } \\
\text { groups that may be } \\
\text { at risk of dying from } \\
\text { the disease, and }\end{array}$ & https://ourworldindata.org/excess-mortality-covid. [171] & $\begin{array}{l}{[393][17} \\
1]\end{array}$ \\
\hline
\end{tabular}




\begin{tabular}{|c|c|c|c|c|}
\hline & & $\begin{array}{l}\text { other accelerating } \\
\text { factors. }\end{array}$ & & \\
\hline & $\begin{array}{l}\text { Policy responses to the } \\
\text { COVID-19 pandemic }\end{array}$ & $\begin{array}{l}\text { Government } \\
\text { interventions to curb } \\
\text { the spread of the } \\
\text { virus. }\end{array}$ & https://ourworldindata.org/policy-responses-covid. [172] & $\begin{array}{l}{[393][17} \\
2][173]\end{array}$ \\
\hline $\begin{array}{l}\text { The Johns Hopkins University } \\
\text { Center for Systems Science and } \\
\text { Engineering [JHU CCSE] }\end{array}$ & $\begin{array}{l}\text { COVID-19 } \\
\text { Epidemiological Data }\end{array}$ & $\begin{array}{l}\text { For segmentation of } \\
\text { COVID-19 cases } \\
\text { based on } \\
\text { epidemiological } \\
\text { characteristics. }\end{array}$ & $\begin{array}{l}\text { https://data.humdata.org/dataset/novel-coronavirus-2019- } \\
\text { ncov-cases. [174] }\end{array}$ & {$[174]$} \\
\hline OCHA & $\begin{array}{l}\text { COVID-19 number of } \\
\text { confirmed cases, deaths, } \\
\text { and recoveries by the } \\
\text { province in Indonesia }\end{array}$ & $\begin{array}{l}\text { Mobility } \\
\text { transmission } \\
\text { analysis. }\end{array}$ & $\begin{array}{l}\text { https://data.humdata.org/dataset/indonesia-covid-19- } \\
\text { cases-recoveries-and-deaths-per-province.[175] }\end{array}$ & {$[175]$} \\
\hline World Health Organization & $\begin{array}{l}\text { COVID-19 cases and } \\
\text { deaths }\end{array}$ & $\begin{array}{l}\text { Mobility } \\
\text { transmission and } \\
\text { mortality analysis. }\end{array}$ & $\begin{array}{l}\text { https://data.humdata.org/dataset/coronavirus-covid-19- } \\
\text { cases-and-deaths.[176] }\end{array}$ & {$[176]$} \\
\hline $\begin{array}{l}\text { Blavatnik School of Government, } \\
\text { University of Oxford }\end{array}$ & $\begin{array}{l}\text { OXFORD COVID-19 } \\
\text { Government Response } \\
\text { Stringency index }\end{array}$ & $\begin{array}{l}\text { Government } \\
\text { measures }\end{array}$ & $\begin{array}{l}\text { https://data.humdata.org/dataset/oxford-covid-19- } \\
\text { government-response-tracker. [177] }\end{array}$ & {$[177]$} \\
\hline HDX & COVID-19 Vaccinations & $\begin{array}{l}\text { Rate of vaccine } \\
\text { drives }\end{array}$ & $\begin{array}{l}\text { https://data.humdata.org/dataset/covid-19-vaccinations. } \\
\text { [178] }\end{array}$ & [178] \\
\hline World Food Program & $\begin{array}{l}\text { COVID-19 global airline } \\
\text { information and travel } \\
\text { restriction }\end{array}$ & Global Monitoring. & $\begin{array}{l}\text { https://data.humdata.org/dataset/covid-19-global-travel- } \\
\text { restrictions-and-airline-information. [179] }\end{array}$ & {$[179]$} \\
\hline HDX & $\begin{array}{l}\text { COVID-19 cases and } \\
\text { deaths in the United States }\end{array}$ & $\begin{array}{l}\text { Reporting cases at a } \\
\text { national level. }\end{array}$ & https://data.humdata.org/dataset/nyt-covid-19-data. [180] & {$[180]$} \\
\hline HDX & $\begin{array}{l}\text { Total number of COVID- } \\
19 \text { tests performed per } \\
\text { country }\end{array}$ & Monitoring cases & $\begin{array}{l}\text { https://data.humdata.org/dataset/total-covid-19-tests- } \\
\text { performed-by-country. [181] }\end{array}$ & [181] \\
\hline UNESCO & Global school closures & Area segmentation & $\begin{array}{l}\text { https://data.humdata.org/dataset/global-school-closures- } \\
\text { covid19. [182] }\end{array}$ & {$[182]$} \\
\hline Meta & $\begin{array}{l}\text { FAIR COVID-19 US } \\
\text { County Forecast }\end{array}$ & $\begin{array}{l}\text { Country-level } \\
\text { forecast. }\end{array}$ & https://data.humdata.org/dataset/fair-covid-dataset.[183] & [183] \\
\hline CARE Bangladesh & $\begin{array}{l}\text { District Wise Quarantine } \\
\text { for COVID-19 }\end{array}$ & $\begin{array}{l}\text { Reporting cases at a } \\
\text { national level. }\end{array}$ & $\begin{array}{l}\text { https://data.humdata.org/dataset/district-wise-quarantine- } \\
\text { for-covid-19. [184] }\end{array}$ & {$[184]$} \\
\hline HDX & $\begin{array}{l}\text { COVID-19 Impact on } \\
\text { Humanitarian Operations } \\
\text { Data Viz inputs }\end{array}$ & $\begin{array}{l}\text { Reporting } \\
\text { humanitarian } \\
\text { activities at a } \\
\text { national level. }\end{array}$ & $\begin{array}{l}\text { https://data.humdata.org/dataset/covid-19-data-visual- } \\
\text { inputs. [185] }\end{array}$ & [185] \\
\hline OCHA Venezuela & $\begin{array}{l}\text { COVID-19 sub-national } \\
\text { data }\end{array}$ & $\begin{array}{l}\text { Reporting cases at a } \\
\text { national level. }\end{array}$ & $\begin{array}{l}\text { https://data.humdata.org/dataset/corona-virus-covid-19- } \\
\text { cases-and-deaths-in-venezuela.[186] }\end{array}$ & {$[186]$} \\
\hline OCHA FISS & $\begin{array}{l}\text { Global Humanitarian } \\
\text { Operational Presence } \\
\text { Who, What, Where [3W] } \\
\text { Portal }\end{array}$ & $\begin{array}{l}\text { Reporting } \\
\text { humanitarian } \\
\text { activities at a global } \\
\text { level. }\end{array}$ & $\begin{array}{l}\text { https://data.humdata.org/dataset/ocha-global- } \\
\text { humanitarian-operational-presence-who-what-where-3w- } \\
\text { portal.[187] }\end{array}$ & {$[187]$} \\
\hline ACAPS & $\begin{array}{l}\text { COVID-19 Government } \\
\text { Measures Dataset }\end{array}$ & $\begin{array}{l}\text { Reporting } \\
\text { government } \\
\text { measures at a global } \\
\text { level. }\end{array}$ & $\begin{array}{l}\text { https://data.humdata.org/dataset/acaps-covid19- } \\
\text { government-measures-dataset.[188] }\end{array}$ & [188] \\
\hline HDX & $\begin{array}{l}\text { Europe COVID-19 } \\
\text { subnational cases }\end{array}$ & $\begin{array}{l}\text { COVID-19 infected } \\
\text { area segmentation. }\end{array}$ & $\begin{array}{l}\text { https://data.humdata.org/dataset/europe-covid-19- } \\
\text { subnational-cases. [190] }\end{array}$ & {$[190]$} \\
\hline OCHA Philippines & $\begin{array}{l}\text { Philippines COVID-19 } \\
\text { response. }\end{array}$ & $\begin{array}{l}\text { Reporting } \\
\text { government } \\
\text { measures at a } \\
\text { national level. }\end{array}$ & $\begin{array}{l}\text { https://data.humdata.org/dataset/philippines-covid-19- } \\
\text { response-who-does-what-where. [191] }\end{array}$ & [191] \\
\hline
\end{tabular}




\begin{tabular}{|c|c|c|c|c|}
\hline Code for Venezuela & $\begin{array}{l}\text { COVID-19 education } \\
\text { impact survey }\end{array}$ & $\begin{array}{l}\text { Monitoring impact } \\
\text { on a national level }\end{array}$ & $\begin{array}{l}\text { https://data.humdata.org/dataset/open_one_time_covid_e } \\
\text { ducation_impact. } \\
\text { [192] }\end{array}$ & {$[192]$} \\
\hline iMMAP & Google mobility report & $\begin{array}{l}\text { Mobility } \\
\text { transmission } \\
\text { analysis. }\end{array}$ & $\begin{array}{l}\text { https://data.humdata.org/dataset/google-mobility-report. } \\
\text { [193] }\end{array}$ & {$[193]$} \\
\hline $\begin{array}{l}\text { Humanitarian Emergency Report } \\
\text { Africa [HERA] }\end{array}$ & $\begin{array}{l}\text { Subnational data on Covid } \\
19 \text { cases per day }\end{array}$ & $\begin{array}{l}\text { COVID-19 infected } \\
\text { area segmentation. }\end{array}$ & $\begin{array}{l}\text { https://data.humdata.org/dataset/nigeria_covid19_subnati } \\
\text { onal.[194] }\end{array}$ & {$[194]$} \\
\hline HDX & $\begin{array}{l}\text { Worldwide geographic } \\
\text { distribution of COVID-19 } \\
\text { cases }\end{array}$ & $\begin{array}{l}\text { COVID-19 infected } \\
\text { area segmentation. }\end{array}$ & https://data.humdata.org/dataset/ecdc-covid-19. [195] & {$[195]$} \\
\hline World Health Organization & $\begin{array}{l}\text { Immunization campaigns } \\
\text { impacted due to COVID- } \\
19 .\end{array}$ & $\begin{array}{l}\text { Mobility } \\
\text { transmission } \\
\text { analysis }\end{array}$ & $\begin{array}{l}\text { https://data.humdata.org/dataset/immunization- } \\
\text { campaigns-impacted. [196] }\end{array}$ & {$[196]$} \\
\hline HDX & $\begin{array}{l}\text { Excess mortality during } \\
\text { COVID-19 pandemic }\end{array}$ & $\begin{array}{l}\text { Segregation of age } \\
\text { groups that may be } \\
\text { at risk of dying from } \\
\text { the disease. }\end{array}$ & $\begin{array}{l}\text { https://data.humdata.org/dataset/financial-times-excess- } \\
\text { mortality-during-covid-19-pandemic-data. [197] }\end{array}$ & {$[197]$} \\
\hline HDX & $\begin{array}{l}\text { COVID-19 subnational } \\
\text { cases in Palestine }\end{array}$ & $\begin{array}{l}\text { Reporting cases at a } \\
\text { national level. }\end{array}$ & $\begin{array}{l}\text { https://data.humdata.org/dataset/state-of-palestine- } \\
\text { coronavirus-covid-19-subnational-cases.[198] }\end{array}$ & [198] \\
\hline Meta & $\begin{array}{l}\text { Impact survey and trends } \\
\text { on COVID-19 }\end{array}$ & $\begin{array}{l}\text { Reporting cases at a } \\
\text { national level. }\end{array}$ & $\begin{array}{l}\text { https://data.humdata.org/dataset/covid-19-symptom-map. } \\
\text { [199] }\end{array}$ & {$[199]$} \\
\hline HDX & $\begin{array}{l}\text { COVID-19 vaccine doses } \\
\text { are given to humanitarian } \\
\text { resource plan countries }\end{array}$ & $\begin{array}{l}\text { Forecasts on dose } \\
\text { availability and } \\
\text { actual deliveries }\end{array}$ & $\begin{array}{l}\text { https://data.humdata.org/dataset/covid-19-vaccine-doses- } \\
\text { in-hrp-countries. [200] }\end{array}$ & {$[200]$} \\
\hline World Bank Group & $\begin{array}{l}\text { World Bank indicators of } \\
\text { interest to the COVID-19 } \\
\text { outbreak }\end{array}$ & $\begin{array}{l}\text { Data for use in } \\
\text { response, modeling } \\
\text { analysis }\end{array}$ & $\begin{array}{l}\text { https://data.humdata.org/dataset/world-bank-indicators- } \\
\text { of-interest-to-the-covid-19-outbreak.[201] }\end{array}$ & {$[201]$} \\
\hline Global Health 50/50 & $\begin{array}{l}\text { Gender and COVID-19 } \\
\text { project }\end{array}$ & $\begin{array}{l}\text { Exploring how } \\
\text { gender may be } \\
\text { driving the higher } \\
\text { proportion of } \\
\text { reported deaths in } \\
\text { men among } \\
\text { confirmed cases so } \\
\text { far. }\end{array}$ & http://globalhealth5050.org/covid19[202] & {$[202]$} \\
\hline World Bank Group & $\begin{array}{l}\text { Harmonized data on } \\
\text { Household COVID-19 } \\
\text { monitoring surveys }\end{array}$ & $\begin{array}{l}\text { Data analysis and } \\
\text { trend checking }\end{array}$ & $\begin{array}{l}\text { https://data.humdata.org/dataset/harmonized-covid-19- } \\
\text { household-monitoring-surveys[203] }\end{array}$ & {$[203]$} \\
\hline $\begin{array}{l}\text { Humanitarian Emergency } \\
\text { Response Africa [HERA] }\end{array}$ & $\begin{array}{l}\text { African continent Covid } \\
19 \text { cases }\end{array}$ & $\begin{array}{l}\text { Data analysis and } \\
\text { trendsetting }\end{array}$ & $\begin{array}{l}\text { https://data.humdata.org/dataset/covid19_africa_continen } \\
\text { tal_infections-recoveries-deaths[204] }\end{array}$ & {$[204]$} \\
\hline Dalberg & $\begin{array}{l}\text { Developing countries' } \\
\text { government action on } \\
\text { COVID-19 }\end{array}$ & $\begin{array}{l}\text { non-pharmaceutical } \\
\text { interventions }\end{array}$ & $\begin{array}{l}\text { https://data.humdata.org/dataset/government-actions-on- } \\
\text { covid-19[205] }\end{array}$ & {$[205]$} \\
\hline Meta & $\begin{array}{l}\text { Survey on preventative } \\
\text { health }\end{array}$ & $\begin{array}{l}\text { Monitor and } \\
\text { understand people's } \\
\text { knowledge and } \\
\text { practices about } \\
\text { COVID-19 to } \\
\text { improve } \\
\text { communications and } \\
\text { their response to the } \\
\text { pandemic. }\end{array}$ & $\begin{array}{l}\text { https://data.humdata.org/dataset/preventive-health- } \\
\text { survey[206] }\end{array}$ & [206] \\
\hline $\begin{array}{l}\text { International } \\
\text { Organization for Migration }\end{array}$ & $\begin{array}{l}\text { Information on } \\
\text { populations within the Far } \\
\text { North region of Cameroon }\end{array}$ & $\begin{array}{l}\text { Providing regular, } \\
\text { accurate, and } \\
\text { updated data to } \\
\text { better support the } \\
\text { response of the } \\
\text { Government of } \\
\text { Cameroon and the } \\
\text { humanitarian }\end{array}$ & $\begin{array}{l}\text { https://data.humdata.org/dataset/cameroon-baseline- } \\
\text { assessment-data-iom-dtm[207] }\end{array}$ & [207] \\
\hline
\end{tabular}




\begin{tabular}{|c|c|c|c|c|}
\hline & & community. & & \\
\hline HDX & Covax round 6 allocations & $\begin{array}{l}\text { Monitoring of } \\
\text { Covax vaccine } \\
\text { allocations }\end{array}$ & $\begin{array}{l}\text { https://data.humdata.org/dataset/covax-round-6- } \\
\text { allocations.[208] }\end{array}$ & [208] \\
\hline $\begin{array}{l}\text { Humanitarian Emergency } \\
\text { Response Africa }\end{array}$ & $\begin{array}{l}\text { COVID-19 subnational } \\
\text { data in Burkina Faso }\end{array}$ & $\begin{array}{l}\text { Reporting Covid } \\
\text { data at National } \\
\text { level }\end{array}$ & $\begin{array}{l}\text { https://data.humdata.org/dataset/burkinafaso_covid19_su } \\
\text { bnational[209] }\end{array}$ & [209] \\
\hline Metabiota & $\begin{array}{l}\text { Spatiotemporal data for } \\
\text { COVID-19 deaths and } \\
\text { cases. }\end{array}$ & $\begin{array}{l}\text { Data analysis and } \\
\text { monitoring }\end{array}$ & $\begin{array}{l}\text { https://data.humdata.org/dataset/2019-novel-coronavirus- } \\
\text { cases[210] }\end{array}$ & [210] \\
\hline HDX & $\begin{array}{l}\text { COVID-19 subnational } \\
\text { data for Afghanistan }\end{array}$ & $\begin{array}{l}\text { Data analysis and } \\
\text { reporting on a } \\
\text { national level }\end{array}$ & $\begin{array}{l}\text { https://data.humdata.org/dataset/afghanistan-covid-19- } \\
\text { statistics-per-province[211] }\end{array}$ & [211] \\
\hline Cuebiq Inc & $\begin{array}{l}\text { COVID-19 mobility data } \\
\text { for Italy }\end{array}$ & $\begin{array}{l}\text { Monitoring mobility } \\
\text { changes in Italy } \\
\text { since lockdown }\end{array}$ & $\begin{array}{l}\text { https://data.humdata.org/dataset/covid-19-mobility- } \\
\text { italy[212] }\end{array}$ & [212] \\
\hline $\begin{array}{l}\text { Humanitarian Emergency } \\
\text { Response Africa }\end{array}$ & $\begin{array}{l}\text { COVID-19 subnational } \\
\text { cases in Africa }\end{array}$ & $\begin{array}{l}\text { Reporting COVID- } \\
19 \text { cases on a } \\
\text { national level }\end{array}$ & $\begin{array}{l}\text { https://data.humdata.org/dataset/africa-coronavirus- } \\
\text { covid-19-subnational-cases[213] }\end{array}$ & [213] \\
\hline $\begin{array}{l}\text { Qatar Computing Research } \\
\text { Institute }\end{array}$ & $\begin{array}{l}\text { Twitter data geographic } \\
\text { distribution of COVID-19 }\end{array}$ & $\begin{array}{l}\text { Geographical } \\
\text { distribution of } \\
\text { twitter users and } \\
\text { tweets regarding } \\
\text { COVID-19 } \\
\text { pandemic. }\end{array}$ & $\begin{array}{l}\text { https://data.humdata.org/dataset/covid-19-twitter-data- } \\
\text { geographic-distribution[214] }\end{array}$ & [214] \\
\hline ACAPS & $\begin{array}{l}\text { Secondary impacts of } \\
\text { Covid } 19 \text { on a global scale }\end{array}$ & $\begin{array}{l}\text { Aid Decision- } \\
\text { making on } \\
\text { addressing wider } \\
\text { effects of COVID- } \\
19\end{array}$ & $\begin{array}{l}\text { https://data.humdata.org/dataset/global-covid-19- } \\
\text { secondary-impacts.[215] }\end{array}$ & {$[215]$} \\
\hline $\begin{array}{l}\text { Humanitarian Emergency } \\
\text { Response Africa }\end{array}$ & $\begin{array}{l}\text { COVID-19 city level in } \\
\text { Burkina Faso }\end{array}$ & $\begin{array}{l}\text { Reporting Covid } \\
\text { data at a city level }\end{array}$ & $\begin{array}{l}\text { https://data.humdata.org/dataset/burkinafaso_covid19_cit } \\
\text { y-level[216] }\end{array}$ & [216] \\
\hline Hub Latin America & $\begin{array}{l}\text { The COVID-19 mortality } \\
\text { rate in Lima, Peru }\end{array}$ & $\begin{array}{l}\text { Reporting, analysis } \\
\text { of COVID-19 death } \\
\text { rates in Lima }\end{array}$ & $\begin{array}{l}\text { https://data.humdata.org/dataset/peru-covid19-mortality- } \\
\text { rate-in-lima[217] }\end{array}$ & [217] \\
\hline Infoculture & $\begin{array}{l}\text { COVID-19 cases in } \\
\text { Moscow }\end{array}$ & Statistics & $\begin{array}{l}\text { https://data.humdata.org/dataset/covid-19-cases-data-in- } \\
\text { moscow[218] }\end{array}$ & [218] \\
\hline HDX & $\begin{array}{l}\text { Social measures and } \\
\text { public health applied } \\
\text { during COVID-19 }\end{array}$ & $\begin{array}{l}\text { Analysis and } \\
\text { reporting. }\end{array}$ & $\begin{array}{l}\text { https://data.humdata.org/dataset/world-global-database- } \\
\text { of-public-health-and-social-measures-applied-during-the- } \\
\text { covid-19-pandemic[219] }\end{array}$ & [219] \\
\hline Mobile Accord, Inc [GeoPoll] & $\begin{array}{l}\text { Impact and perceptions of } \\
\text { Coronavirus in Sub- } \\
\text { Saharan African countries }\end{array}$ & $\begin{array}{l}\text { Analysis and } \\
\text { reporting }\end{array}$ & $\begin{array}{l}\text { https://data.humdata.org/dataset/covid-19-impacts- } \\
\text { africa[220] }\end{array}$ & [220] \\
\hline HDX & $\begin{array}{l}\text { Subnational COVID-19 } \\
\text { cases for Iraq }\end{array}$ & $\begin{array}{l}\text { Reporting Covid } \\
\text { data at National } \\
\text { level }\end{array}$ & $\begin{array}{l}\text { https://data.humdata.org/dataset/iraq-coronavirus-covid- } \\
\text { 19-subnational-cases[221] }\end{array}$ & {$[221]$} \\
\hline HDX & $\begin{array}{l}\text { Covid } 19 \text { related funding } \\
\text { from IATI }\end{array}$ & $\begin{array}{l}\text { Monitoring of } \\
\text { funding use in } \\
\text { fighting COVID-19 }\end{array}$ & $\begin{array}{l}\text { https://data.humdata.org/dataset/iati-covid19- } \\
\text { funding[222] }\end{array}$ & [222] \\
\hline HDX & $\begin{array}{l}\text { Gavi and World Bank } \\
\text { COVID-19 vaccine } \\
\text { funding }\end{array}$ & $\begin{array}{l}\text { Fund disbursement } \\
\text { and support for } \\
\text { COVID-19 }\end{array}$ & $\begin{array}{l}\text { https://data.humdata.org/dataset/world-bank-and-gavi- } \\
\text { vaccine-financing[223] }\end{array}$ & [223] \\
\hline Code for Venezuela & $\begin{array}{l}\text { Survey on COVID-19 } \\
\text { impact }\end{array}$ & $\begin{array}{l}\text { Data analysis and } \\
\text { interpretation }\end{array}$ & $\begin{array}{l}\text { https://data.humdata.org/dataset/open_one_time_covid_i } \\
\text { mpact[224] }\end{array}$ & [224] \\
\hline $\begin{array}{l}\text { Humanitarian Emergency } \\
\text { Response Africa }\end{array}$ & $\begin{array}{l}\text { COVID-19 cases in } \\
\text { Ethiopia }\end{array}$ & $\begin{array}{l}\text { Reporting cases at a } \\
\text { national level. }\end{array}$ & $\begin{array}{l}\text { https://data.humdata.org/dataset/ethiopia-covid19- } \\
\text { cases[225] }\end{array}$ & [225] \\
\hline World Bank Group & $\begin{array}{l}\text { High frequency indicators } \\
\text { for COVID-19 }\end{array}$ & $\begin{array}{l}\text { Data analysis and } \\
\text { interpretation }\end{array}$ & $\begin{array}{l}\text { https://data.humdata.org/dataset/covid-19-high- } \\
\text { frequency-indicators[226] }\end{array}$ & {$[226]$} \\
\hline
\end{tabular}




\begin{tabular}{|c|c|c|c|c|}
\hline OCHA FISS & $\begin{array}{l}\text { Global humanitarian } \\
\text { response plan COVID-19 } \\
\text { administrative boundaries } \\
\text { and population-statistics }\end{array}$ & $\begin{array}{l}\text { Reporting cases at a } \\
\text { national level. }\end{array}$ & $\begin{array}{l}\text { https://data.humdata.org/dataset/global-humanitarian- } \\
\text { response-plan-covid-19-administrative-boundaries-and- } \\
\text { population-statistics[227] }\end{array}$ & [227] \\
\hline INFORM & $\begin{array}{l}\text { Inform Risk Index for } \\
\text { COVID-19, Version 0.1.4 }\end{array}$ & $\begin{array}{l}\text { Support } \\
\text { prioritization of } \\
\text { preparedness and } \\
\text { early response } \\
\text { actions for the direct } \\
\text { impacts of the } \\
\text { pandemic and } \\
\text { identify countries } \\
\text { where secondary } \\
\text { effects are likely to } \\
\text { have the most } \\
\text { critical humanitarian } \\
\text { consequences. }\end{array}$ & $\begin{array}{l}\text { https://data.humdata.org/dataset/inform-covid-19-risk- } \\
\text { index-version-0-1-4[228] }\end{array}$ & {$[228]$} \\
\hline Safeture & $\begin{array}{l}\text { COVID-19 subnational } \\
\text { cases in Kazakhstan }\end{array}$ & $\begin{array}{l}\text { For data analysis } \\
\text { and interpretation }\end{array}$ & $\begin{array}{l}\text { https://data.humdata.org/dataset/kazakhstan-coronavirus- } \\
\text { covid-19-subnational-cases. [229] }\end{array}$ & [229] \\
\hline OCHA Philippines & $\begin{array}{l}\text { COVID-19-operational } \\
\text { presence risk } \\
\text { communication and } \\
\text { community engagement in } \\
\text { the Philippines }\end{array}$ & $\begin{array}{l}\text { Risk communication } \\
\text { and community } \\
\text { engagement }\end{array}$ & $\begin{array}{l}\text { https://data.humdata.org/dataset/philippines-covid-19- } \\
\text { operational-presence-risk-communication-and- } \\
\text { community-engagement-rcce. [230] }\end{array}$ & {$[230]$} \\
\hline Hub Latin America & $\begin{array}{l}\text { Epidemiological and } \\
\text { hospital indicators on } \\
\text { COVID-19 in Ouro Preto, } \\
\text { Brazil }\end{array}$ & $\begin{array}{l}\text { For data analysis } \\
\text { and interpretation }\end{array}$ & $\begin{array}{l}\text { https://data.humdata.org/dataset/brazil-epidemiological- } \\
\text { and-hospital-indicators-on-covid-19-in-ouro-preto. [231] }\end{array}$ & {$[231]$} \\
\hline Safeture & $\begin{array}{l}\text { COVID-19 subnational } \\
\text { cases in Oman }\end{array}$ & $\begin{array}{l}\text { Reporting cases at a } \\
\text { national level. }\end{array}$ & $\begin{array}{l}\text { https://data.humdata.org/dataset/oman-coronavirus-covid- } \\
\text { 19-subnational-cases. [232] }\end{array}$ & {$[232]$} \\
\hline $\begin{array}{l}\text { Humanitarian Emergency } \\
\text { Response Africa }\end{array}$ & $\begin{array}{l}\text { Coronavirus [COVID-19] } \\
\text { City level cases for } \\
\text { Mauritania }\end{array}$ & $\begin{array}{l}\text { Reporting cases at a } \\
\text { national level. }\end{array}$ & $\begin{array}{l}\text { https://data.humdata.org/dataset/mauritania-coronavirus- } \\
\text { covid-19-city-level. [233] }\end{array}$ & [233] \\
\hline $\begin{array}{l}\text { UNICEF Data and Analytics } \\
\text { [HQ] }\end{array}$ & $\begin{array}{l}\text { Tracking children's } \\
\text { situation during COVID- } \\
19\end{array}$ & $\begin{array}{l}\text { Data analysis and } \\
\text { interpretation }\end{array}$ & $\begin{array}{l}\text { https://data.humdata.org/dataset/rapid-situation-tracking- } \\
\text { for-covid-19-socioeconomic-impacts. [234] }\end{array}$ & {$[234]$} \\
\hline $\begin{array}{l}\text { Humanitarian Emergency } \\
\text { Response Africa }\end{array}$ & $\begin{array}{l}\text { COVID-19 recoveries in } \\
\text { Africa on a national level }\end{array}$ & $\begin{array}{l}\text { Data analysis and } \\
\text { interpretation }\end{array}$ & $\begin{array}{l}\text { https://data.humdata.org/dataset/africa-covid-19- } \\
\text { recovered-cases. [235] }\end{array}$ & [235] \\
\hline Mobile Accord, Inc. [GeoPoll] & $\begin{array}{l}\text { COVID-19 vaccines and } \\
\text { impacts accepted in Sub- } \\
\text { Saharan Africa }\end{array}$ & $\begin{array}{l}\text { Data analysis and } \\
\text { interpretation }\end{array}$ & $\begin{array}{l}\text { https://data.humdata.org/dataset/covid19-impacts-and- } \\
\text { vaccine-acceptance-in-sub-saharan-africa. [236] }\end{array}$ & {$[236]$} \\
\hline $\begin{array}{l}\text { United Nations Development } \\
\text { Coordination Office }\end{array}$ & $\begin{array}{l}\text { UN Collective Results on } \\
\text { the COVID-19 } \\
\text { Socioeconomic Response } \\
\text { in } 2020\end{array}$ & $\begin{array}{l}\text { Monitor the progress } \\
\text { and achievements of } \\
\text { UNCT's collective } \\
\text { actions in socio- } \\
\text { economic response. }\end{array}$ & $\begin{array}{l}\text { https://data.humdata.org/dataset/un-collective-results-on- } \\
\text { the-covid-19-socioeconomic-response-in-2020. [237] }\end{array}$ & [237] \\
\hline Mobile Accord, Inc. [GeoPoll] & $\begin{array}{l}\text { Economic impact of } \\
\text { COVID-19 in Sub Saharan } \\
\text { Africa }\end{array}$ & $\begin{array}{l}\text { Data interpretation } \\
\text { and analysis }\end{array}$ & $\begin{array}{l}\text { https://data.humdata.org/dataset/economic-impact-of- } \\
\text { covid-19-in-sub-saharan-africa. [238] }\end{array}$ & [238] \\
\hline HDX & $\begin{array}{l}\text { COVID-19 subnational } \\
\text { cases in Myanmar }\end{array}$ & $\begin{array}{l}\text { Reporting cases at a } \\
\text { national level. }\end{array}$ & $\begin{array}{l}\text { https://data.humdata.org/dataset/myanmar-coronavirus- } \\
\text { covid-19-subnational-cases. [239] }\end{array}$ & [239] \\
\hline Safeture & $\begin{array}{l}\text { COVID-19 sub-national } \\
\text { cases in Ghana }\end{array}$ & $\begin{array}{l}\text { Reporting cases at a } \\
\text { national level. }\end{array}$ & $\begin{array}{l}\text { https://data.humdata.org/dataset/ghana-coronavirus- } \\
\text { covid-19-subnational-cases [240] }\end{array}$ & [240] \\
\hline Insecurity Insight & Covid 19 and Aid Security & $\begin{array}{l}\text { To help aid agencies } \\
\text { meet the duty of } \\
\text { care obligations to } \\
\text { staff and reach } \\
\text { people in need. }\end{array}$ & $\begin{array}{l}\text { https://data.humdata.org/dataset/aid-security-and-covid- } \\
\text { 19. [241] }\end{array}$ & {$[241]$} \\
\hline Infoculture & $\begin{array}{l}\text { Registry of Russian } \\
\text { NGO's affected by } \\
\text { COVID-19 }\end{array}$ & $\begin{array}{l}\text { For data analysis } \\
\text { and interpretation. }\end{array}$ & $\begin{array}{l}\text { https://data.humdata.org/dataset/ngos-affected-by- } \\
\text { covid19-russia. [242] }\end{array}$ & $\begin{array}{l}{[242][24} \\
3]\end{array}$ \\
\hline
\end{tabular}




\begin{tabular}{|c|c|c|c|c|}
\hline HDX & $\begin{array}{l}\text { Facility Interim } \\
\text { Distribution Forecast for } \\
\text { Covax }\end{array}$ & $\begin{array}{l}\text { For data analysis } \\
\text { and interpretation. }\end{array}$ & $\begin{array}{l}\text { https://data.humdata.org/dataset/covax-facility-interim- } \\
\text { distribution-forecast. [244] }\end{array}$ & {$[244]$} \\
\hline $\begin{array}{l}\text { UNHCR - The UN Refugee } \\
\text { Agency }\end{array}$ & $\begin{array}{l}\text { Socio-economic impact of } \\
\text { COVID-19 on refugees in } \\
\text { Kenya }\end{array}$ & $\begin{array}{l}\text { For data analysis } \\
\text { and interpretation. }\end{array}$ & $\begin{array}{l}\text { https://data.humdata.org/dataset/unhcr-ken-2020- } \\
\text { socioeconomic-impact-of-covid-19-on-pocs-in-kenya-v2- } \\
\text { 2. [245] }\end{array}$ & {$[245]$} \\
\hline $\begin{array}{l}\text { Humanitarian Emergency } \\
\text { Response Africa }\end{array}$ & $\begin{array}{l}\text { COVID-19 city level cases } \\
\text { in Togo }\end{array}$ & $\begin{array}{l}\text { Reporting cases at a } \\
\text { national level. }\end{array}$ & $\begin{array}{l}\text { https://data.humdata.org/dataset/togo-coronavirus-covid- } \\
\text { 19-city-level. [246] }\end{array}$ & {$[246]$} \\
\hline UNICEF Data and Analytics & $\begin{array}{l}\text { Indicators of interest to } \\
\text { COVID-19 data at } \\
\text { UNICEF }\end{array}$ & $\begin{array}{l}\text { For data analysis } \\
\text { and interpretation. }\end{array}$ & $\begin{array}{l}\text { https://data.humdata.org/dataset/unicef-indicators-of- } \\
\text { interest-to-the-covid-19-outbreak. [247] }\end{array}$ & {$[247]$} \\
\hline HDX & $\begin{array}{l}\text { COVID-19 subnational } \\
\text { cases in Mozambique }\end{array}$ & $\begin{array}{l}\text { Reporting cases at a } \\
\text { national level. }\end{array}$ & $\begin{array}{l}\text { https://data.humdata.org/dataset/mozambique- } \\
\text { coronavirus-covid-19-subnational-cases. [248] }\end{array}$ & {$[248]$} \\
\hline HDX & $\begin{array}{l}\text { COVID-19 subnational } \\
\text { cases for Haiti }\end{array}$ & $\begin{array}{l}\text { Reporting cases at a } \\
\text { national level. }\end{array}$ & $\begin{array}{l}\text { https://data.humdata.org/dataset/haiti-covid-19- } \\
\text { subnational-cases [249] }\end{array}$ & {$[249]$} \\
\hline OCHA HQ & $\begin{array}{l}\text { COVID-19 Pandemic } \\
\text { induced Humanitarian } \\
\text { Access Constraints }\end{array}$ & $\begin{array}{l}\text { For data analysis } \\
\text { and interpretation. }\end{array}$ & $\begin{array}{l}\text { https://data.humdata.org/dataset/constraints-faced-by- } \\
\text { people-due-to-covid-19-outbreak. [250] }\end{array}$ & {$[250]$} \\
\hline OCHA Philippines & $\begin{array}{l}2020 \text { Significant events } \\
\text { happening in Philippines }\end{array}$ & $\begin{array}{l}\text { For data analysis } \\
\text { and interpretation. }\end{array}$ & $\begin{array}{l}\text { https://data.humdata.org/dataset/philippines-2020- } \\
\text { significant-events. [251] }\end{array}$ & {$[251]$} \\
\hline $\begin{array}{l}\text { UNHCR - The UN Refugee } \\
\text { Agency }\end{array}$ & $\begin{array}{l}\text { Socio-economic impact of } \\
\text { COVID-19 on refugees in } \\
\text { Kenya round } 5\end{array}$ & $\begin{array}{l}\text { For data analysis } \\
\text { and interpretation. }\end{array}$ & $\begin{array}{l}\text { https://data.humdata.org/dataset/unhcr-ken-2020-covid- } \\
\text { round5-v2-1. [252] }\end{array}$ & $\begin{array}{l}{[252][25} \\
3]\end{array}$ \\
\hline OCHA Sudan & $\begin{array}{l}\text { COVID-19 response and } \\
\text { preparedness } 4 \mathrm{~W} \text { in Sudan }\end{array}$ & $\begin{array}{l}\text { COVID-19 response } \\
\text { outcomes }\end{array}$ & $\begin{array}{l}\text { https://data.humdata.org/dataset/sudan-covid-19- } \\
\text { preparedness-and-response-4w. [254] }\end{array}$ & {$[254]$} \\
\hline Indonesian Red Cross [PMI] & $\begin{array}{l}\text { Community Feedback by } \\
\text { Indonesian Red Cross } \\
\text { [PMI] }\end{array}$ & $\begin{array}{l}\text { COVID-19 response } \\
\text { outcomes }\end{array}$ & $\begin{array}{l}\text { https://data.humdata.org/dataset/community-feedback-by- } \\
\text { indonesian-red-cross-pmi. [255] }\end{array}$ & {$[255]$} \\
\hline $\begin{array}{l}\text { Johns Hopkins Applied Physics } \\
\text { Lab }\end{array}$ & $\begin{array}{l}\text { Projected COVID-19 sub- } \\
\text { national cases in Sudan }\end{array}$ & $\begin{array}{l}\text { For data analysis } \\
\text { and interpretation }\end{array}$ & $\begin{array}{l}\text { https://data.humdata.org/dataset/sudan-projected-covid- } \\
\text { 19-sub-national-cases. [256] }\end{array}$ & {$[256]$} \\
\hline $\begin{array}{l}\text { International Organization for } \\
\text { Migration }\end{array}$ & $\begin{array}{l}\text { IATA travel restriction } \\
\text { monitoring }\end{array}$ & $\begin{array}{l}\text { For data analysis } \\
\text { and interpretation. }\end{array}$ & $\begin{array}{l}\text { https://data.humdata.org/dataset/travel-restriction- } \\
\text { monitoring-iata-covid-19-iom-dtm. [257] }\end{array}$ & {$[257]$} \\
\hline OCHA ROWCA & $\begin{array}{l}\text { COVID-19 situation in } \\
\text { West and Central Africa }\end{array}$ & $\begin{array}{l}\text { For data analysis } \\
\text { and interpretation. }\end{array}$ & $\begin{array}{l}\text { https://data.humdata.org/dataset/west-and-central-africa- } \\
\text { coronavirus-covid-19-situation. [258] }\end{array}$ & {$[258]$} \\
\hline $\begin{array}{l}\text { Johns Hopkins Applied Physics } \\
\text { Lab }\end{array}$ & $\begin{array}{l}\text { Projected COVID-19 sub- } \\
\text { national cases for Somalia }\end{array}$ & $\begin{array}{l}\text { For data analysis } \\
\text { and interpretation. }\end{array}$ & $\begin{array}{l}\text { https://data.humdata.org/dataset/somalia-projected-covid- } \\
\text { 19-sub-national-cases [259] }\end{array}$ & [259] \\
\hline OCHA Ethiopia & $\begin{array}{l}\text { COVID-19 sub-national } \\
\text { cases for Ethiopia. }\end{array}$ & $\begin{array}{l}\text { Reporting cases at a } \\
\text { national level. }\end{array}$ & $\begin{array}{l}\text { https://data.humdata.org/dataset/ethiopia-coronavirus- } \\
\text { covid-19-subnational-cases [260] }\end{array}$ & {$[260]$} \\
\hline Infoculture & COVID-19 cases in Russia & $\begin{array}{l}\text { Reporting cases at a } \\
\text { national level. }\end{array}$ & $\begin{array}{l}\text { https://data.humdata.org/dataset/covid-19-cases-data-in- } \\
\text { russia. [261] }\end{array}$ & {$[261]$} \\
\hline Mobile Accord, Inc. [GeoPoll] & $\begin{array}{l}\text { Ongoing impacts of } \\
\text { COVID-19 in Sub-Saharan } \\
\text { Africa }\end{array}$ & $\begin{array}{l}\text { For data analysis } \\
\text { and interpretation. }\end{array}$ & $\begin{array}{l}\text { https://data.humdata.org/dataset/ongoing-impacts-of- } \\
\text { covid-19-in-sub-saharan-africa. [262] }\end{array}$ & {$[262]$} \\
\hline OCHA HQ & $\begin{array}{l}\text { Global appeals and plans } \\
\text { of COVID-19 around the } \\
\text { globe }\end{array}$ & $\begin{array}{l}\text { For data analysis } \\
\text { and interpretation. }\end{array}$ & $\begin{array}{l}\text { https://data.humdata.org/dataset/covid-19-global-appeals- } \\
\text { and-plans. [263] }\end{array}$ & {$[263]$} \\
\hline Mobile Accord, Inc. [GeoPoll] & $\begin{array}{l}\text { Community perception } \\
\text { and knowledge of Covid } \\
19 \text { in sub-Saharan Africa }\end{array}$ & $\begin{array}{l}\text { For data analysis } \\
\text { and interpretation. }\end{array}$ & $\begin{array}{l}\text { https://data.humdata.org/dataset/coronavirus-in-sub- } \\
\text { saharan-africa [264] }\end{array}$ & [264] \\
\hline OCHA HQ & $\begin{array}{l}\text { COVID-19 allocations for } \\
\text { CERF and CBPF }\end{array}$ & $\begin{array}{l}\text { Monitoring resource } \\
\text { allocation }\end{array}$ & $\begin{array}{l}\text { https://data.humdata.org/dataset/cerf-covid-19- } \\
\text { allocations. [265] }\end{array}$ & {$[265]$} \\
\hline INFORM & $\begin{array}{l}\text { INFORM COVID-19 } \\
\text { comparability and analysis } \\
\text { tool }\end{array}$ & $\begin{array}{l}\text { Identification of } \\
\text { countries at risk } \\
\text { from health and }\end{array}$ & $\begin{array}{l}\text { https://data.humdata.org/dataset/inform-covid-analysis- } \\
\text { v01. [266] }\end{array}$ & {$[266]$} \\
\hline
\end{tabular}




\begin{tabular}{|c|c|c|c|c|}
\hline & & $\begin{array}{l}\text { humanitarian } \\
\text { impacts of COVID- } \\
19 \text { that could } \\
\text { overwhelm current } \\
\text { national response } \\
\text { capacity, and } \\
\text { therefore lead to a } \\
\text { need for additional } \\
\text { international } \\
\text { assistance }\end{array}$ & & \\
\hline OCHA HQ & $\begin{array}{l}\text { Covid } 19 \text { impacts, } \\
\text { mitigation, and } \\
\text { humanitarian access } \\
\text { constraint. }\end{array}$ & $\begin{array}{l}\text { For data analysis } \\
\text { and interpretation. }\end{array}$ & $\begin{array}{l}\text { https://data.humdata.org/dataset/covid19-humanitarian- } \\
\text { access. [267] }\end{array}$ & [267] \\
\hline HDX & $\begin{array}{l}\text { LSHTM COVID-19 } \\
\text { Projections. }\end{array}$ & $\begin{array}{l}\text { For data analysis } \\
\text { and interpretation. }\end{array}$ & $\begin{array}{l}\text { https://data.humdata.org/dataset/lshtm-covid-19- } \\
\text { projections [268] }\end{array}$ & [268] \\
\hline UNICEF ESARO & $\begin{array}{l}\text { UNICEF COVID-19 } \\
\text { response and situation in } \\
\text { Eastern and Southern } \\
\text { Africa }\end{array}$ & $\begin{array}{l}\text { For data analysis } \\
\text { and interpretation. }\end{array}$ & $\begin{array}{l}\text { https://data.humdata.org/dataset/eastern-and-southern- } \\
\text { africa-covid-19-unicef-situation-and-response [269] }\end{array}$ & [269] \\
\hline OCHA Mali & $\begin{array}{l}\text { COVID-19 subnational } \\
\text { cases in Mali }\end{array}$ & $\begin{array}{l}\text { Reporting cases at a } \\
\text { national level. }\end{array}$ & $\begin{array}{l}\text { https://data.humdata.org/dataset/mali-coronavirus-covid- } \\
\text { 19-subnational-cases. [270] }\end{array}$ & {$[270]$} \\
\hline OCHA HQ & $\begin{array}{l}\text { Economic exposure index } \\
\text { for COVID-19 }\end{array}$ & $\begin{array}{l}\text { For data analysis } \\
\text { and interpretation. }\end{array}$ & $\begin{array}{l}\text { https://data.humdata.org/dataset/covid-19-economic- } \\
\text { exposure-index. [271] }\end{array}$ & {$[271]$} \\
\hline OCHA Somalia & $\begin{array}{l}\text { COVID-19 sub-national } \\
\text { cases for Somalia }\end{array}$ & $\begin{array}{l}\text { Reporting cases at a } \\
\text { national level. }\end{array}$ & $\begin{array}{l}\text { https://data.humdata.org/dataset/somalia-coronavirus- } \\
\text { covid-19-subnational-cases. [272] }\end{array}$ & $\begin{array}{l}{[272][27} \\
3]\end{array}$ \\
\hline $\begin{array}{l}\text { Johns Hopkins Applied Physics } \\
\text { Lab }\end{array}$ & $\begin{array}{l}\text { Projected COVID-19 sub- } \\
\text { national cases for } \\
\text { Afghanistan }\end{array}$ & $\begin{array}{l}\text { Reporting cases at a } \\
\text { national level. }\end{array}$ & $\begin{array}{l}\text { https://data.humdata.org/dataset/afghanistan-projected- } \\
\text { covid-19-sub-national-cases. [275] }\end{array}$ & $\begin{array}{l}{[274][27} \\
5]\end{array}$ \\
\hline $\begin{array}{l}\text { UNHCR - The UN Refugee } \\
\text { Agency }\end{array}$ & $\begin{array}{l}\text { Testing, knowledge, and } \\
\text { mask-wearing }\end{array}$ & $\begin{array}{l}\text { For data analysis } \\
\text { and interpretation. }\end{array}$ & $\begin{array}{l}\text { https://data.humdata.org/dataset/unhcr-bgd-2020-covid- } \\
\text { mwtk-v2-1. [276] }\end{array}$ & {$[276]$} \\
\hline Uganda Red Cross Society & COVID-19 risk index & $\begin{array}{l}\text { For data analysis } \\
\text { and interpretation. }\end{array}$ & $\begin{array}{l}\text { https://data.humdata.org/dataset/covid19_risk_index-zip. } \\
\text { [277] }\end{array}$ & {$[277]$} \\
\hline $\mathrm{HDX}$ & $\begin{array}{l}\text { COVID-19 subnational } \\
\text { cases for the Democratic } \\
\text { Republic of Congo }\end{array}$ & $\begin{array}{l}\text { Reporting cases at a } \\
\text { national level. }\end{array}$ & $\begin{array}{l}\text { https://data.humdata.org/dataset/democratic-republic-of- } \\
\text { the-congo-coronavirus-covid-19-subnational-cases. [278] }\end{array}$ & [278] \\
\hline HDX & $\begin{array}{l}\text { COVID-19 subnational } \\
\text { data for Libya }\end{array}$ & $\begin{array}{l}\text { Reporting cases at a } \\
\text { national level. }\end{array}$ & $\begin{array}{l}\text { https://data.humdata.org/dataset/libya-coronavirus-covid- } \\
\text { 19-subnational-cases. [279] }\end{array}$ & {$[279]$} \\
\hline $\begin{array}{l}\text { UNHCR - The UN Refugee } \\
\text { Agency }\end{array}$ & $\begin{array}{l}\text { Round } 2 \text { Socio-economic } \\
\text { impacts of COVID-19 on } \\
\text { refugees in Kenya }\end{array}$ & $\begin{array}{l}\text { For data analysis } \\
\text { and interpretation. }\end{array}$ & $\begin{array}{l}\text { https://data.humdata.org/dataset/unhcr-ken-2020- } \\
\text { socioeconomic-impact-of-covid-19-on-pocs-in-kenya- } \\
\text { round2-v1-0. [280] }\end{array}$ & [280] \\
\hline $\begin{array}{l}\text { International Organization for } \\
\text { Migration }\end{array}$ & $\begin{array}{l}\text { Cameroon COVID-19 } \\
\text { Mobility Restriction - } \\
\text { Point of Entries - [IOM } \\
\text { DTM] }\end{array}$ & $\begin{array}{l}\text { For data analysis } \\
\text { and interpretation. }\end{array}$ & $\begin{array}{l}\text { https://data.humdata.org/dataset/cameroon-covid-19- } \\
\text { mobility-restriction-point-of-entries-iom-dtm. [281] }\end{array}$ & {$[281]$} \\
\hline $\begin{array}{l}\text { UNHCR - The UN Refugee } \\
\text { Agency }\end{array}$ & $\begin{array}{l}\text { A panel study of the socio- } \\
\text { economic impacts of } \\
\text { COVID-19 on refugees } \\
\text { living in Kenya }\end{array}$ & $\begin{array}{l}\text { For data analysis } \\
\text { and interpretation. }\end{array}$ & $\begin{array}{l}\text { https://data.humdata.org/dataset/unhcr-ken-2020-covid- } \\
\text { panel-v2-1. [282] }\end{array}$ & {$[282]$} \\
\hline $\begin{array}{l}\text { Johns Hopkins Applied Physics } \\
\text { Lab }\end{array}$ & $\begin{array}{l}\text { Projected COVID-19 sub- } \\
\text { national cases for Iraq }\end{array}$ & $\begin{array}{l}\text { Reporting cases at a } \\
\text { national level. }\end{array}$ & $\begin{array}{l}\text { https://data.humdata.org/dataset/iraq-projected-covid-19- } \\
\text { sub-national-cases. [282] }\end{array}$ & [283] \\
\hline $\begin{array}{l}\text { UNHCR - The UN Refugee } \\
\text { Agency }\end{array}$ & $\begin{array}{l}\text { Assessment of COVID-19 } \\
\text { socio-economic impacts } \\
\text { on Persons of concern to } \\
\text { UNHCR }\end{array}$ & $\begin{array}{l}\text { Reporting cases at } \\
\text { national level. }\end{array}$ & $\begin{array}{l}\text { https://data.humdata.org/dataset/unhcr-nga-2020-sea- } \\
\text { covid19-v2-1. [284] }\end{array}$ & $\begin{array}{l}{[284][28} \\
5]\end{array}$ \\
\hline $\begin{array}{l}\text { UNHCR - The UN Refugee } \\
\text { Agency }\end{array}$ & $\begin{array}{l}\text { Assessment of COVID-19 } \\
\text { impact on livelihoods of } \\
\text { refugees in Zambia }\end{array}$ & $\begin{array}{l}\text { For data analysis } \\
\text { and interpretation. }\end{array}$ & $\begin{array}{l}\text { https://data.humdata.org/dataset/ddi-zam-unhcr-covid19- } \\
\text { impact-assessment-on-refugee-livelihoods-zambia-july- } \\
\text { 2020. [286] }\end{array}$ & {$[286]$} \\
\hline
\end{tabular}




\begin{tabular}{|c|c|c|c|c|}
\hline Hub Latin America & $\begin{array}{l}\text { symptomatology related to } \\
\text { the coronavirus COVID- } \\
19 \text { in Ecuador }\end{array}$ & $\begin{array}{l}\text { Data exploration, } \\
\text { analysis }\end{array}$ & $\begin{array}{l}\text { https://data.humdata.org/dataset/symptomatology- } \\
\text { ecu911-santa-cruz-monthly-2018-2021. [287] }\end{array}$ & $\begin{array}{l}{[287][28} \\
8]\end{array}$ \\
\hline HDX & $\begin{array}{l}\text { Health facilities by } \\
\text { province in Afghanistan }\end{array}$ & $\begin{array}{l}\text { For data analysis } \\
\text { and interpretation. }\end{array}$ & $\begin{array}{l}\text { https://data.humdata.org/dataset/afghanistan-covid-19- } \\
\text { health-facilities-by-province. [289] }\end{array}$ & [289] \\
\hline ACAPS & $\begin{array}{l}\text { COVID-19 humanitarian } \\
\text { exceptions }\end{array}$ & $\begin{array}{l}\text { For data analysis } \\
\text { and interpretation. }\end{array}$ & $\begin{array}{l}\text { https://data.humdata.org/dataset/acaps-covid-19- } \\
\text { humanitarian-exemptions-dataset [290] }\end{array}$ & [290] \\
\hline $\begin{array}{l}\text { International Organization for } \\
\text { Migration }\end{array}$ & $\begin{array}{l}\text { COVID-19 mobility and } \\
\text { preparedness updates in } \\
\text { South Sudan. }\end{array}$ & $\begin{array}{l}\text { For data analysis } \\
\text { and interpretation. }\end{array}$ & $\begin{array}{l}\text { https://data.humdata.org/dataset/south-sudan-covid-19- } \\
\text { mobility-and-preparedness-updates-iom-dtm. [291] }\end{array}$ & [291] \\
\hline $\begin{array}{l}\text { UNHCR - The UN Refugee } \\
\text { Agency }\end{array}$ & $\begin{array}{l}\text { Socio-economic impacts } \\
\text { of COVID-19 on refugees } \\
\text { living in Kenya, Round } 1\end{array}$ & $\begin{array}{l}\text { For data analysis } \\
\text { and interpretation. }\end{array}$ & $\begin{array}{l}\text { https://data.humdata.org/dataset/unhcr-ken-2020-covid- } \\
\text { round1-v2-2. [292] }\end{array}$ & [292] \\
\hline $\begin{array}{l}\text { UNHCR - The UN Refugee } \\
\text { Agency }\end{array}$ & $\begin{array}{l}\text { Socio-economic impacts } \\
\text { of COVID-19 on refugees } \\
\text { living in Kenya, Round } 4\end{array}$ & $\begin{array}{l}\text { For data analysis } \\
\text { and interpretation. }\end{array}$ & $\begin{array}{l}\text { https://data.humdata.org/dataset/unhcr-ken-2020-covid- } \\
\text { round4-v2-1. [293] }\end{array}$ & [293] \\
\hline $\begin{array}{l}\text { UNHCR - The UN Refugee } \\
\text { Agency }\end{array}$ & $\begin{array}{l}\text { Socio-economic impacts } \\
\text { of COVID-19 on refugees } \\
\text { living in Kenya, Round } 3\end{array}$ & $\begin{array}{l}\text { For data analysis } \\
\text { and interpretation. }\end{array}$ & $\begin{array}{l}\text { https://data.humdata.org/dataset/unhcr-ken-2020-covid- } \\
\text { round3-v2-1. [294] }\end{array}$ & [294] \\
\hline \multirow[t]{10}{*}{$\begin{array}{l}\text { European Centre for Disease } \\
\text { Prevention and Control }\end{array}$} & $\begin{array}{l}\text { COVID-19 vaccination in } \\
\text { the EU/EEA }\end{array}$ & $\begin{array}{l}\text { Vaccine } \\
\text { administration } \\
\text { updates }\end{array}$ & $\begin{array}{l}\text { https://www.ecdc.europa.eu/en/publications-data/data- } \\
\text { COVID-19-vaccination-eu-eea. [295] }\end{array}$ & [295] \\
\hline & $\begin{array}{l}\text { Data on the daily number } \\
\text { of new reported COVID- } \\
19 \text { cases and deaths by } \\
\text { EU/EEA country }\end{array}$ & $\begin{array}{l}\text { Monitoring daily } \\
\text { COVID-19 cases. }\end{array}$ & $\begin{array}{l}\text { https://www.ecdc.europa.eu/en/publications-data/data- } \\
\text { daily-new-cases-COVID-19-eueea-country. [296] }\end{array}$ & [296] \\
\hline & $\begin{array}{l}\text { Data on SARS-CoV-2 } \\
\text { variants in the EU/EEA }\end{array}$ & $\begin{array}{l}\text { Monitoring SARS- } \\
\text { CoV-2 variants in } \\
\text { the EU/EEA }\end{array}$ & $\begin{array}{l}\text { https://www.ecdc.europa.eu/en/publications-data/data- } \\
\text { virus-variants-COVID-19-eueea. [297] }\end{array}$ & [297] \\
\hline & $\begin{array}{l}\text { Data on 14-day } \\
\text { notification rate of new } \\
\text { COVID-19 cases and } \\
\text { deaths }\end{array}$ & $\begin{array}{l}\text { Monitoring and } \\
\text { analysis of Data on } \\
\text { 14-day notification } \\
\text { rate of new COVID- } \\
19 \text { cases and deaths }\end{array}$ & $\begin{array}{l}\text { https://www.ecdc.europa.eu/en/publications-data/data- } \\
\text { national-14-day-notification-rate-COVID-19. [298] }\end{array}$ & $\begin{array}{l}{[298][29} \\
9]\end{array}$ \\
\hline & $\begin{array}{l}\text { Data on the daily } \\
\text { subnational 14-day } \\
\text { notification rate of new } \\
\text { COVID-19 cases }\end{array}$ & $\begin{array}{l}\text { Monitoring and } \\
\text { analysis. }\end{array}$ & $\begin{array}{l}\text { https://www.ecdc.europa.eu/en/publications- } \\
\text { data/subnational-14-day-notification-rate-COVID-19. } \\
\text { [300] }\end{array}$ & $\begin{array}{l}{[300][30} \\
1]\end{array}$ \\
\hline & $\begin{array}{l}\text { Data on hospital and ICU } \\
\text { admission rates and } \\
\text { current occupancy for } \\
\text { COVID-19 }\end{array}$ & $\begin{array}{l}\text { Monitoring and } \\
\text { analysis. }\end{array}$ & $\begin{array}{l}\text { https://www.ecdc.europa.eu/en/publications- } \\
\text { data/download-data-hospital-and-icu-admission-rates- } \\
\text { and-current-occupancy-COVID-19. [302] }\end{array}$ & $\begin{array}{l}{[302][30} \\
3]\end{array}$ \\
\hline & $\begin{array}{l}\text { Data on country response } \\
\text { measures }\end{array}$ & $\begin{array}{l}\text { Monitoring and } \\
\text { analysis. }\end{array}$ & $\begin{array}{l}\text { https://www.ecdc.europa.eu/en/publications- } \\
\text { data/download-data-response-measures-COVID-19 [304] }\end{array}$ & [304] \\
\hline & $\begin{array}{l}\text { Data on age-specific } \\
\text { notification rate }\end{array}$ & $\begin{array}{l}\text { Monitoring and } \\
\text { analysis. }\end{array}$ & $\begin{array}{l}\text { https://www.ecdc.europa.eu/en/publications- } \\
\text { data/COVID-19-data-14-day-age-notification-rate-new- } \\
\text { cases. [305] }\end{array}$ & [305] \\
\hline & $\begin{array}{l}\text { Data on council } \\
\text { recommendations for } \\
\text { mapping the coordinated } \\
\text { approach to the restriction } \\
\text { of free movement in } \\
\text { response to the COVID-19 } \\
\text { pandemic in the EU/EEA }\end{array}$ & $\begin{array}{l}\text { Monitoring and } \\
\text { analysis. }\end{array}$ & $\begin{array}{l}\text { https://www.ecdc.europa.eu/en/publications- } \\
\text { data/indicators-maps-support-council-recommendation. } \\
\text { [306] }\end{array}$ & [306] \\
\hline & $\begin{array}{l}\text { Historical data on the } \\
\text { COVID- } 19 \text { daily number } \\
\text { of cases and deaths by } \\
\text { country, worldwide }\end{array}$ & $\begin{array}{l}\text { Monitoring and } \\
\text { analysis. }\end{array}$ & $\begin{array}{l}\text { https://www.ecdc.europa.eu/en/publications- } \\
\text { data/download-todays-data-geographic-distribution- } \\
\text { COVID-19-cases-worldwide. [307] }\end{array}$ & [307] \\
\hline Kaggle.com & $\begin{array}{l}\text { Daily information on the } \\
\text { number of COVID-19 }\end{array}$ & $\begin{array}{l}\text { Monitoring and } \\
\text { analysis. }\end{array}$ & $\begin{array}{l}\text { https://www.kaggle.com/sudalairajkumar/novel-corona- } \\
\text { virus-2019-dataset [308] }\end{array}$ & [308] \\
\hline
\end{tabular}




\begin{tabular}{|c|c|c|c|c|}
\hline & $\begin{array}{l}\text { affected areas across the } \\
\text { globe }\end{array}$ & & & \\
\hline World Health Organization & $\begin{array}{l}\text { Information on country } \\
\text { reported public measures } \\
\text { to curb COVID-19. }\end{array}$ & $\begin{array}{l}\text { Monitoring and } \\
\text { analysis }\end{array}$ & $\begin{array}{l}\text { https://www.who.int/emergencies/diseases/novel- } \\
\text { coronavirus-2019/phsm. [309] }\end{array}$ & [309] \\
\hline $\begin{array}{l}\text { Johns Hopkins’ electronic } \\
\text { medical record, Epic }\end{array}$ & $\begin{array}{l}\text { Information on the patients } \\
\text { that have been confirmed } \\
\text { or are suspected of having } \\
\text { contracted COVID-19 }\end{array}$ & $\begin{array}{l}\text { For retrospective } \\
\text { analysis of COVID- } \\
19 \text { patient } \\
\text { populations }\end{array}$ & https://ictr.johnshopkins.edu/coronavirus/jh-crown/ [310] & [310] \\
\hline $\begin{array}{l}\text { National Patient-Centered } \\
\text { Clinical Research Network }\end{array}$ & $\begin{array}{l}\text { Data model tracking } \\
\text { insights on patients } \\
\text { infected with COVID-19 }\end{array}$ & $\begin{array}{l}\text { For understanding } \\
\text { and defining } \\
\text { demographics } \\
\text { infected with SARS- } \\
\text { CoV-2 }\end{array}$ & $\begin{array}{l}\text { https://pcornet.org/news/pcornet-COVID-19-common- } \\
\text { data-model-launched-enabling-rapid-capture-of- } \\
\text { insights/[311] }\end{array}$ & [311] \\
\hline $\begin{array}{l}\text { Johns Hopkins COVID-19 } \\
\text { collaboration platform }\end{array}$ & $\begin{array}{l}\text { Publicizing protocols } \\
\text { whose PIs are open to } \\
\text { various levels of } \\
\text { collaboration. }\end{array}$ & $\begin{array}{l}\text { Protocol } \\
\text { collaboration. }\end{array}$ & https://covidcp.org/. [312] & [312] \\
\hline $\begin{array}{l}\text { National COVID Cohort } \\
\text { Collaborative }\end{array}$ & $\begin{array}{l}\text { Building a centralized } \\
\text { national data resource that } \\
\text { the research community } \\
\text { can use to study COVID- } \\
19 \text { and identify potential } \\
\text { treatments as the pandemic } \\
\text { continues to evolve. }\end{array}$ & $\begin{array}{l}\text { Rapid collection and } \\
\text { analysis of clinical, } \\
\text { laboratory, and } \\
\text { diagnostic data from } \\
\text { hospitals and health } \\
\text { care plans }\end{array}$ & https://ncats.nih.gov/n3c/about. [313] & [313] \\
\hline 4CE & $\begin{array}{l}\text { COVID-19 positive cases } \\
\text { and new death rates by } \\
\text { country, overtime }\end{array}$ & $\begin{array}{l}\text { For data analysis } \\
\text { and interpretation }\end{array}$ & $\begin{array}{l}\text { https://covidclinical.net/plots/paper-01/release-2020-04- } \\
\text { 11/dailycounts.html. [314] }\end{array}$ & [314] \\
\hline 4CE & $\begin{array}{l}\text { COVID-19 number of } \\
\text { patients by country, by } \\
\text { gender }\end{array}$ & $\begin{array}{l}\text { For data analysis } \\
\text { and interpretation }\end{array}$ & $\begin{array}{l}\text { https://covidclinical.net/plots/paper-01/release-2020-04- } \\
\text { 11/demographics.html [315] }\end{array}$ & [315] \\
\hline 4CE & $\begin{array}{l}\text { COVID-19 lab values } \\
\text { corresponding to } 14 \\
\text { LOINC Codes }\end{array}$ & $\begin{array}{l}\text { For data analysis } \\
\text { and interpretation }\end{array}$ & $\begin{array}{l}\text { https://covidclinical.net/plots/paper-01/release-2020-04- } \\
\text { 11/labs.html [316] }\end{array}$ & [316] \\
\hline 4CE & $\begin{array}{l}\text { Comparison of data from } \\
\text { CSSE JHU }\end{array}$ & $\begin{array}{l}\text { For data analysis } \\
\text { and interpretation }\end{array}$ & $\begin{array}{l}\text { https:/covidclinical.net/plots/paper-01/release-2020-04- } \\
\text { 11/change.html. } \\
\text { [317] }\end{array}$ & [317] \\
\hline 4CE & $\begin{array}{l}\text { Participating sites } \\
\text { visualized on maps }\end{array}$ & $\begin{array}{l}\text { For data analysis } \\
\text { and interpretation }\end{array}$ & $\begin{array}{l}\text { https://covidclinical.net/plots/paper-01/release-2020-04- } \\
\text { 11/sites.html [318] }\end{array}$ & [318] \\
\hline 4CE & $\begin{array}{l}\text { Daily Count Data for } \\
\text { International Electronic } \\
\text { Health Record-Derived } \\
\text { COVID-19 Clinical } \\
\text { Course Profile }\end{array}$ & $\begin{array}{l}\text { For data analysis } \\
\text { and interpretation }\end{array}$ & $\begin{array}{l}\text { https://figshare.com/articles/dataset/Daily_Count_Data_f } \\
\text { or_International_Electronic_Health_Record- } \\
\text { Derived_COVID- } \\
\text { 19_Clinical_Course_Profile_The_4CE_Consortium/1215 } \\
\text { 2976/1. [319] }\end{array}$ & [319] \\
\hline 4CE & $\begin{array}{l}\text { Demographic data for } \\
\text { International Electronic } \\
\text { Health Record-Derived } \\
\text { COVID-19 Clinical } \\
\text { Course Profile. }\end{array}$ & $\begin{array}{l}\text { For data analysis } \\
\text { and interpretation }\end{array}$ & $\begin{array}{l}\text { https://figshare.com/articles/dataset/Demographics_Data_ } \\
\text { for_International_Electronic_Health_Record- } \\
\text { Derived_COVID- } \\
\text { 19_Clinical_Course_Profile_The_4CE_Consortium/1215 } \\
\text { 2973/1 [320] }\end{array}$ & [320] \\
\hline 4CE & $\begin{array}{l}\text { Diagnosis data for } \\
\text { International Electronic } \\
\text { Health Record-Derived } \\
\text { COVID-19 Clinical } \\
\text { Course Profile. }\end{array}$ & $\begin{array}{l}\text { For data analysis } \\
\text { and interpretation }\end{array}$ & $\begin{array}{l}\text { https://figshare.com/articles/dataset/Diagnosis_Data_for_ } \\
\text { International_Electronic_Health_Record- } \\
\text { Derived_COVID- } \\
\text { 19_Clinical_Course_Profile_The_4CE_Consortium/1215 } \\
2967 \text { [321] }\end{array}$ & [321] \\
\hline 4CE & $\begin{array}{l}\text { Labs data for International } \\
\text { Electronic Health Record- } \\
\text { Derived COVID-19 } \\
\text { Clinical Course Profile. }\end{array}$ & $\begin{array}{l}\text { For data analysis } \\
\text { and interpretation }\end{array}$ & $\begin{array}{l}\text { https://figshare.com/articles/dataset/Labs_Data_for_Inter } \\
\text { national_Electronic_Health_Record-Derived_COVID- } \\
\text { 19_Clinical_Course_Profile_The_4CE_Consortium/1215 } \\
\text { 2766 [322] }\end{array}$ & [322] \\
\hline 4CE & $\begin{array}{l}\text { Labs data for International } \\
\text { Electronic Health Record- } \\
\text { Derived COVID-19 }\end{array}$ & $\begin{array}{l}\text { For data analysis } \\
\text { and interpretation }\end{array}$ & $\begin{array}{l}\text { https://figshare.com/articles/dataset/Healthcare_Systems/ } \\
12118911 \text { [323] }\end{array}$ & [323] \\
\hline
\end{tabular}




\begin{tabular}{|c|c|c|c|c|}
\hline & Clinical Course Profile. & & & \\
\hline 4CE & $\begin{array}{l}\text { Time series COVID-19 } \\
\text { confirmed cases }\end{array}$ & $\begin{array}{l}\text { For data analysis } \\
\text { and interpretation }\end{array}$ & $\begin{array}{l}\text { https://github.com/CSSEGISandData/COVID- } \\
\text { 19/blob/dcd4181613f512a6f75249fc77b63286aebe7271/c } \\
\text { sse_covid_19_data/csse_covid_19_time_series/time_seri } \\
\text { es_covid19_confirmed_global.csv [324] }\end{array}$ & [324] \\
\hline Health and Retirement Study & $\begin{array}{l}2020 \text { HRS COVID-19 } \\
\text { project }\end{array}$ & $\begin{array}{l}\text { For data analysis } \\
\text { and interpretation }\end{array}$ & $\begin{array}{l}\text { https://hrsdata.isr.umich.edu/data-products/2020-hrs- } \\
\text { COVID-19-project. [325] }\end{array}$ & [325] \\
\hline COVID-19 research database & $\begin{array}{l}\text { Electronic health records, } \\
\text { claims, and consumer data. }\end{array}$ & $\begin{array}{l}\text { For data analysis } \\
\text { and interpretation }\end{array}$ & https://covid19researchdatabase.org/. [326] & [326] \\
\hline $\begin{array}{l}\text { COVID-19 Research Initiatives } \\
\text { in the HRS International Network }\end{array}$ & $\begin{array}{l}\text { HRS COVID-19 Data on } \\
\text { questionnaires, surveys, } \\
\text { interviews, and state } \\
\text { policies }\end{array}$ & $\begin{array}{l}\text { For data analysis } \\
\text { and interpretation }\end{array}$ & https://hrs.isr.umich.edu/data-products/COVID-19 [327] & [327] \\
\hline $\begin{array}{l}\text { Center for Disease Control and } \\
\text { Prevention }\end{array}$ & $\begin{array}{l}\text { COVID-19 Case } \\
\text { Surveillance Public Use } \\
\text { Data with Geography }\end{array}$ & $\begin{array}{l}\text { For analysis and } \\
\text { interpretation }\end{array}$ & $\begin{array}{l}\text { https://data.cdc.gov/Case-Surveillance/COVID-19-Case- } \\
\text { Surveillance-Public-Use-Data-with-Ge/n8mc-b4w4. } \\
\text { [328] }\end{array}$ & {$[328]$} \\
\hline $\begin{array}{l}\text { Center for Disease Control and } \\
\text { Prevention }\end{array}$ & $\begin{array}{l}\text { COVID-19 Case } \\
\text { Surveillance Public Use } \\
\text { Data }\end{array}$ & $\begin{array}{l}\text { For analysis and } \\
\text { interpretation }\end{array}$ & $\begin{array}{l}\text { https://data.cdc.gov/Case-Surveillance/COVID-19-Case- } \\
\text { Surveillance-Public-Use-Data/vbim-akqf. [329] }\end{array}$ & [329] \\
\hline $\begin{array}{l}\text { Center for Disease Control and } \\
\text { Prevention }\end{array}$ & $\begin{array}{l}\text { COVID-19 Case } \\
\text { Surveillance Restricted } \\
\text { Access Detailed Data }\end{array}$ & $\begin{array}{l}\text { For analysis and } \\
\text { interpretation }\end{array}$ & $\begin{array}{l}\text { https://data.cdc.gov/Case-Surveillance/COVID-19-Case- } \\
\text { Surveillance-Restricted-Access-Detai/mbd7-r32t. [330] }\end{array}$ & [330] \\
\hline $\begin{array}{l}\text { Center for Disease Control and } \\
\text { Prevention }\end{array}$ & $\begin{array}{l}\text { COVID-19 Vaccine } \\
\text { Distribution Allocations } \\
\text { by Jurisdiction - Janssen }\end{array}$ & $\begin{array}{l}\text { For analysis and } \\
\text { interpretation }\end{array}$ & $\begin{array}{l}\text { https://data.cdc.gov/Vaccinations/COVID-19-Vaccine- } \\
\text { Distribution-Allocations-by-Juris/w9zu-fywh [331] }\end{array}$ & [331] \\
\hline $\begin{array}{l}\text { Center for Disease Control and } \\
\text { Prevention }\end{array}$ & $\begin{array}{l}\text { COVID-19 Vaccine } \\
\text { Distribution Allocations } \\
\text { by Jurisdiction - Pfizer }\end{array}$ & $\begin{array}{l}\text { For analysis and } \\
\text { interpretation }\end{array}$ & $\begin{array}{l}\text { https://data.cdc.gov/Vaccinations/COVID-19-Vaccine- } \\
\text { Distribution-Allocations-by-Juris/saz5-9hgg [332] }\end{array}$ & $\begin{array}{l}{[332][33} \\
3]\end{array}$ \\
\hline $\begin{array}{l}\text { Center for Disease Control and } \\
\text { Prevention }\end{array}$ & $\begin{array}{l}\text { United States COVID-19 } \\
\text { Cases and Deaths by State } \\
\text { over Time }\end{array}$ & $\begin{array}{l}\text { For analysis and } \\
\text { interpretation }\end{array}$ & $\begin{array}{l}\text { https://data.cdc.gov/Case-Surveillance/United-States- } \\
\text { COVID-19-Cases-and-Deaths-by-State-o/9mfq-cb36 } \\
\text { [334] }\end{array}$ & [334] \\
\hline $\begin{array}{l}\text { Center for Disease Control and } \\
\text { Prevention }\end{array}$ & $\begin{array}{l}\text { COVID-19 Vaccine } \\
\text { Distribution Allocations } \\
\text { by Jurisdiction - Moderna }\end{array}$ & $\begin{array}{l}\text { For analysis and } \\
\text { interpretation }\end{array}$ & $\begin{array}{l}\text { https://data.cdc.gov/Vaccinations/COVID-19-Vaccine- } \\
\text { Distribution-Allocations-by-Juris/b7pe-5nws. [335] }\end{array}$ & $\begin{array}{l}{[335][33} \\
6]\end{array}$ \\
\hline $\begin{array}{l}\text { Center for Disease Control and } \\
\text { Prevention }\end{array}$ & $\begin{array}{l}\text { Provider Relief Fund } \\
\text { COVID-19 Nursing Home } \\
\text { Quality Incentive Program }\end{array}$ & $\begin{array}{l}\text { For analysis and } \\
\text { interpretation }\end{array}$ & $\begin{array}{l}\text { https://data.cdc.gov/Administrative/Provider-Relief- } \\
\text { Fund-COVID-19-Nursing-Home-Quality/bfqg-cb6d } \\
\text { [337] }\end{array}$ & {$[337]$} \\
\hline $\begin{array}{l}\text { Center for Disease Control and } \\
\text { Prevention }\end{array}$ & $\begin{array}{l}\text { Indicators of Anxiety or } \\
\text { Depression Based on } \\
\text { Reported Frequency of } \\
\text { Symptoms During Last } 7 \\
\text { Days }\end{array}$ & $\begin{array}{l}\text { For analysis and } \\
\text { interpretation }\end{array}$ & $\begin{array}{l}\text { https://data.cdc.gov/NCHS/Indicators-of-Anxiety-or- } \\
\text { Depression-Based-on-Repor/8pt5-q6wp [338] }\end{array}$ & [338] \\
\hline $\begin{array}{l}\text { Center for Disease Control and } \\
\text { Prevention }\end{array}$ & $\begin{array}{l}\text { Mental Health Care in the } \\
\text { Last } 4 \text { Weeks }\end{array}$ & $\begin{array}{l}\text { For analysis and } \\
\text { interpretation }\end{array}$ & $\begin{array}{l}\text { https://data.cdc.gov/NCHS/Mental-Health-Care-in-the- } \\
\text { Last-4-Weeks/yni7-er2q [339] }\end{array}$ & $\begin{array}{l}{[339][34} \\
0]\end{array}$ \\
\hline $\begin{array}{l}\text { Center for Disease Control and } \\
\text { Prevention }\end{array}$ & $\begin{array}{l}\text { Vaccine Hesitancy for } \\
\text { COVID-19: County and } \\
\text { local estimate }\end{array}$ & $\begin{array}{l}\text { For analysis and } \\
\text { interpretation }\end{array}$ & $\begin{array}{l}\text { https://data.cdc.gov/Vaccinations/Vaccine-Hesitancy-for- } \\
\text { COVID-19-County-and-local-es/q9mh-h2tw. [341] }\end{array}$ & {$[341]$} \\
\hline $\begin{array}{l}\text { Center for Disease Control and } \\
\text { Prevention }\end{array}$ & $\begin{array}{l}\text { Loss of Work Due to } \\
\text { Illness from COVID-19 }\end{array}$ & $\begin{array}{l}\text { For analysis and } \\
\text { interpretation }\end{array}$ & $\begin{array}{l}\text { https://data.cdc.gov/NCHS/Loss-of-Work-Due-to-Illness- } \\
\text { from-COVID-19/qgkx-mswu. [342] }\end{array}$ & [342] \\
\hline $\begin{array}{l}\text { Center for Disease Control and } \\
\text { Prevention }\end{array}$ & $\begin{array}{l}\text { COVID-19 Vaccinations } \\
\text { in the United States by } \\
\text { Jurisdiction }\end{array}$ & $\begin{array}{l}\text { For analysis and } \\
\text { interpretation }\end{array}$ & $\begin{array}{l}\text { https://data.cdc.gov/Vaccinations/COVID-19- } \\
\text { Vaccinations-in-the-United-States-Jurisdi/unsk-b7fc. } \\
\text { [343] }\end{array}$ & [343] \\
\hline $\begin{array}{l}\text { Center for Disease Control and } \\
\text { Prevention }\end{array}$ & $\begin{array}{l}\text { Provider Relief Fund \& } \\
\text { Accelerated and Advance } \\
\text { Payments }\end{array}$ & $\begin{array}{l}\text { For analysis and } \\
\text { interpretation }\end{array}$ & $\begin{array}{l}\text { https://data.cdc.gov/Administrative/Provider-Relief- } \\
\text { Fund-Accelerated-and-Advance-Payme/v2pi-w3up [344] }\end{array}$ & [344] \\
\hline $\begin{array}{l}\text { Center for Disease Control and } \\
\text { Prevention }\end{array}$ & $\begin{array}{l}\text { Indicators of Reduced } \\
\text { Access to Care Due to the } \\
\text { Coronavirus Pandemic } \\
\text { During Last } 4 \text { Weeks }\end{array}$ & $\begin{array}{l}\text { For analysis and } \\
\text { interpretation }\end{array}$ & $\begin{array}{l}\text { https://data.cdc.gov/NCHS/Indicators-of-Reduced- } \\
\text { Access-to-Care-Due-to-the-Co/xb3p-q62w. [345] }\end{array}$ & [345] \\
\hline
\end{tabular}




\begin{tabular}{|c|c|c|c|c|}
\hline $\begin{array}{l}\text { Center for Disease Control and } \\
\text { Prevention }\end{array}$ & $\begin{array}{l}\text { Access and Use of } \\
\text { Telemedicine During } \\
\text { COVID-19 }\end{array}$ & $\begin{array}{l}\text { For analysis and } \\
\text { interpretation }\end{array}$ & $\begin{array}{l}\text { https://data.cdc.gov/NCHS/Access-and-Use-of- } \\
\text { Telemedicine-During-COVID-19/8xy9-ubqz. [346] }\end{array}$ & $\begin{array}{l}{[346][34} \\
7]\end{array}$ \\
\hline $\begin{array}{l}\text { Center for Disease Control and } \\
\text { Prevention }\end{array}$ & $\begin{array}{l}\text { COVID-19 Vaccination } \\
\text { Trends in the United } \\
\text { States, National and } \\
\text { Jurisdictional data }\end{array}$ & $\begin{array}{l}\text { For analysis and } \\
\text { interpretation }\end{array}$ & $\begin{array}{l}\text { https://data.cdc.gov/Vaccinations/COVID-19- } \\
\text { Vaccination-Trends-in-the-United-States-N/rh2h-3yt2 } \\
\text { [348] }\end{array}$ & [348] \\
\hline $\begin{array}{l}\text { Center for Disease Control and } \\
\text { Prevention }\end{array}$ & $\begin{array}{l}\text { Reduced Access to Care } \\
\text { During COVID-19 }\end{array}$ & $\begin{array}{l}\text { For analysis and } \\
\text { interpretation }\end{array}$ & $\begin{array}{l}\text { https://data.cdc.gov/NCHS/Reduced-Access-to-Care- } \\
\text { During-COVID-19/th9n-ghnr. [349] }\end{array}$ & [349] \\
\hline $\begin{array}{l}\text { Center for Disease Control and } \\
\text { Prevention }\end{array}$ & $\begin{array}{l}\text { Telemedicine Use in the } \\
\text { Last } 4 \text { Weeks }\end{array}$ & $\begin{array}{l}\text { For analysis and } \\
\text { interpretation }\end{array}$ & $\begin{array}{l}\text { https://data.cdc.gov/NCHS/Telemedicine-Use-in-the- } \\
\text { Last-4-Weeks/h7xa-837u [350] }\end{array}$ & $\begin{array}{l}{[350][35} \\
1]\end{array}$ \\
\hline $\begin{array}{l}\text { Center for Disease Control and } \\
\text { Prevention }\end{array}$ & $\begin{array}{l}\text { Provisional COVID-19 } \\
\text { Death Counts in the } \\
\text { United States by County }\end{array}$ & $\begin{array}{l}\text { For analysis and } \\
\text { interpretation }\end{array}$ & $\begin{array}{l}\text { https://data.cdc.gov/NCHS/Provisional-COVID-19- } \\
\text { Death-Counts-in-the-United-St/kn79-hsxy [352] }\end{array}$ & [352] \\
\hline $\begin{array}{l}\text { Center for Disease Control and } \\
\text { Prevention }\end{array}$ & $\begin{array}{l}\text { Provisional COVID-19 } \\
\text { Deaths: Focus on Ages 0- } \\
18 \text { Years }\end{array}$ & $\begin{array}{l}\text { For analysis and } \\
\text { interpretation }\end{array}$ & $\begin{array}{l}\text { https://data.cdc.gov/NCHS/Provisional-COVID-19- } \\
\text { Deaths-Focus-on-Ages-0-18-Yea/nr4s-juj3 [353] }\end{array}$ & [353] \\
\hline $\begin{array}{l}\text { Center for Disease Control and } \\
\text { Prevention }\end{array}$ & $\begin{array}{l}\text { COVID-19 Vaccination } \\
\text { and Case Trends by Age } \\
\text { Group, United States }\end{array}$ & $\begin{array}{l}\text { For analysis and } \\
\text { interpretation }\end{array}$ & $\begin{array}{l}\text { https://data.cdc.gov/Vaccinations/COVID-19- } \\
\text { Vaccination-and-Case-Trends-by-Age-Group-/gxj9-t96f. } \\
\text { [354] }\end{array}$ & [354] \\
\hline $\begin{array}{l}\text { Center for Disease Control and } \\
\text { Prevention }\end{array}$ & $\begin{array}{l}\text { Excess Deaths Associated } \\
\text { with COVID-19 }\end{array}$ & $\begin{array}{l}\text { For analysis and } \\
\text { interpretation }\end{array}$ & $\begin{array}{l}\text { https://data.cdc.gov/NCHS/Excess-Deaths-Associated- } \\
\text { with-COVID-19/xkkf-xrst [355] }\end{array}$ & [355] \\
\hline $\begin{array}{l}\text { Center for Disease Control and } \\
\text { Prevention }\end{array}$ & $\begin{array}{l}\text { Indicators of Health } \\
\text { Insurance Coverage at the } \\
\text { Time of Interview }\end{array}$ & $\begin{array}{l}\text { For analysis and } \\
\text { interpretation }\end{array}$ & $\begin{array}{l}\text { https://data.cdc.gov/NCHS/Indicators-of-Health- } \\
\text { Insurance-Coverage-at-the-Tim/jb9g-gnvr. [356] }\end{array}$ & $\begin{array}{l}{[356][35} \\
7]\end{array}$ \\
\hline $\begin{array}{l}\text { Center for Disease Control and } \\
\text { Prevention }\end{array}$ & $\begin{array}{l}\text { Provisional COVID-19 } \\
\text { Death Counts by Week } \\
\text { Ending Date and State }\end{array}$ & $\begin{array}{l}\text { For analysis and } \\
\text { interpretation }\end{array}$ & $\begin{array}{l}\text { https://data.cdc.gov/NCHS/Provisional-COVID-19- } \\
\text { Death-Counts-by-Week-Ending-D/r8kw-7aab [359] }\end{array}$ & $\begin{array}{l}{[358][35} \\
9]\end{array}$ \\
\hline $\begin{array}{l}\text { Center for Disease Control and } \\
\text { Prevention }\end{array}$ & $\begin{array}{l}\text { COVID-19 Vaccination } \\
\text { Demographics in the } \\
\text { United States, National } \\
\text { data }\end{array}$ & $\begin{array}{l}\text { For analysis and } \\
\text { interpretation }\end{array}$ & $\begin{array}{l}\text { https://data.cdc.gov/Vaccinations/COVID-19- } \\
\text { Vaccination-Demographics-in-the-United-St/km4m-vcsb } \\
\text { [360] }\end{array}$ & [360] \\
\hline $\begin{array}{l}\text { Center for Disease Control and } \\
\text { Prevention }\end{array}$ & $\begin{array}{l}\text { Nationwide Survey on } \\
\text { Commercial Laboratory } \\
\text { Seroprevalence }\end{array}$ & $\begin{array}{l}\text { For analysis and } \\
\text { interpretation }\end{array}$ & $\begin{array}{l}\text { https://data.cdc.gov/Laboratory-Surveillance/Nationwide- } \\
\text { Commercial-Laboratory-Seroprevalence-Su/d2tw-32xv } \\
\text { [361] }\end{array}$ & [361] \\
\hline $\begin{array}{l}\text { Center for Disease Control and } \\
\text { Prevention }\end{array}$ & $\begin{array}{l}\text { Survey on COVID-19 } \\
\text { Hospital Data from the } \\
\text { National Hospital Care }\end{array}$ & $\begin{array}{l}\text { For analysis and } \\
\text { interpretation }\end{array}$ & $\begin{array}{l}\text { https://data.cdc.gov/NCHS/COVID-19-Hospital-Data- } \\
\text { from-the-National-Hospital-/q3t8-zr7t [362] }\end{array}$ & $\begin{array}{l}{[362][36} \\
3]\end{array}$ \\
\hline $\begin{array}{l}\text { Center for Disease Control and } \\
\text { Prevention }\end{array}$ & $\begin{array}{l}\text { Provisional COVID-19 } \\
\text { Death Counts by Age in } \\
\text { Years, 2020-2021 }\end{array}$ & $\begin{array}{l}\text { For analysis and } \\
\text { interpretation }\end{array}$ & $\begin{array}{l}\text { https://data.cdc.gov/NCHS/Provisional-COVID-19- } \\
\text { Death-Counts-by-Age-in-Years-/3apk-4u4f [364] }\end{array}$ & [364] \\
\hline $\begin{array}{l}\text { Center for Disease Control and } \\
\text { Prevention }\end{array}$ & $\begin{array}{l}\text { Long-term Care and } \\
\text { COVID-19 }\end{array}$ & $\begin{array}{l}\text { For analysis and } \\
\text { interpretation }\end{array}$ & $\begin{array}{l}\text { https://data.cdc.gov/NCHS/Long-term-Care-and-COVID- } \\
\text { 19/3j26-kg6d [365] }\end{array}$ & [365] \\
\hline $\begin{array}{l}\text { Center for Disease Control and } \\
\text { Prevention }\end{array}$ & $\begin{array}{l}\text { Provisional COVID-19 } \\
\text { Deaths by Place of Death } \\
\text { and State }\end{array}$ & $\begin{array}{l}\text { For analysis and } \\
\text { interpretation }\end{array}$ & $\begin{array}{l}\text { https://data.cdc.gov/NCHS/Provisional-COVID-19- } \\
\text { Deaths-by-Place-of-Death-and-/uggs-hy5q. [366] }\end{array}$ & [366] \\
\hline $\begin{array}{l}\text { Center for Disease Control and } \\
\text { Prevention }\end{array}$ & $\begin{array}{l}\text { Provisional COVID-19 } \\
\text { Deaths by Week and } \\
\text { Urbanicity }\end{array}$ & $\begin{array}{l}\text { For analysis and } \\
\text { interpretation }\end{array}$ & $\begin{array}{l}\text { https://data.cdc.gov/NCHS/Provisional-COVID-19- } \\
\text { Deaths-by-Week-and-Urbanicity/hkhc-f7hg. [367] }\end{array}$ & $\begin{array}{l}{[367][36} \\
8]\end{array}$ \\
\hline $\begin{array}{l}\text { Center for Disease Control and } \\
\text { Prevention }\end{array}$ & $\begin{array}{l}\text { U.S. State and Territorial } \\
\text { Stay-At-Home Orders: } \\
\text { March 15, } 2020 \text { - August } \\
\text { 15, } 2021 \text { by County by } \\
\text { Day }\end{array}$ & $\begin{array}{l}\text { For analysis and } \\
\text { interpretation }\end{array}$ & $\begin{array}{l}\text { https://data.cdc.gov/Policy-Surveillance/U-S-State-and- } \\
\text { Territorial-Stay-At-Home-Orders-Marc/y2iy-8irm. [369] }\end{array}$ & [369] \\
\hline $\begin{array}{l}\text { Center for Disease Control and } \\
\text { Prevention }\end{array}$ & $\begin{array}{l}\text { U.S. State and Territorial } \\
\text { Public Mask Mandates } \\
\text { from April 10, } 2020 \\
\text { through August 15, } 2021 \\
\text { by County by Day }\end{array}$ & $\begin{array}{l}\text { For analysis and } \\
\text { interpretation }\end{array}$ & $\begin{array}{l}\text { https://data.cdc.gov/Policy-Surveillance/U-S-State-and- } \\
\text { Territorial-Public-Mask-Mandates-Fro/62d6-pm5i [370] }\end{array}$ & $\begin{array}{l}{[370][37} \\
1]\end{array}$ \\
\hline
\end{tabular}




\begin{tabular}{|c|c|c|c|c|}
\hline $\begin{array}{l}\text { Center for Disease Control and } \\
\text { Prevention }\end{array}$ & $\begin{array}{l}\text { U.S. State, Territorial, and } \\
\text { County Stay-At-Home } \\
\text { Orders: March 15-May } 5 \\
\text { by County by Day }\end{array}$ & $\begin{array}{l}\text { For analysis and } \\
\text { interpretation }\end{array}$ & $\begin{array}{l}\text { https://data.cdc.gov/Policy-Surveillance/U-S-State- } \\
\text { Territorial-and-County-Stay-At-Home-Orde/qz3x-mf9n. } \\
\text { [372] }\end{array}$ & {$[372]$} \\
\hline NCHS & $\begin{array}{l}\text { Provisional Death Counts } \\
\text { for Influenza, Pneumonia, } \\
\text { and COVID-19 }\end{array}$ & $\begin{array}{l}\text { For analysis and } \\
\text { interpretation }\end{array}$ & $\begin{array}{l}\text { https://data.cdc.gov/NCHS/Provisional-Death-Counts- } \\
\text { for-Influenza-Pneumonia-a/ynw2-4viq. [373] }\end{array}$ & $\begin{array}{l}{[373][37} \\
4]\end{array}$ \\
\hline $\begin{array}{l}\text { European COVID-19 data } \\
\text { platform }\end{array}$ & $\begin{array}{l}\text { Three data hubs reporting } \\
\text { SARS-CoV-2, COVID-19, } \\
\text { and Federated European } \\
\text { Genome-phenome }\end{array}$ & $\begin{array}{l}\text { For data exploration, } \\
\text { analysis, and } \\
\text { interpretation. }\end{array}$ & $\begin{array}{l}\text { https://www.covid19dataportal.org/the-european- } \\
\text { COVID-19-data-platform [375] }\end{array}$ & [375] \\
\hline Open Safely & $\begin{array}{l}\text { Computational resources } \\
\text { and open access data to } \\
\text { address COVID-19 }\end{array}$ & $\begin{array}{l}\text { For data exploration, } \\
\text { analysis, and } \\
\text { interpretation. }\end{array}$ & $\begin{array}{l}\text { https://datascience.nih.gov/COVID-19-open-access- } \\
\text { resources [376] }\end{array}$ & [376] \\
\hline ImmPort Shared Data & $\begin{array}{l}\text { Research data available to } \\
\text { the public and mostly } \\
\text { scientific community to } \\
\text { improve research work } \\
\text { around COVID-19 }\end{array}$ & $\begin{array}{l}\text { For data exploration, } \\
\text { analysis, and } \\
\text { interpretation. }\end{array}$ & $\begin{array}{l}\text { https://www.immport.org/shared/search?filters=study_2_ } \\
\text { condition_or_disease.condition_preferred:COVID- } \\
\text { 19\%20-\%20DOID:0080600\&utm_source=COVID- } \\
\text { 19\&utm_medium=banner\&utm_campaign=COVID-19 } \\
\text { [377] }\end{array}$ & [377] \\
\hline World Health Organization & $\begin{array}{l}\text { Global COVID-19 } \\
\text { situation for confirmed } \\
\text { cases. }\end{array}$ & $\begin{array}{l}\text { For data exploration, } \\
\text { analysis, and } \\
\text { interpretation. }\end{array}$ & https://covid19.who.int/. [378] & [378] \\
\hline World meter & $\begin{array}{l}\text { Global COVID-19 cases } \\
\text { including confirmed cases, } \\
\text { deaths, active cases, and } \\
\text { closed cases. }\end{array}$ & $\begin{array}{l}\text { For data exploration, } \\
\text { analysis, and } \\
\text { interpretation. }\end{array}$ & https://www.worldometers.info/coronavirus/ [379] & [379] \\
\hline The World Bank & $\begin{array}{l}\text { COVID-19 household } \\
\text { monitoring dashboard. }\end{array}$ & $\begin{array}{l}\text { For data exploration, } \\
\text { analysis, and } \\
\text { interpretation. }\end{array}$ & $\begin{array}{l}\text { https://www.worldbank.org/en/data/interactive/2020/11/1 } \\
\text { 1/COVID-19-high-frequency-monitoring-dashboard } \\
\text { [380] }\end{array}$ & {$[380]$} \\
\hline The World Bank Group & $\begin{array}{l}\text { COVID- } 19 \text { business pulse } \\
\text { survey dashboard that } \\
\text { contains data on the socio- } \\
\text { economic impacts of } \\
\text { COVID-19 in } 76 \text { selected } \\
\text { countries. }\end{array}$ & $\begin{array}{l}\text { For data exploration, } \\
\text { analysis, and } \\
\text { interpretation. }\end{array}$ & $\begin{array}{l}\text { https://www.worldbank.org/en/data/interactive/2021/01/1 } \\
\text { 9/COVID-19-business-pulse-survey-dashboard. [381] }\end{array}$ & $\begin{array}{l}{[381][38} \\
2]\end{array}$ \\
\hline The World Bank Group & $\begin{array}{l}\text { Guidance to World Bank } \\
\text { Group vendors on } \\
\text { COVID-19. }\end{array}$ & $\begin{array}{l}\text { For data exploration, } \\
\text { analysis, and } \\
\text { interpretation. }\end{array}$ & $\begin{array}{l}\text { https://www.worldbank.org/en/about/corporate- } \\
\text { procurement/announcements/guidance_on_COVID-19 } \\
\text { [383] }\end{array}$ & {$[383]$} \\
\hline The World Bank Group & $\begin{array}{l}\text { Harmonized COVID-19 } \\
\text { household monitoring } \\
\text { survey }\end{array}$ & $\begin{array}{l}\text { For data exploration, } \\
\text { analysis, and } \\
\text { interpretation. }\end{array}$ & $\begin{array}{l}\text { https://datacatalog.worldbank.org/search/dataset/0037769 } \\
\text { /Harmonized-COVID-19-Household-Monitoring- } \\
\text { Surveys. [384] }\end{array}$ & {$[384]$} \\
\hline $\begin{array}{l}\text { Centers for Disease Control and } \\
\text { Prevention }\end{array}$ & $\begin{array}{l}\text { Effectiveness of COVID- } \\
19 \text { vaccines }\end{array}$ & $\begin{array}{l}\text { For data exploration, } \\
\text { analysis, and } \\
\text { interpretation. }\end{array}$ & $\begin{array}{l}\text { https://www.cdc.gov/coronavirus/2019- } \\
\text { ncov/vaccines/effectiveness.html. [385] }\end{array}$ & {$[385]$} \\
\hline $\begin{array}{l}\text { Centers for Disease Control and } \\
\text { Prevention }\end{array}$ & $\begin{array}{l}\text { COVID-19 integrated } \\
\text { country view }\end{array}$ & $\begin{array}{l}\text { For data exploration, } \\
\text { analysis, and } \\
\text { interpretation. }\end{array}$ & $\begin{array}{l}\text { https://covid.cdc.gov/covid-data-tracker/\#county-view. } \\
\text { [386] }\end{array}$ & {$[386]$} \\
\hline $\begin{array}{l}\text { Centers for Disease Control and } \\
\text { Prevention }\end{array}$ & $\begin{array}{l}\text { Forecasting cases and } \\
\text { deaths COVID-19 in the } \\
\text { United Sates }\end{array}$ & $\begin{array}{l}\text { For data exploration, } \\
\text { analysis, and } \\
\text { interpretation. }\end{array}$ & $\begin{array}{l}\text { https://covid.cdc.gov/covid-data- } \\
\text { tracker/\#forecasting_weeklydeaths. [387] }\end{array}$ & {$[387]$} \\
\hline $\begin{array}{l}\text { Centers for Disease Control and } \\
\text { Prevention }\end{array}$ & $\begin{array}{l}\text { COVID-19 vaccinations in } \\
\text { the U.S }\end{array}$ & $\begin{array}{l}\text { For data exploration, } \\
\text { analysis, and } \\
\text { interpretation. }\end{array}$ & $\begin{array}{l}\text { https://covid.cdc.gov/covid-data- } \\
\text { tracker/\#vaccinations_vacc-total-admin-rate-total [388] }\end{array}$ & {$[388]$} \\
\hline $\begin{array}{l}\text { Centers for Disease Control and } \\
\text { Prevention }\end{array}$ & $\begin{array}{l}\text { Country-level } \\
\text { vulnerability index in the } \\
\text { United States }\end{array}$ & $\begin{array}{l}\text { For data exploration, } \\
\text { analysis, and } \\
\text { interpretation. }\end{array}$ & $\begin{array}{l}\text { https://covid.cdc.gov/covid-data-tracker/\#pandemic- } \\
\text { vulnerability-index [389] }\end{array}$ & [389] \\
\hline $\begin{array}{l}\text { Centers for Disease Control and } \\
\text { Prevention }\end{array}$ & $\begin{array}{l}\text { COVID-19 community } \\
\text { profile report }\end{array}$ & $\begin{array}{l}\text { For data exploration, } \\
\text { analysis, and } \\
\text { interpretation. }\end{array}$ & $\begin{array}{l}\text { https://healthdata.gov/Health/COVID-19-Community- } \\
\text { Profile-Report/gqxm-d9w9 [390] }\end{array}$ & {$[390]$} \\
\hline
\end{tabular}




\section{CONCLUSION}

The summary tables (Tables I and II) present the technological resources and datasets used in tackling covid-19. Most of the data collected with COVID-19 related to hospitalizations, vaccinations, government response measures, deaths, confirmed reported cases, as well as restrictions and policies are used in aiding the pandemic. The $\mathrm{R}$ resources have mainly been used to develop Shiny apps and dashboards. Java, Kotlin, and Perl resources have been used in developing Android and iOS applications for contact tracing, disease surveillance, fast diagnostics, and notifying users anonymously if they have had any contact with someone who has been infected with COVID-19 via low-power bluetooth technology [28][31][32][51][52][69][129]. Based on the benefits of utilizing these resources, continued research and application of technological resources are highly recommendable [70].

\section{REFERENCES}

[1] https://www.coronatracker.com/

[2] https://github.com/JohnCoene/coronavirus.git

[3] A. Soetewey, "Top 100 R resources on Novel COVID-19 Coronavirus," Towards Data Science, 12 March 2020. [Online]. Available: https://towardsdatascience.com/top-5-r-resources-on-covid-19coronavirus-1d4c8df6d85f. [Accessed 3 October 2021].

[4] https://chschoenenberger.shinyapps.io/covid19_dashboard/

[5] https://github.com/chschoenenberger/covid19_dashboard

[6] https://nicohahn.shinyapps.io/covid19/

[7] https://github.com/nicoFhahn/covid_shiny

[8] https://alhill.shinyapps.io/COVID19seir/

[9] https://github.com/alsnhll/SEIR_COVID19

[10] https://shubhrampandey.shinyapps.io/coronaVirusViz/

[11] https://github.com/shubhrampandey/coronaVirus-dataViz

[12] https://covid19forecast.science.unimelb.edu.au/

[13] https://github.com/benflips/nCovForecast

[14] https://dash.datascienceplus.com/covid19/

[15] https://github.com/CSSEGISandData/COVID-19

[16] D. Anisa, "Map Visualization of COVID-19 Across the World with R," Data Science, 13 March 2020. [Online]. Available: https://datascienceplus.com/map-visualization-of-covid19-across-world/. [Accessed 3 October 2021].

[17] https://thibautfabacher.shinyapps.io/covid-19/

[18] https://github.com/DrFabach/Corona

[19] https://andrecalerovaldez.shinyapps.io/CovidTimeSeriesTest/

[20] https://github.com/Sumidu/covid19shiny

[21] https://tinu.shinyapps.io/Flatten_the_Curve/

[22] https://github.com/tinu-schneider/Flatten_the_Curve

[23] https://jgassen.shinyapps.io/tidycovid19/

[24] https://statsandr.com/blog/top-r-resources-on-covid-19coronavirus/\#tidycovid19

[25] https://sebastianwolf.shinyapps.io/Corona-Shiny/

[26] https://github.com/zappingseb/coronashiny

[27] https://c2m-africa.shinyapps.io/togo-covid-shiny/

[28] http://modestya.securized.net/covid19prediction/

[29] https://www.nathanchaney.com/

[30] N. Chaney, “Animating U.S. COVID-19 hotspots over time,” NathanChaney, 23 October 2020. [Online]. Available: https://www.nathanchaney.com/2020/10/09/animating-u-s-covid-19hotspots-over-time/. [Accessed 3 October 2021].

[31] https://c2m-africa.shinyapps.io/togo-covid-shiny/
[32] https://c2m-africa.shinyapps.io/togo-covid-shiny/

[33] http://modestya.usc.es:3838/covid19prediction/

[34] https://github.com/arnimpdm/Covid-19-prediction

[35] https://jontheepi.shinyapps.io/hcwcoronavirus/

[36] https://github.com/jontheepi/hcwcoronavirus

[37] https://rpubs.com/JMBodart/Covid19-hosp-be

[38] https://github.com/jmbo1190/Covid19

[39] https://covidminder.idea.rpi.edu/

[40] https://github.com/TheRensselaerIDEA/COVIDMINDER

[41] IDEA, "COVIDMINDER: Revealing the regional disparities in outcomes, determinants, and mediations of the COVID-19 pandemic," RENSSELAER POLYTECHNIC INSTITUTE, 2021. [Online]. Available: https://idea.rpi.edu/research/projects/covidminder. [Accessed 04 October 2021].

[42] https://dataenthusiast.ca/apps/covid_ca/

[43] https://milano-r.github.io/erum2020-covidr-contest/petr-baranovskiycovid-ca-data-explorer.html

[44] https://jamalrogersapp.shinyapps.io/tsforecast/

[45] https://github.com/fsmosca/COVID-19-PH-dataset

[46] https://pharmhax.shinyapps.io/covid-corrector-shiny/

[47] https://github.com/pharmhax/covid19-corrector

[48] K. M. Jagodnik, F. Ray, F. M. Giorgi and A. Lachmann, “Correcting under-reported COVID-19 case numbers: estimating the true scale of the pandemic,” medRxiv., 2020.

[49] https://harpomaxx.shinyapps.io/covid19/

[50] https://github.com/harpomaxx/COVID19

[51] https://trafforddatalab.shinyapps.io/covid-19/

[52] https://github.com/traffordDataLab/covid-19

[53] T. D. Lab, “Covid-19 resources.,” Trafford, 2021. [Online]. Available: https://www.trafforddatalab.io/covid19.html. [Accessed 3 October 2021].

[54] https://trafforddatalab.shinyapps.io/trafford_covid-19/

[55] https://github.com/traffordDataLab/trafford_covid-19

[56] https://covid-2019.live/en/

[57] https://github.com/swsoyee/2019-ncov-japan

[58] http://moduloinfo.ca/wordpress/

[59] https://plugins.trac.wordpress.org/browser/covid-19-statistics-displayer

[60] http://coronamapper.com/

[61] https://github.com/JayWelsh/coronamap

[62] https://petolau.shinyapps.io/coronadash/

[63] https://github.com/PetoLau/CoronaDash

[64] https://guillaumepressiat.shinyapps.io/covidfrance/

[65] https://gist.github.com/GuillaumePressiat/0e3658624e42f763e3e6a67df $92 \mathrm{bc} 6 \mathrm{c} 5$

[66] https://nicovidtracker.org/

[67] https://github.com/YouGov-Data/covid-19-tracker

[68] Ulster University, "Ulster University Covid-19 tracker compares NI and ROI data on,” Ulster University, 2020. [Online]. Available: https://www.ulster.ac.uk/news/2020/june/ulster-university-covid-19tracker-compares-ni-and-roi-data-on-coronavirus-testing,-positive-casesand-deaths. [Accessed 3 october 2021].

[69] https://scienceversuscorona.shinyapps.io/covid-overview/

[70] https://github.com/fdabl/Covid-Overview

[71] https://worldhealthorg.shinyapps.io/covid/

[72] https://github.com/WorldHealthOrganization/app

[73] https://www.covidsim.org/v6.20210915/

[74] https://github.com/mrc-ide/squire

[75] https://ramikrispin.github.io/coronavirus/

[76] https://www.dhis2.org/

[77] https://www.dhis2.org/ 
[78] Mobile

phone https://play.google.com/store/apps/details?id=com.dhis2\&hl=en

[79] https://github.com/dhis2/dhis2-covid19-doc

[80] Centre for Disease Control, "Guide to Global Digital Tools for COVID19 Response,” Centre for Disease Control and Prevention, 23 October 2020. [Online]. Available: https://www.cdc.gov/coronavirus/2019ncov/global-covid-19/compare-digital-tools.html. [Accessed 8 October 2021].

[81] https://sormas.org/

[82] https://github.com/hzi-braunschweig/SORMAS-Project

[83] https://www.who.int/godata

[84] https://github.com/godata-who/godata

[85] https://www.cdc.gov/epiinfo/support/downloads.html

[86] https://github.com/Epi-Info/Epi-Info-Community-Edition

[87] https://getodk.org/software/

[88] https://github.com/getodk/collect

[89] https://www.dimagi.com/covid-19/

[90] https://github.com/dimagi/commcare-hq

[91] https://www.kobotoolbox.org

[92] https://www.kobotoolbox.org

[93] https://github.com/kobotoolbox

[94] https://github.com/SHERLOCKLS/Detection-of-COVID-19-frommedical-images

[95] S. Liang, H. Liu, Y. Gu, X. Guo, L. H. L. Li and L. Tao, "Fast automated detection of COVID-19 from medical images using convolutional neural networks," Communications Biology, vol. 4, no. 1, pp. 1-13, 2021.

[96] https://www.bsg.ox.ac.uk/research/research-projects/covid-19government-response-tracker

[97] https://github.com/OxCGRT/covid-policy-tracker

[98] Blavatnik School of Government, "Covid-19 Government Response Tracker,” Blavatnik School of Government, 2021. [Online]. Available: https://www.bsg.ox.ac.uk/research/research-projects/covid-19government-response-tracker. [Accessed 3 October 2021].

[99] https://github.com/pcm-dpc/COVID-19

[100]https://github.com/worldbank/covid-mobile-data

[101]https://radarcovid.gob.es/

[102]https://radarcovid.gob.es/

[103]https://github.com/RadarCOVID/radar-covid-android

[104]van Dijk et al., "COVID RADAR app: Description and validation of population," PloS one,, 2021.

[105]https://covidsafe.cs.washington.edu/

[106]https://github.com/CovidSafe

[107]https://www.canada.ca/en/public-health/services/diseases/coronavirusdisease-covid-19/covid-alert.html

[108]https://www.canada.ca/en/public-health/services/diseases/coronavirusdisease-covid-19/covid-alert.html

[109]https://github.com/cds-snc/covid-alert-app

[110]S.-A. P, “COVID Alert app cost feds \$20M but results 'did not meet expectations':," Global News, 2021. [Online]. Available: https://globalnews.ca/news/8003920/covid-alert-app-expensiveineffective/. [Accessed 3 October 2021].

[111]https://erouska.cz/

[112]https://github.com/covid19cz/erouska-android

[113]Data Proti Covid , "A joint activity of Czech technology companies and IT enthusiasts focused on helping in the fight against the COVID-19 infection,” 2021. [Online]. Available: https://covid19cz.cz/. [Accessed 3 October 2021].

[114]https://www.arcgis.com/apps/opsdashboard/index.html\#/bda7594740fd4 0299423467b48e9ecf6

[115]http://www.arcgis.com/apps/opsdashboard/index.html\#/85320e2ea5424 dfaaa75ae62e5c06e61

[116]https://github.com/sidbannet/COVID-19_analysis [117]https://www.coronawarn.app/en/

[118]https://github.com/corona-warn-app/cwa-app-android

[119]Coroan-Warn-App, “ Help us improve the Corona-Warn-App,” 2021. [Online]. Available: https://www.coronawarn.app/en/. [Accessed 3 October 2021].

[120]https://www.tracetogether.gov.sg/

[121]https://github.com/OpenTrace-Community

[122]Singapore Government Developer Portal, "TraceTogether Community-driven Contact tracing,” 2021. [Online]. Available: https://www.developer.tech.gov.sg/technologies/digital-solutions-toaddress-covid-19/tracetogether. [Accessed 3 October 2021].

[123]https://www.health.govt.nz/our-work/diseases-and-conditions/covid-19novel-coronavirus/covid-19-resources-and-tools/nz-covid-tracer-app

[124]https://apps.apple.com/nz/app/nz-covid-tracer/id1511667597

[125]https://github.com/minhealthnz/nzcovidtracer-app

[126]Ministry of Health New Zealand, "Open-source release of NZ COVID Tracer,” 2020. [Online]. Available: https://www.health.govt.nz/ourwork/diseases-and-conditions/covid-19-novel-coronavirus/covid-19resources-and-tools/nz-covid-tracer-app/about-nz-covid-tracerapp/open-source-release-nz-covid-tracer. [Accessed 4 October 2021].

[127]https://github.com/dsaidgovsg/vigilantgantry

[128]Singapore Government Developer Portal, "VigilantGantry - Access Control with Artificial Intelligence (AI) and Video Analytics,” 2021. [Online]. Available: https://www.developer.tech.gov.sg/technologies/digital-solutions-toaddress-covid-19/vigilantgantry. [Accessed 4 October 2021].

[129]https://git-scm.com/downloads

[130]https://github.com/sdsna/lancet-covid-19-database

[131] The Lancet , “COVID-19 Resource Centre,” Lancet, 2021. [Online]. Available: https://www.thelancet.com/coronavirus. [Accessed 3 October 2021].

[132]https://art-bd.shinyapps.io/covid19canada/

[133]https://github.com/ccodwg/Covid19Canada

[134]https://github.com/mila-iqia/COVI-ML

[135]https://github.com/ImperialCollegeLondon/covid19model

[136]S. Flaxman, S. Mishra, A. Gandy, H. J. T. Unwin, T. A. Mellan, H. Coupland and S. ... Bhatt, "Estimating the effects of non-pharmaceutical interventions on COVID-19 in Europe," Nature, vol. 584, no. 7820, pp. 257-261., 2020.

[137]https://covidtracking.com/

[138]https://github.com/COVID19Tracking

[139]The Covid-19 Tracking project, "How We Made the COVID Tracking Project,” 2021. [Online]. Available: https://covidtracking.com/. [Accessed 3 October 2021].

[140]https://github.com/FedericoGarza/covidmx

[141]Federico, R., "covidmx: Python API to get information about COVID19 in México.Python package version 0.3.1.,” 2020. [Online]. Available: https://github.com/FedericoGarza/covidmx. [Accessed 3 October 2021].

[142] https://covid19-scenarios.org/

[143]https://github.com/neherlab/covid19_scenarios

[144]https://github.com/nhsx/covid-chest-imaging-database

[145]NHSx, "National COVID-19 Chest Imaging Database (NCCID).," National Health Service, 2021. [Online]. Available: https://www.nhsx.nhs.uk/covid-19-response/data-and-covid-19/nationalcovid-19-chest-imaging-database-nccid/. [Accessed 3 October 2021].

[146]https://www.nhsx.nhs.uk/covid-19-response/nhs-covid-pass-verifierapp/international-covid-pass-verifier-app-user-guide/

[147]https://www.nhsx.nhs.uk/covid-19-response/nhs-covid-pass-verifierapp/international-covid-pass-verifier-app-user-guide/

[148]https://github.com/nhsx/covid-pass-verifier

[149]https://github.com/InstituteforDiseaseModeling/covasim

[150]K. e. al., "Determining the optimal strategy for reopening schools, the impact of test and trace interventions, and the risk of occurrence of a 
second COVID-19 epidemic wave in," The Lancet Child \& Adolescent Health, no. https://doi.org/10.1016/S2352-4642(20)30250-9, 2020.

[151] https://covid-19-raffg.herokuapp.com/

[152] https://github.com/raffg/covid-19

[153]https://github.com/QuKunLab/COVID-19

[154]https://github.com/ncbi-nlp/COVID-19-CT-CXR

[155]Y. Peng, Y. Tang, S. Lee, Y. Zhu, R. M. Summers and Z. Lu, “COVID19-CT-CXR: a freely accessible and weakly labeled chest X-ray and CT image collection on COVID-19 from biomedical literature," IEEE transactions on big data, pp. 2-12, 2020.

[156]https://github.com/covidcaremap/covid19-healthsystemcapacity

[157]https://www.closedloop.ai/covid-19-index

[158]https://github.com/closedloop-ai/cv19index

[159]Closed Loop Team, “Open-Source Data Science to Fight Covid-19," 2021. [Online]. Available: https://www.closedloop.ai/covid-19-index. [Accessed 4 October 2021].

[160]https://github.com/BDI-pathogens/OpenABM-Covid19

[161]https://pythonrepo.com/repo/pallupz-covid-vaccine-booking

[162]https://pythonrepo.com/repo/pallupz-covid-vaccine-booking

[163]Mathieu, E., Ritchie, H., Ortiz-Ospina, E. et al. A global database of COVID-19 vaccinations. Nat Hum Behav (2021)

[164]https://ourworldindata.org/covid-vaccinations.

[165]https://ourworldindata.org/covid-deaths.

[166]https://ourworldindata.org/covid-cases.

[167]https://ourworldindata.org/coronavirus-testing.

[168]https://ourworldindata.org/covid-hospitalizations.

[169]https://ourworldindata.org/mortality-risk-covid.

[170]https://ourworldindata.org/mortality-risk-covid\#citation.

[171]https://ourworldindata.org/excess-mortality-covid.

[172] https://ourworldindata.org/policy-responses-covid.

[173]https://ourworldindata.org/identify-covid-exemplars.

[174]https://data.humdata.org/dataset/novel-coronavirus-2019-ncov-cases.

[175]https://data.humdata.org/dataset/indonesia-covid-19-cases-recoveriesand-deaths-per-province.

[176] https://data.humdata.org/dataset/coronavirus-covid-19-cases-and-deaths.

[177]https://data.humdata.org/dataset/oxford-covid-19-government-responsetracker.

[178]https://data.humdata.org/dataset/covid-19-vaccinations.

[179]https://data.humdata.org/dataset/covid-19-global-travel-restrictions-andairline-information.

[180]https://data.humdata.org/dataset/nyt-covid-19-data.

[181] https://data.humdata.org/dataset/total-covid-19-tests-performed-bycountry.

[182]https://data.humdata.org/dataset/global-school-closures-covid19.

[183]https://data.humdata.org/dataset/fair-covid-dataset.

[184]https://data.humdata.org/dataset/district-wise-quarantine-for-covid-19.

[185]https://data.humdata.org/dataset/covid-19-data-visual-inputs.

[186]https://data.humdata.org/dataset/corona-virus-covid-19-cases-anddeaths-in-venezuela.

[187]https://data.humdata.org/dataset/ocha-global-humanitarian-operationalpresence-who-what-where-3w-portal.

[188]https://data.humdata.org/dataset/acaps-covid19-government-measuresdataset.

[189]https://data.humdata.org

[190]https://data.humdata.org/dataset/europe-covid-19-subnational-cases.

[191]https://data.humdata.org/dataset/philippines-covid-19-response-whodoes-what-where.

[192] https://data.humdata.org/dataset/open_one_time_covid_education_impa ct.

[193]https://data.humdata.org/dataset/google-mobility-report.

[194]https://data.humdata.org/dataset/nigeria_covid19_subnational. [195]https://data.humdata.org/dataset/ecdc-covid-19.

[196]https://data.humdata.org/dataset/immunization-campaigns-impacted.

[197]https://data.humdata.org/dataset/financial-times-excess-mortalityduring-covid-19-pandemic-data.

[198]https://data.humdata.org/dataset/state-of-palestine-coronavirus-covid-19subnational-cases.

[199]https://data.humdata.org/dataset/covid-19-symptom-map.

[200]https://data.humdata.org/dataset/covid-19-vaccine-doses-in-hrpcountries.

[201]https://data.humdata.org/dataset/world-bank-indicators-of-interest-tothe-covid-19-outbreak.

[202]http://globalhealth5050.org/covid19.

[203]https://data.humdata.org/dataset/harmonized-covid-19-householdmonitoring-surveys.

[204]https://data.humdata.org/dataset/covid19_africa_continental_infectionsrecoveries-deaths.

[205]https://data.humdata.org/dataset/government-actions-on-covid-19.

[206]https://data.humdata.org/dataset/preventive-health-survey.

[207]https://data.humdata.org/dataset/cameroon-baseline-assessment-dataiom-dtm.

[208]https://data.humdata.org/dataset/covax-round-6-allocations.

[209]https://data.humdata.org/dataset/burkinafaso_covid19_subnational.

[210]https://data.humdata.org/dataset/2019-novel-coronavirus-cases.

[211]https://data.humdata.org/dataset/afghanistan-covid-19-statistics-perprovince.

[212]https://data.humdata.org/dataset/covid-19-mobility-italy.

[213]https://data.humdata.org/dataset/africa-coronavirus-covid-19subnational-cases.

[214]https://data.humdata.org/dataset/covid-19-twitter-data-geographicdistribution.

[215]https://data.humdata.org/dataset/global-covid-19-secondary-impacts.

[216]https://data.humdata.org/dataset/burkinafaso_covid19_city-level.

[217]https://data.humdata.org/dataset/peru-covid19-mortality-rate-in-lima.

[218]https://data.humdata.org/dataset/covid-19-cases-data-in-moscow.

[219]https://data.humdata.org/dataset/world-global-database-of-public-healthand-social-measures-applied-during-the-covid-19-pandemic

[220]https://data.humdata.org/dataset/covid-19-impacts-africa.

[221]https://data.humdata.org/dataset/iraq-coronavirus-covid-19-subnationalcases.

[222]https://data.humdata.org/dataset/iati-covid19-funding.

[223]https://data.humdata.org/dataset/world-bank-and-gavi-vaccinefinancing.

[224]https://data.humdata.org/dataset/open_one_time_covid_impact.

[225]https://data.humdata.org/dataset/ethiopia-covid19-cases.

[226]https://data.humdata.org/dataset/covid-19-high-frequency-indicators. [100]

[227]https://data.humdata.org/dataset/global-humanitarian-response-plancovid-19-administrative-boundaries-and-population-statistics.

[228]https://data.humdata.org/dataset/inform-covid-19-risk-index-version-0$1-4$.

[229]https://data.humdata.org/dataset/kazakhstan-coronavirus-covid-19subnational-cases.

[230]https://data.humdata.org/dataset/philippines-covid-19-operationalpresence-risk-communication-and-community-engagement-rcce.

[231]https://data.humdata.org/dataset/brazil-epidemiological-and-hospitalindicators-on-covid-19-in-ouro-preto.

[232] https://data.humdata.org/dataset/oman-coronavirus-covid-19subnational-cases.

[233]https://data.humdata.org/dataset/mauritania-coronavirus-covid-19-citylevel.

[234]https://data.humdata.org/dataset/rapid-situation-tracking-for-covid-19socioeconomic-impacts. 
[235]https://data.humdata.org/dataset/africa-covid-19-recovered-cases.

[236]https://data.humdata.org/dataset/covid19-impacts-and-vaccineacceptance-in-sub-saharan-africa.

[237]https://data.humdata.org/dataset/un-collective-results-on-the-covid-19socioeconomic-response-in-2020.

[238]https://data.humdata.org/dataset/economic-impact-of-covid-19-in-subsaharan-africa.

[239]https://data.humdata.org/dataset/myanmar-coronavirus-covid-19subnational-cases.

[240]https://data.humdata.org/dataset/ghana-coronavirus-covid-19subnational-cases

[241]https://data.humdata.org/dataset/aid-security-and-covid-19.

[242]https://data.humdata.org/dataset/ngos-affected-by-covid19-russia.

[243]https://data.humdata.org/

[244]https://data.humdata.org/dataset/covax-facility-interim-distributionforecast.

[245]https://data.humdata.org/dataset/unhcr-ken-2020-socioeconomic-impactof-covid-19-on-pocs-in-kenya-v2-2.

[246]https://data.humdata.org/dataset/togo-coronavirus-covid-19-city-level.

[247]https://data.humdata.org/dataset/unicef-indicators-of-interest-to-thecovid-19-outbreak.

[248]https://data.humdata.org/dataset/mozambique-coronavirus-covid-19subnational-cases.

[249]https://data.humdata.org/dataset/haiti-covid-19-subnational-cases

[250]https://data.humdata.org/dataset/constraints-faced-by-people-due-tocovid-19-outbreak.

[251]https://data.humdata.org/dataset/philippines-2020-significant-events.

[252] https://data.humdata.org/dataset/unhcr-ken-2020-covid-round5-v2-1.

[253]https://data.humdata.org/dataset/

[254]https://data.humdata.org/dataset/sudan-covid-19-preparedness-andresponse-4w.

[255]https://data.humdata.org/dataset/community-feedback-by-indonesianred-cross-pmi.

[256]https://data.humdata.org/dataset/sudan-projected-covid-19-sub-nationalcases.

[257]https://data.humdata.org/dataset/travel-restriction-monitoring-iata-covid19-iom-dtm.

[258]https://data.humdata.org/dataset/west-and-central-africa-coronaviruscovid-19-situation.

[259]https://data.humdata.org/dataset/somalia-projected-covid-19-subnational-cases.

[260]https://data.humdata.org/dataset/ethiopia-coronavirus-covid-19subnational-cases.

[261]https://data.humdata.org/dataset/covid-19-cases-data-in-russia.

[262] https://data.humdata.org/dataset/ongoing-impacts-of-covid-19-in-subsaharan-africa.

[263]https://data.humdata.org/dataset/covid-19-global-appeals-and-plans.

[264]https://data.humdata.org/dataset/coronavirus-in-sub-saharan-africa

[265]https://data.humdata.org/dataset/cerf-covid-19-allocations.

[266]https://data.humdata.org/dataset/inform-covid-analysis-v01.

[267]https://data.humdata.org/dataset/covid19-humanitarian-access.

[268]https://data.humdata.org/dataset/lshtm-covid-19-projections.

[269]https://data.humdata.org/dataset/eastern-and-southern-africa-covid-19unicef-situation-and-response.

[270]https://data.humdata.org/dataset/mali-coronavirus-covid-19-subnationalcases.

[271]https://data.humdata.org/dataset/covid-19-economic-exposure-index.

[272]https://data.humdata.org/dataset/somalia-coronavirus-covid-19subnational-cases.

[273]https://data.humdata.org/

[274]https://data.humdata.org/ [275]https://data.humdata.org/dataset/afghanistan-projected-covid-19-subnational-cases.

[276]https://data.humdata.org/dataset/unhcr-bgd-2020-covid-mwtk-v2-1.

[277]https://data.humdata.org/dataset/covid19_risk_index-zip.

[278]https://data.humdata.org/dataset/democratic-republic-of-the-congocoronavirus-covid-19-subnational-cases.

[279]https://data.humdata.org/dataset/libya-coronavirus-covid-19subnational-cases.

[280]https://data.humdata.org/dataset/unhcr-ken-2020-socioeconomic-impactof-covid-19-on-pocs-in-kenya-round2-v1-0.

[281]https://data.humdata.org/dataset/cameroon-covid-19-mobilityrestriction-point-of-entries-iom-dtm.

[282] https://data.humdata.org/dataset/unhcr-ken-2020-covid-panel-v2-1.

[283]https://data.humdata.org/dataset/iraq-projected-covid-19-sub-nationalcases.

[284]https://data.humdata.org/dataset/unhcr-nga-2020-sea-covid19-v2-1.

[285]https://data.humdata.org/

[286]https://data.humdata.org/dataset/ddi-zam-unhcr-covid19-impactassessment-on-refugee-livelihoods-zambia-july-2020.

[287]https://data.humdata.org/dataset/symptomatology-ecu911-santa-cruzmonthly-2018-2021.

[288]https://data.humdata.org/

[289]https://data.humdata.org/dataset/afghanistan-covid-19-health-facilitiesby-province.

[290]https://data.humdata.org/dataset/acaps-covid-19-humanitarianexemptions-dataset

[291]https://data.humdata.org/dataset/south-sudan-covid-19-mobility-andpreparedness-updates-iom-dtm.

[292] https://data.humdata.org/dataset/unhcr-ken-2020-covid-round1-v2-2.

[293]https://data.humdata.org/dataset/unhcr-ken-2020-covid-round4-v2-1.

[294]https://data.humdata.org/dataset/unhcr-ken-2020-covid-round3-v2-1.

[295]https://www.ecdc.europa.eu/en/publications-data/data-covid-19vaccination-eu-eea.

[296]https://www.ecdc.europa.eu/en/publications-data/data-daily-new-casesCOVID-19-eueea-country.

[297]https://www.ecdc.europa.eu/en/publications-data/data-virus-variantsCOVID-19-eueea.

[298]https://www.ecdc.europa.eu/en/publications-data/data-national-14-daynotification-rate-COVID-19.

[299]https://www.ecdc.europa.eu/

[300]https://www.ecdc.europa.eu/en/publications-data/subnational-14-daynotification-rate-COVID-19.

[301] https://www.ecdc.europa.eu/

[302] https://www.ecdc.europa.eu/en/publications-data/download-datahospital-and-icu-admission-rates-and-current-occupancy-COVID-19.

[303]https://www.ecdc.europa.eu/

[304]https://www.ecdc.europa.eu/en/publications-data/download-dataresponse-measures-COVID-19

[305]https://www.ecdc.europa.eu/en/publications-data/COVID-19-data-14day-age-notification-rate-new-cases.

[306]https://www.ecdc.europa.eu/en/publications-data/indicators-mapssupport-council-recommendation.

[307]https://www.ecdc.europa.eu/en/publications-data/download-todays-datageographic-distribution-COVID-19-cases-worldwide.

[308]https://www.kaggle.com/sudalairajkumar/novel-corona-virus-2019dataset

[309]https://www.who.int/emergencies/diseases/novel-coronavirus2019/phsm.

[310]https://ictr.johnshopkins.edu/coronavirus/jh-crown/

[311]https://pcornet.org/news/pcornet-COVID-19-common-data-modellaunched-enabling-rapid-capture-of-insights/

[312]https://covidcp.org/.

[313]https://ncats.nih.gov/n3c/about. 
[314]https://covidclinical.net/plots/paper-01/release-2020-0411/dailycounts.html.

[315]https://covidclinical.net/plots/paper-01/release-2020-0411/demographics.html

[316]https://covidclinical.net/plots/paper-01/release-2020-04-11/labs.html

[317]https://covidclinical.net/plots/paper-01/release-2020-04-11/change.html.

[318]https://covidclinical.net/plots/paper-01/release-2020-04-11/sites.html

[319]https://figshare.com/articles/dataset/Daily_Count_Data_for_Internationa 1_Electronic_Health_Record-Derived_COVID-

19_Clinical_Course_Profile_The_4CE_Consortium/12152976/1.

[320]https://figshare.com/articles/dataset/Demographics_Data_for_Internatio nal_Electronic_Health_Record-Derived_COVID-

19_Clinical_Course_Profile_The_4CE_Consortium/12152973/1

[321]https://figshare.com/articles/dataset/Diagnosis_Data_for_International_ Electronic_Health_Record-Derived_COVID19_Clinical_Course_Profile_The_4CE_Consortium/12152967

[322] https://figshare.com/articles/dataset/Labs_Data_for_International_Electr onic_Health_Record-Derived_COVID-

19_Clinical_Course_Profile_The_4CE_Consortium/12152766

[323]https://figshare.com/articles/dataset/Healthcare_Systems/12118911

[324] https://github.com/CSSEGISandData/COVID19/blob/dcd4181613f512a 6f75249fc77b63286aebe7271/csse_covid_19_data/csse_covid_19_time_ series/time_series_covid19_confirmed_global.csv

[325]https://hrsdata.isr.umich.edu/data-products/2020-hrs-COVID-19-project.

[326] https://covid19researchdatabase.org/.

[327]https://hrs.isr.umich.edu/data-products/COVID-19

[328]https://data.cdc.gov/Case-Surveillance/COVID-19-Case-SurveillancePublic-Use-Data-with-Ge/n8mc-b4w4.

[329]https://data.cdc.gov/Case-Surveillance/COVID-19-Case-SurveillancePublic-Use-Data/vbim-akqf.

[330]https://data.cdc.gov/Case-Surveillance/COVID-19-Case-SurveillanceRestricted-Access-Detai/mbd7-r32t.

[331]https://data.cdc.gov/Vaccinations/COVID-19-Vaccine-DistributionAllocations-by-Juris/w9zu-fywh

[332] https://data.cdc.gov/Vaccinations/COVID-19-Vaccine-DistributionAllocations-by-Juris/saz5-9hgg

[333][205] https://data.cdc.gov/

[334]https://data.cdc.gov/Case-Surveillance/United-States-COVID-19-Casesand-Deaths-by-State-o/9mfq-cb36

[335]https://data.cdc.gov/Vaccinations/COVID-19-Vaccine-DistributionAllocations-by-Juris/b7pe-5nws.

[336]https://data.cdc.gov/

[337]https://data.cdc.gov/Administrative/Provider-Relief-Fund-COVID-19Nursing-Home-Quality/bfqg-cb6d

[338]https://data.cdc.gov/NCHS/Indicators-of-Anxiety-or-Depression-Basedon-Repor/8pt5-q6wp

[339]https://data.cdc.gov/NCHS/Mental-Health-Care-in-the-Last-4Weeks/yni7-er2q

[340]https://data.cdc.gov/

[341]https://data.cdc.gov/Vaccinations/Vaccine-Hesitancy-for-COVID-19County-and-local-es/q9mh-h2tw.

[342] https://data.cdc.gov/NCHS/Loss-of-Work-Due-to-Illness-from-COVID19/qgkx-mswu.

[343]https://data.cdc.gov/Vaccinations/COVID-19-Vaccinations-in-theUnited-States-Jurisdi/unsk-b7fc.

[344]https://data.cdc.gov/Administrative/Provider-Relief-Fund-Acceleratedand-Advance-Payme/v2pi-w3up

[345]https://data.cdc.gov/NCHS/Indicators-of-Reduced-Access-to-Care-Dueto-the-Co/xb3p-q62w.

[346]https://data.cdc.gov/NCHS/Access-and-Use-of-Telemedicine-DuringCOVID-19/8xy9-ubqz.

[347]https://data.cdc.gov/

[348]https://data.cdc.gov/Vaccinations/COVID-19-Vaccination-Trends-inthe-United-States-N/rh2h-3yt2
[349]https://data.cdc.gov/NCHS/Reduced-Access-to-Care-During-COVID19/th9n-ghnr.

[350]https://data.cdc.gov/NCHS/Telemedicine-Use-in-the-Last-4Weeks/h7xa-837u

[351] https://data.cdc.gov/

[352] https://data.cdc.gov/NCHS/Provisional-COVID-19-Death-Counts-inthe-United-St/kn79-hsxy

[353]https://data.cdc.gov/NCHS/Provisional-COVID-19-Deaths-Focus-onAges-0-18-Yea/nr4s-juj3

[354] https://data.cdc.gov/Vaccinations/COVID-19-Vaccination-and-CaseTrends-by-Age-Group-/gxj9-t96f.

[355]https://data.cdc.gov/NCHS/Excess-Deaths-Associated-with-COVID19/xkkf-xrst

[356]https://data.cdc.gov/NCHS/Indicators-of-Health-Insurance-Coverage-atthe-Tim/jb9g-gnvr.

[357]https://data.cdc.gov

[358]https://data.cdc.gov

[359]https://data.cdc.gov/NCHS/Provisional-COVID-19-Death-Counts-byWeek-Ending-D/r8kw-7aab

[360]https://data.cdc.gov/Vaccinations/COVID-19-VaccinationDemographics-in-the-United-St/km4m-vcsb

[361]https://data.cdc.gov/Laboratory-Surveillance/Nationwide-CommercialLaboratory-Seroprevalence-Su/d2tw-32xv

[362] https://data.cdc.gov/NCHS/COVID-19-Hospital-Data-from-theNational-Hospital-/q3t8-zr7t

[363]https://data.cdc.gov/

[364] https://data.cdc.gov/NCHS/Provisional-COVID-19-Death-Counts-byAge-in-Years-/3apk-4u4f

[365]https://data.cdc.gov/NCHS/Long-term-Care-and-COVID-19/3j26-kg6d

[366]https://data.cdc.gov/NCHS/Provisional-COVID-19-Deaths-by-Place-ofDeath-and-/uggs-hy5q.

[367]https://data.cdc.gov/NCHS/Provisional-COVID-19-Deaths-by-Weekand-Urbanicity/hkhc-f7hg.

[368]https://data.cdc.gov/

[369]https://data.cdc.gov/Policy-Surveillance/U-S-State-and-Territorial-StayAt-Home-Orders-Marc/y2iy-8irm.

[370]https://data.cdc.gov/Policy-Surveillance/U-S-State-and-TerritorialPublic-Mask-Mandates-Fro/62d6-pm5i.

[371]https://data.cdc.gov/

[372]https://data.cdc.gov/Policy-Surveillance/U-S-State-Territorial-andCounty-Stay-At-Home-Orde/qz3x-mf9n.

[373]https://data.cdc.gov/NCHS/Provisional-Death-Counts-for-InfluenzaPneumonia-a/ynw2-4viq.

[374]https://data.cdc.gov/NCHS/

[375]https://www.covid19dataportal.org/the-european-COVID-19-dataplatform.

[376]https://datascience.nih.gov/COVID-19-open-access-resources.

[377]https://www.immport.org/shared/search?filters=study_2_condition_or_d isease.condition_preferred:COVID-19\%20-

\%20DOID:0080600\&utm_source=COVID-

19\&utm_medium=banner\&utm_campaign=COVID-19.

[378]https://covid19.who.int/.

[379] https://www.worldometers.info/coronavirus/.

[380]https://www.worldbank.org/en/data/interactive/2020/11/11/COVID-19high-frequency-monitoring-dashboard.

[381]https://www.worldbank.org/en/data/interactive/2021/01/19/COVID-19business-pulse-survey-dashboard.

[382] https://www.worldbank.org.

[383]https://www.worldbank.org/en/about/corporateprocurement/announcements/guidance_on_COVID-19.

[384]https://datacatalog.worldbank.org/search/dataset/0037769/HarmonizedCOVID-19-Household-Monitoring-Surveys.

[385]https://www.cdc.gov/coronavirus/2019ncov/vaccines/effectiveness.html. 
[386]https://covid.cdc.gov/covid-data-tracker/\#county-view.

[387]https://covid.cdc.gov/covid-data-tracker/\#forecasting_weeklydeaths.

[388]https://covid.cdc.gov/covid-data-tracker/\#vaccinations_vacc-totaladmin-rate-total.

[389]https://covid.cdc.gov/covid-data-tracker/\#pandemic-vulnerability-index.

[390]https://healthdata.gov/Health/COVID-19-Community-ProfileReport/gqxm-d9w9.

[391]Muniswamaiah, Manoj, Tilak Agerwala, and Charles C. Tappert. "Survey of the use of digital technologies to combat COVID-19." In 2020 IEEE International Conference on Big Data (Big Data), pp. 57685771. IEEE, 2020.
[392]Hannah Ritchie, Edouard Mathieu, Lucas Rodés-Guirao, Cameron Appel, Charlie Giattino, Esteban Ortiz-Ospina, Joe Hasell, Bobbie Macdonald, Diana Beltekian and Max Roser (2020) - "Coronavirus Pandemic (COVID-19)". Published online at OurWorldInData.org. Retrieved from: 'https://ourworldindata.org/coronavirus' (Online Resource).

[393]Hasell, J., Mathieu, E., Beltekian, D. et al. A cross-country database of COVID-19 testing. Sci Data 7, 345 (2020) 